\title{
INTRINSIC LIPSCHITZ GRAPHS IN CARNOT GROUPS OF STEP 2
}

\author{
Daniela Di Donato \\ Università di Trento, Dipartimento di Matematica \\ Via Sommarive 14, 38123, Povo (Trento), Italy; daniela.didonato@unitn.it
}

\begin{abstract}
We provide a characterization of locally intrinsic Lipschitz functions in Carnot groups of step 2 in terms of their intrinsic distributional gradients. We also prove an area formula with respect to the spherical Hausdorff measure for the graph of an intrinsic Lipschitz function.
\end{abstract}

\section{Introduction}

The notion of rectifiable set is a key one in calculus of variations and in geometric measure theory. To develop a satisfactory theory of rectifiable sets inside Carnot groups has been the object of much research in the last years. For a general theory of rectifiable sets in euclidean spaces one can see $[13,14,32]$ while a general theory in metric spaces can be found in [2].

Rectifiable sets are classically defined as contained in the countable union of $\mathbf{C}^{1}$ submanifolds. An equivalent characterization can be derived from a Rademacher type theorem, which is valid in a special subclass of Carnot groups including groups of step 2 (see Theorem 4.3.5 in [16]). As in Euclidean spaces, 1-codimensional rectifiable sets in these groups can be equivalently defined using intrinsic $\mathbf{C}^{1}$ submanifolds or intrinsic Lipschitz graphs (see Proposition 4.4.4 in [16]). Hence, understanding these objects, which naturally take the role of $\mathbf{C}^{1}$ submanifolds or Lipschitz graphs, is a preliminary task in developing a satisfactory theory of rectifiable sets inside Carnot groups.

In this paper we focus our attention on the notion of intrinsic Lipschitz graphs inside the Carnot groups. A Carnot group G is a connected, simply connected and stratified Lie group and has a sufficiently rich compatible underlying structure, due to the existence of intrinsic families of left translations and dilations and depending on the horizontal vector fields which generate the horizontal layer. We call intrinsic any notion depending directly by the structure and geometry of G. For a complete description of Carnot groups [7, 29, 37] are recommended.

Euclidean spaces are commutative Carnot groups and are the only commutative ones. The simplest but, at the same time, non-trivial instances of non-Abelian Carnot groups are provided by the Heisenberg groups $\mathbf{H}^{n}$ (see for instance $[7,37]$ ).

We begin by recalling that an intrinsic regular hypersurface (i.e. a topological codimension 1 surface) $S \subset \mathbf{G}$ is locally defined as a non critical level set of a $\mathbf{C}^{1}$ intrinsic function. More precisely, there exists a continuous function $f: \mathbf{G} \rightarrow \mathbf{R}$ such that locally $S=\{p \in \mathbf{G}: f(p)=0\}$ and the intrinsic gradient $\nabla_{\mathbf{G}} f=$

https://doi.org/10.5186/aasfm.2020.4556

2010 Mathematics Subject Classification: Primary 35R03; Secondary 28A75, 53C17.

Key words: Carnot groups, intrinsic Lipschitz functions, intrinsic graphs, intrinsic regular hypersurfaces.

D. D. D. is supported by University of Trento, Italy. 
$\left(X_{1} f, \ldots, X_{m} f\right)$ exists in the sense of distributions and it is continuous and non vanishing on $S$. In a similar way, a $k$-codimensional regular surface $S \subset \mathbf{G}$ is locally defined as a non critical level set of a $\mathbf{C}^{1}$ intrinsic vector function $f=\left(f_{1}, \ldots, f_{k}\right): \mathbf{G} \rightarrow$ $\mathbf{R}^{k}$.

On the other hand, the intrinsic graphs came out naturally in [18], while studying level sets of Pansu differentiable functions from $\mathbf{H}^{n}$ to $\mathbf{R}$. The simple idea of intrinsic graph is the following one: let $\mathbf{M}$ and $\mathbf{W}$ be complementary subgroups of $\mathbf{G}$, i.e. homogeneous subgroups such that $\mathbf{W} \cap \mathbf{M}=\{0\}$ and $\mathbf{G}=\mathbf{W} \cdot \mathbf{M}$ (here indicates the group operation in $\mathbf{G}$ and 0 is the unit element), then the intrinsic left graph of $\phi: \mathbf{W} \rightarrow \mathbf{M}$ is the set

$$
\operatorname{graph}(\phi):=\{a \cdot \phi(a) \mid a \in \mathbf{W}\} .
$$

Hence the existence of intrinsic graphs depends on the possibility of splitting $\mathbf{G}$ as a product of complementary subgroups hence it depends on the structure of the Lie algebra associated to $\mathbf{G}$.

Specifically the notion of intrinsic Lipschitz graphs has been introduced with different degrees of generality in $[4,16,21,18,28]$. In [17] the authors provide a comprehensive presentation of this theory. A function $\phi: \mathcal{O} \subset \mathbf{W} \rightarrow \mathbf{M}$ is said to be intrinsic Lipschitz if it is possible to put, at each point $p \in \operatorname{graph}(\phi)$, an intrinsic cone (see Definition 2.10) with vertex $p$, axis $\mathbf{M}$ and fixed opening, intersecting graph $(\phi)$ only at $p$. We call a set $S \subset \mathbf{G}$ an intrinsic Lipschitz graph if there exists an intrinsic Lipschitz function $\phi: \mathcal{O} \subset \mathbf{W} \rightarrow \mathbf{M}$ such that $S=\operatorname{graph}(\phi)$ for suitable complementary subgroups $\mathbf{W}$ and $\mathbf{M}$ of $\mathbf{G}$.

Through the implicit function theorem (see [19, 30]), it is known that every $k$-codimensional regular surface can be locally represented as intrinsic graph of a function $\phi: \mathcal{O} \subset \mathbf{W} \rightarrow \mathbf{M}$. Conversely, we established in [11] that the intrinsic graph of an uniformly intrinsically differentiable map is a regular surface. A function is intrinsic differentiable if it is well approximated by appropriate linear type functions, denoted intrinsic linear functions (see Section 2). In particular, when $\mathbf{W}$ and $\mathbf{M}$ are both normal subgroup, this notion corresponds to that of Pansu differentiability.

When $\mathbf{M}$ is one dimensional we can identify $\phi: \mathcal{O} \subset \mathbf{W} \rightarrow \mathbf{M}$ with a real valued continuous function defined on a one codimensional homogeneous subgroup of $\mathbf{G}$ (see Remark 3.3). In this case in Heisenberg groups the parameterization $\phi$ is weak solution of a system of non-linear first order PDEs $D^{\phi} \phi=w$, where $w$ is a continuous map and $D^{\phi}$ are suitable 'derivatives' $D_{j}^{\phi} \phi$ of $\phi$ (see $[3,6,40]$ ). The non linear first order differential operators $D_{j}^{\phi}$ were introduced by Serra Cassano et al. in the context of Heisenberg groups $\mathbf{H}^{n}$ (see [37] and the references therein).

Following the notations in [37] the operators $D_{j}^{\phi}$ are called intrinsic derivatives of $\phi$; and $D^{\phi} \phi$, the vector of the intrinsic derivatives of $\phi$, is the intrinsic gradient of $\phi$. In the first Heisenberg group $\mathbf{H}^{1}$ the operator $D^{\phi}$ reduces to the classical Burgers' operator. In a general Carnot group of step $\kappa, D_{j}^{\phi} \phi$ is the projection on $\mathbf{W}$ of a horizontal vector field of $\mathbf{G}$ on the points of the intrinsic graph of $\phi$ (see [28]).

More generally, Kozhenikov talks about projected vector fields and he consider the projection on $\mathbf{W}$ of elements belong to Lie algebra of $\mathbf{W}$ (see Definition 4.2.12, [28]). He characterizes intrinsic Lipschitz graphs and regular surfaces in terms of metric properties of integral curves of these projected vector fields (see Theorem 4.2.16 and Theorem 4.3.1 in [28]).

In $[11,12]$ we give an explicit form of intrinsic derivatives of $\phi: \mathcal{O} \subset \mathbf{W} \rightarrow \mathbf{M}$, where $\mathbf{G}=\mathbf{W} \cdot \mathbf{M}$ is a Carnot group of step 2 (see Section 5.1, [7]) and then we 
extend Theorem 1.3 and Theorem 5.7 in [3] proved in $\mathbf{H}^{n}$. Precisely, we prove that the intrinsic graph of continuous map $\phi$ is a regular hypersurface if and only if $\phi$ and its intrinsic gradient can be uniformly approximated by $\mathbf{C}^{1}$ functions and $\phi$ is distributional solution of PDEs' system $D^{\phi} \phi=w$, with $w$ is a continuous map. Moreover, we also show that these assumptions are equivalent to the fact that $\phi$ is broad* solution of $D^{\phi} \phi=w$ (see Definition 8.1) and it is little 1/2-Hölder continuous (see Proposition $8.1(1)$ ). In $\mathbf{H}^{1}$ the notion of broad* ${ }^{*}$ solution extends the classical notion of broad solution for Burgers' equation through characteristic curves provided $\phi$ and $w$ are locally Lipschitz continuous. In our case $\phi$ and $w$ are supposed to be only continuous then the classical theory breaks down. On the other hand broad* solutions of the system $D^{\phi} \phi=w$ can be constructed with a continuous datum $w$.

The main contribution of this paper is to provide counterparts for results that were previously known in Heisenberg groups in the setting of arbitrary Carnot groups of step 2:

- Theorem 4.1,4.2 generalize a result by Monti and Vittone in [34]

- Theorem 5.5,6.1 generalize results by Citti, Manfredini, Pinamonti, Serra Cassano in [9]

- Theorem 7.1,7.2 generalize a result by Bigolin, Caravenna, Serra Cassano in $[5]$

- Theorem 8.2 generalizes a result by Ambrosio, Serra Cassano, Vittone in [3]

They are often proven by similar arguments. The main difference between 2 step Carnot groups and Heisenberg groups is that in $\mathbf{H}^{n}$ there is only one vertical (i.e. non-horizontal) coordinate, whereas for 2 step Carnot groups there can be many.

Precisely we show that a locally intrinsic Lipschitz map $\phi$ is a continuous distributional solution of the non-linear first order PDEs' system $D^{\phi} \phi=w$, where $w$ is only a measurable function (see Theorem 7.1). This is a direct consequence of the fact that $\phi$ can be approximated by a sequence of smooth maps, with pointwise convergent intrinsic gradient (see Theorem 6.1).

Moreover the opposite implication is true when $\phi$ is also locally $1 / 2$-Hölder continuous map along the vertical components (see Theorem 7.2). In order to establish this statement we need a preliminary result: Lemma 7.3. Here we prove that a locally 1/2-Hölder continuous map along the vertical components, which is also a continuous distributional solution of the system $D^{\phi} \phi=w$, is a Lipschitz map in classical sense along any characteristic curve of the vector fields $D^{\phi}$.

Finally in the last section, we consider the system $D^{\phi} \phi=w$, where $w$ is supposed to be a continuous map and not just measurable function as opposed to the previous sections. We give a characterization of G-regular hypersurfaces in terms of distributional solution of this system. Our strategy will be to prove that each continuous distributional solution of the system $D^{\phi} \phi=w$ is a broad* solution and vice versa (see Proposition 8.1).

The plan of the work is the following:

In Section 2 we provide the definitions and some properties about the differential calculus within Carnot groups. In particular we give the general definitions and some basic tools of intrinsic differentiable and intrinsic Lipschitz maps.

Section 3 is specialized to Carnot groups of step 2 (see Chapter 3, [7]).

Section 4 is dedicated to Caccioppoli sets in Carnot groups of step 2. We show that the boundary of sets with finite G-perimeter and having a bound on the orientation of the measure theoretic normal is the intrinsic graph of an intrinsic Lipschitz function (see Theorem 4.2). 
Sections 5 and 6 contain an area formula with respect to the spherical Hausdorff measure for the graph of an intrinsic Lipschitz function (see Theorem 5.7) and we characterize intrinsic Lipschitz functions as maps which can be approximated by a sequence of smooth maps, with pointwise convergent and uniformly bounded intrinsic gradient (see Theorem 6.1).

Section 7 contains the main results of this paper, i.e. Theorem 7.1 and Theorem 7.2 .

Section 8 is dedicated to G-regular hypersurafces. We show that if we consider a locally little $1 / 2$-Hölder continuous map along the vertical components, then it is a distributional solution of the system $D^{\phi} \phi=w$, where $w$ is a given continuous function if and only if its graph is a G-regular hypersurface (see Theorem 8.2).

Acknowledgements. We wish to express our gratitude to R. Serapioni and F. Serra Cassano, for having signaled us this problem and for many invaluable discussions during our PhD at University of Trento. We thank B. Franchi, A. Pinamonti and D. Vittone for useful discussions and important suggestions on the subject.

\section{Notations and preliminary results}

Carnot groups. We begin by recalling briefly the definition of Carnot groups. For a general account see e.g. [7, 17, 29, 37].

A Carnot group $\mathbf{G}=\left(\mathbf{G}, \cdot, \delta_{\lambda}\right)$ of step $\kappa$ is a connected and simply connected Lie group whose Lie algebra $\mathfrak{g}$ admits a stratification, i.e. a direct sum decomposition $\mathfrak{g}=V_{1} \oplus V_{2} \oplus \cdots \oplus V_{\kappa}$, with the following property: the entire Lie algebra $\mathfrak{g}$ is generated by its first layer $V_{1}$, the so called horizontal layer, that is

$$
\left\{\begin{array}{l}
{\left[V_{1}, V_{l-1}\right]=V_{l} \quad \text { if } 2 \leq l \leq \kappa,} \\
{\left[V_{1}, V_{\kappa}\right]=\{0\} .}
\end{array}\right.
$$

We denote by $N$ the dimension of $\mathfrak{g}$ and by $n_{s}$ the dimension of $V_{s}$.

The exponential map exp: $\mathfrak{g} \rightarrow \mathbf{G}$ is a global diffeomorphism from $\mathfrak{g}$ to $\mathbf{G}$. Hence, if we choose a basis $\left\{X_{1}, \ldots, X_{N}\right\}$ of $\mathfrak{g}$, any $p \in \mathbf{G}$ can be written in a unique way as $p=\exp \left(p_{1} X_{1}+\cdots+p_{N} X_{N}\right)$ and we can identify $p$ with the $N$-tuple $\left(p_{1}, \ldots, p_{N}\right) \in \mathbf{R}^{N}$ and $\mathbf{G}$ with $\left(\mathbf{R}^{N}, \cdot, \delta_{\lambda}\right)$. The identity of $\mathbf{G}$ is the origin of $\mathbf{R}^{N}$.

For any $\lambda>0$, the (non isotropic) dilations $\delta_{\lambda}: \mathbf{G} \rightarrow \mathbf{G}$ are automorphisms of $\mathbf{G}$ and are defined as

$$
\delta_{\lambda}\left(p_{1}, \ldots, p_{N}\right)=\left(\lambda^{\alpha_{1}} p_{1}, \ldots, \lambda^{\alpha_{N}} p_{N}\right)
$$

where $\alpha_{l} \in \mathbf{N}$ is called homogeneity of the variable $p_{l}$ in $\mathbf{G}$ and is given by $\alpha_{l}=j$ whenever $m_{j-1}<l \leq m_{j}$ with $m_{s}-m_{s-1}=n_{s}$. Hence $1=\alpha_{1}=\cdots=\alpha_{m_{1}}<$ $\alpha_{m_{1}+1}=2 \leq \cdots \leq \alpha_{N}=\kappa$.

For any $p \in \mathbf{G}$ the intrinsic left translation $\tau_{p}: \mathbf{G} \rightarrow \mathbf{G}$ are defined as

$$
q \mapsto \tau_{p} q:=p \cdot q=p q
$$

The explicit expression of the group operation - is determined by the CampbellHausdorff formula. It has the form

$$
p \cdot q=p+q+\mathcal{Q}(p, q) \text { for all } p, q \in \mathbf{G} \equiv \mathbf{R}^{N},
$$

where $\mathcal{Q}=\left(\mathcal{Q}_{1}, \ldots, \mathcal{Q}_{N}\right): \mathbf{R}^{N} \times \mathbf{R}^{N} \rightarrow \mathbf{R}^{N}$. Here $\mathcal{Q}_{l}(p, q)=0$ for each $l=$ $1, \ldots, m_{1}$ and each $1<j \leq \kappa$ and $m_{j-1}+1 \leq l \leq m_{j}$ we have $\mathcal{Q}_{l}(p, q)=$ $\mathcal{Q}_{l}\left(\left(p_{1}, \ldots, p_{m_{j-1}}\right),\left(q_{1}, \ldots, q_{m_{j-1}}\right)\right)$. Moreover every $\mathcal{Q}_{l}$ is a homogeneous polynomial of degree $\alpha_{l}$ with respect to the intrinsic dilations of $\mathbf{G}$. It is useful to think 
$\mathbf{G}=\mathbf{G}^{1} \oplus \mathbf{G}^{2} \oplus \cdots \oplus \mathbf{G}^{\kappa}$ where $\mathbf{G}^{l}=\exp \left(V_{l}\right)=\mathbf{R}^{n_{i}}$ is the $l^{\text {th }}$ layer of $\mathbf{G}$ and to write $p \in \mathbf{G}$ as $\left(p^{1}, \ldots, p^{\kappa}\right)$ with $p^{l} \in \mathbf{G}^{l}$. According to this

(1) $p \cdot q=\left(p^{1}+q^{1}, p^{2}+q^{2}+\mathcal{Q}^{2}\left(p^{1}, q^{1}\right), \ldots, p^{\kappa}+q^{\kappa}+\mathcal{Q}^{\kappa}\left(\left(p^{1}, \ldots, p^{\kappa-1}\right),\left(q^{1}, \ldots, q^{\kappa-1}\right)\right)\right.$ for every $p=\left(p^{1}, \ldots, p^{\kappa}\right), q=\left(q^{1}, \ldots, q^{\kappa}\right) \in \mathbf{G}$. By Theorem 3.2 .2 in [7], we know that

$$
p^{-1}=\left(-p^{1}, \ldots,-p^{\kappa}\right)
$$

The norm of $\mathbf{R}^{n_{s}}$ is denoted with the symbol $|\cdot| \mathbf{R}^{n_{s}}$.

An absolutely continuous curve $\gamma:[0, T] \rightarrow \mathbf{G}$ is a sub-unit curve with respect to $X_{1}, \ldots, X_{m_{1}}$ if it is an horizontal curve, that is if there are real measurable functions $h_{1}(t), \ldots, h_{m_{1}}(t), t \in[0, T]$ such that

$$
\dot{\gamma}(s)=\sum_{l=1}^{m_{1}} h_{l}(t) X_{l}(\gamma(t)), \quad \text { for a.e. } t \in[0, T],
$$

and if $\sum_{l=1}^{m_{1}} h_{l}^{2} \leq 1$.

Definition 2.1. (Carnot-Carathéodory distance) If $p, q \in \mathbf{G}$, their cc-distance $d_{c c}(p, q)$ is

$$
d_{c c}(p, q)=\inf \{T>0 \text { : there is a subunit curve } \gamma \text { with } \gamma(0)=p, \gamma(T)=q\}
$$

The set of subunit curves joining $p$ and $q$ is not empty, by Chow's theorem, since the rank of the Lie algebra generated by $X_{1}, \ldots, X_{m_{1}}$ is $N$; hence $d_{c c}$ is a distance on $\mathbf{G}$ inducing the same topology as the standard Euclidean distance.

A homogeneous norm on $\mathbf{G}$ is a nonnegative function $p \mapsto\|p\|$ such that for all $p, q \in \mathbf{G}$ and for all $\lambda \geq 0$

$$
\begin{aligned}
\|p\| & =0 \quad \text { if and only if } p=0 \\
\left\|\delta_{\lambda} p\right\| & =\lambda\|p\|, \quad\|p \cdot q\| \leq\|p\|+\|q\| .
\end{aligned}
$$

There is a particular homogeneous norm defined in Theorem 5.1 in [20], as following

$$
\left\|\left(p^{1}, \ldots, p^{\kappa}\right)\right\|:=\max _{s=1, \ldots, \kappa}\left\{\epsilon_{s}\left|p^{s}\right|_{\mathbf{R}^{n_{s}}}^{1 / s}\right\} \quad \text { for all }\left(p^{1}, \ldots, p^{\kappa}\right) \in \mathbf{G}
$$

with $\epsilon_{1}=1$, and $\epsilon_{s} \in(0,1]$ depending on the structure of the group for $s=2, \ldots, \kappa$. This homogeneous norm is symmetric, i.e. $\|p\|=\left\|p^{-1}\right\|$ for all $p \in \mathbf{G}$ and such that

$$
\left\|\left(p^{1}, 0 \ldots, 0\right)\right\|=\left|p^{1}\right|_{\mathbf{R}^{n_{1}}},
$$

for all $p^{1} \in \mathbf{R}^{n_{1}}$. Given any homogeneous norm $\|\cdot\|$, it is possible to introduce a distance in $\mathbf{G}$ given by

$$
d(p, q)=d\left(p^{-1} q, 0\right)=\left\|p^{-1} q\right\| \quad \text { for all } p, q \in \mathbf{G} .
$$

We observe that any distance $d$ obtained in this way is always equivalent with the Carnot-Carathéodory's distance $d_{c c}$ of the group. Both $d$ and $d_{c c}$ are well behaved with respect to left translations and dilations, i.e. for all $p, q, q^{\prime} \in \mathbf{G}$ and $\lambda>0$,

$$
d\left(p \cdot q, p \cdot q^{\prime}\right)=d\left(q, q^{\prime}\right), \quad d\left(\delta_{\lambda} q, \delta_{\lambda} q^{\prime}\right)=\lambda d\left(q, q^{\prime}\right) .
$$

Moreover, for any bounded subset $\Omega \subset \mathbf{G}$ there exist positive constants $c_{1}=$ $c_{1}(\Omega), c_{2}=c_{2}(\Omega)$ such that for all $p, q \in \Omega$

$$
c_{1}|p-q|_{\mathbf{R}^{N}} \leq d(p, q) \leq c_{2}|p-q|_{\mathbf{R}^{N}}^{1 / \kappa}
$$


and, in particular, the topology induced on $\mathbf{G}$ by $d$ is the Euclidean topology. For $p \in \mathbf{G}$ and $r>0, \mathcal{U}(p, r)$ will be the open ball associated with the distance $d$. Intrinsic $t$-dimensional spherical Hausdorff measure $\mathcal{S}^{t}$ on $\mathbf{G}, t \geq 0$, is obtained from $d$, following Carathéodory construction (see for instance [32]).

W.l.o.g. we consider the homogeneous norm defined in (3). Indeed from the fact that all the homogeneous norms are equivalent, all the estimates we will use hold with an arbitrary homogeneous norm. On the other hand, Theorem 2.4, Theorem 2.5 and Theorem 5.7 are only proven for specific choices of left invariant distance $d_{\infty}$, which is the distance induced by the maximum norm (3). We will emphasize this last fact at the beginning of each statement.

The Hausdorff dimension of $(\mathbf{G}, d)$ as a metric space is denoted homogeneous dimension of $\mathbf{G}$ and it can be proved to be the integer $\mathfrak{q}:=\sum_{j=1}^{N} \alpha_{j}=\sum_{l=1}^{\kappa} i$ $\operatorname{dim} V_{l} \geq N$ (see [33]). The subbundle of the tangent bundle $T \mathbf{G}$, spanned by the vector fields $X_{1}, \ldots, X_{m_{1}}$ plays a particularly important role in the theory, and is called the horizontal bundle $H \mathbf{G}$; the fibers of $H \mathbf{G}$ are

$$
H \mathbf{G}_{p}=\operatorname{span}\left\{X_{1}(p), \ldots, X_{m_{1}}(p)\right\}, \quad p \in \mathbf{G} .
$$

A sub Riemannian structure is defined on $\mathbf{G}$, endowing each fiber of $H \mathbf{G}$ with a scalar product $\langle\cdot, \cdot\rangle_{p}$ and a norm $|\cdot|_{p}$ making the basis $X_{1}(p), \ldots, X_{m_{1}}(p)$ an orthonormal basis. Hence, if $v=\sum_{l=1}^{m_{1}} v_{l} X_{l}(p)=v^{1}$ and $w=\sum_{l=1}^{m_{1}} w_{l} X_{l}(p)=w^{1}$ are in $H \mathbf{G}$, then $\langle v, w\rangle_{p}:=\sum_{l=1}^{m_{1}} v_{l} w_{l}$ and $|v|_{p}^{2}:=\langle v, v\rangle_{p}$. We will write, with abuse of notation, $\langle\cdot, \cdot\rangle$ meaning $\langle\cdot, \cdot\rangle_{p}$ and $|\cdot|$ meaning $|\cdot|_{p}$.

The sections of $H \mathbf{G}$ are called horizontal sections, a vector of $H \mathbf{G}_{p}$ is a horizontal vector while any vector in $T \mathbf{G}_{p}$ that is not horizontal is a vertical vector.

The Haar measure of the group $\mathbf{G}=\mathbf{R}^{N}$ is the Lebesgue measure $d \mathcal{L}^{N}$. It is left (and right) invariant. Various Lebesgue spaces on $\mathbf{G}$ are meant always with respect to the measure $d \mathcal{L}^{N}$ and are denoted as $\mathcal{L}^{p}(\mathbf{G})$.

2.2. $\mathbf{C}_{\mathbf{G}}^{1}$ functions, G-regular surfaces, Caccioppoli sets. (See [37]) In [35] Pansu introduced an appropriate notion of differentiability for functions acting between Carnot groups. We recall this definition in the particular instance that is relevant here.

Let $\mathcal{U}$ be an open subset of a Carnot group $\mathbf{G}$. A function $f: \mathcal{U} \rightarrow \mathbf{R}^{k}$ is Pansu differentiable or more simply P-differentiable in $a \in \mathcal{U}$ if there is a homogeneous homomorphism

$$
d_{\mathbf{P}} f(a): \mathbf{G} \rightarrow \mathbf{R}^{k},
$$

the Pansu differential of $f$ in $a$, such that, for $b \in \mathcal{U}$,

$$
\lim _{r \rightarrow 0^{+}} \sup _{0<\left\|a^{-1} b\right\|<r} \frac{\left|f(b)-f(a)-d_{\mathbf{P}} f(a)\left(a^{-1} b\right)\right|_{\mathbf{R}^{k}}}{\left\|a^{-1} b\right\|}=0 .
$$

Saying that $d_{\mathbf{P}} f(a)$ is a homogeneous homomorphism we mean that $d_{\mathbf{P}} f(a): \mathbf{G} \rightarrow \mathbf{R}^{k}$ is a group homomorphism and also that $d_{\mathbf{P}} f(a)\left(\delta_{\lambda} b\right)=\lambda d_{\mathbf{P}} f(a)(b)$ for all $b \in \mathbf{G}$ and $\lambda \geq 0$.

Observe that, later on in Definition 2.8, we give a different notion of differentiability for functions acting between subgroups of a Carnot group and we reserve the notation $d f$ or $d f(a)$ for that differential.

We denote $\mathbf{C}_{\mathbf{G}}^{1}\left(\mathcal{U}, \mathbf{R}^{k}\right)$ the set of functions $f: \mathcal{U} \rightarrow \mathbf{R}^{k}$ that are P-differentiable in each $a \in \mathcal{U}$ and such that $d_{\mathbf{P}} f(a)$ depends continuously on $a$. It can be proved that $f=\left(f_{1}, \ldots, f_{k}\right) \in \mathbf{C}_{\mathbf{G}}^{1}\left(\mathcal{U}, \mathbf{R}^{k}\right)$ if and only if the distributional horizontal derivatives 
$X_{l} f_{j}$, for $l=1 \ldots, m_{1}, j=1, \ldots, k$, are continuous in $\mathcal{U}$. Remember that $\mathbf{C}^{1}(\mathcal{U}, \mathbf{R}) \subset$ $\mathbf{C}_{\mathbf{G}}^{1}(\mathcal{U}, \mathbf{R})$ with strict inclusion whenever $\mathbf{G}$ is not abelian (see Remark 6 in [18]).

The horizontal Jacobian (or the horizontal gradient if $k=1$ ) of $f: \mathcal{U} \rightarrow \mathbf{R}^{k}$ in $a \in \mathcal{U}$ is the matrix

$$
\nabla_{\mathbf{G}} f(a):=\left[X_{l} f_{j}(a)\right]_{l=1 \ldots m_{1}, j=1 \ldots k}
$$

when the partial derivatives $X_{i} f_{j}$ exist. Hence $f=\left(f_{1}, \ldots, f_{k}\right) \in \mathbf{C}_{\mathbf{G}}^{1}\left(\mathcal{U}, \mathbf{R}^{k}\right)$ if and only if its horizontal Jacobian exists and is continuous in $\mathcal{U}$. The horizontal divergence of $\phi:=\left(\phi_{1}, \ldots, \phi_{m_{1}}\right): \mathcal{U} \rightarrow \mathbf{R}^{m_{1}}$ is defined as

$$
\operatorname{div}_{\mathbf{G}} \phi:=\sum_{j=1}^{m_{1}} X_{j} \phi_{j}
$$

if $X_{j} \phi_{j}$ exist for $j=1, \ldots, m_{1}$.

Now we use the notion of P-differentiability do introduce the G-regular surfaces.

Definition 2.2. $S \subset \mathbf{G}$ is a $k$-codimensional $\mathbf{G}$-regular surface if for every $p \in S$ there are a neighbourhood $\mathcal{U}$ of $p$ and a function $f=\left(f_{1}, \ldots, f_{k}\right) \in \mathbf{C}_{\mathbf{G}}^{1}\left(\mathcal{U}, \mathbf{R}^{k}\right)$ such that

$$
S \cap \mathcal{U}=\{q \in \mathcal{U}: f(q)=0\}
$$

and $d_{\mathbf{P}} f(q)$ is surjective, or equivalently if the $\left(k \times m_{1}\right)$ matrix $\nabla_{\mathbf{G}} f(q)$ has rank $k$, for all $q \in \mathcal{U}$.

The class of G-regular surfaces is different from the class of Euclidean regular surfaces. In [26], the authors give an example of $\mathbf{H}^{1}$-regular surfaces, in $\mathbf{H}^{1}$ identified with $\mathbf{R}^{3}$, that are (Euclidean) fractal sets. Conversely, there are continuously differentiable 2-submanifolds in $\mathbf{R}^{3}$ that are not $\mathbf{H}^{1}$-regular surfaces (see [18, Remark 6.2] and [3, Corollary 5.11]).

In the setting of Carnot groups, there is a natural definition of bounded variation functions and of finite perimeter sets (see [24] or [37] and the bibliography therein). We say that $f: \mathcal{U} \rightarrow \mathbf{R}$ is of bounded $\mathbf{G}$-variation in an open set $\mathcal{U} \subset \mathbf{G}$ and we write $f \in B V_{\mathbf{G}}(\mathcal{U})$, if $f \in \mathcal{L}^{1}(\mathcal{U})$ and

$\left\|\nabla_{\mathbf{G}} f\right\|(\mathcal{U}):=\sup \left\{\int_{\mathcal{U}} f \operatorname{div}_{\mathbf{G}} \phi d \mathcal{L}^{N}: \phi \in \mathbf{C}_{c}^{1}(\mathcal{U}, H \mathbf{G}),|\phi(p)| \leq 1\right.$ for all $\left.p \in \mathcal{U}\right\}<+\infty$.

The space $B V_{\mathbf{G}, \text { loc }}(\mathcal{U})$ is defined in the usual way.

In the setting of Carnot groups, the structure theorem for $B V_{\mathbf{G}}$ functions reads as follows.

Theorem 2.1. [8] If $f \in B V_{\mathbf{G}, l o c}(\mathcal{U})$ then $\left\|\nabla_{\mathbf{G}} f\right\|$ is a Radon measure on $\mathcal{U}$. Moreover, there is a $\left\|\nabla_{\mathbf{G}} f\right\|$ measurable horizontal section $\sigma_{f}: \mathcal{U} \rightarrow H \mathbf{G}$ such that $\left|\sigma_{f}(p)\right|=1$ for $\left\|\nabla_{\mathbf{G}} f\right\|$-a.e. $p \in \mathcal{U}$ and

$$
\int_{\mathcal{U}} f \operatorname{div}_{\mathbf{G}} \xi d \mathcal{L}^{N}=\int_{\mathcal{U}}\left\langle\xi, \sigma_{f}\right\rangle d\left\|\nabla_{\mathbf{G}} f\right\|
$$

for every $\xi \in \mathbf{C}_{c}^{1}(\mathcal{U}, H \mathbf{G})$. Finally the notion of gradient $\nabla_{\mathbf{G}}$ can be extended from regular functions to functions $f \in B V_{\mathbf{G}}$ defining $\nabla_{\mathbf{G}} f$ as the vector valued measure

$$
\nabla_{\mathbf{G}} f:=-\sigma_{f}\left\llcorner\left\|\nabla_{\mathbf{G}} f\right\|=\left(-\left(\sigma_{f}\right)_{1} \mathbf{L}\left\|\nabla_{\mathbf{G}} f\right\|, \ldots,-\left(\sigma_{f}\right)_{m_{1}}\left\llcorner\left\|\nabla_{\mathbf{G}} f\right\|\right),\right.\right.
$$

where $\left(\sigma_{f}\right)_{i}$ are the components of $\sigma_{f}$ with respect to the base $X_{i}$.

A set $\mathcal{E} \subset \mathbf{G}$ has locally finite $\mathbf{G}$-perimeter, or is a $\mathbf{G}$-Caccioppoli set, if $\chi_{\mathcal{E}} \in$ $B V_{\mathbf{G}, l o c}(\mathbf{G})$, where $\chi_{\mathcal{E}}$ is the characteristic function of the set $\mathcal{E}$. In this case the 
measure $\left\|\nabla_{\mathbf{G}} \chi_{\mathcal{E}}\right\|$ is called the $\mathbf{G}$-perimeter measure of $\mathcal{E}$ and is denoted by $|\partial \mathcal{E}|_{\mathbf{G}}$. Moreover we call generalized inward normal of $\partial \mathcal{E}$ in $\Omega$ the vector

$$
\nu_{\mathcal{E}}(p):=-\sigma_{\chi \mathcal{E}}(p) .
$$

Fundamental estimates in geometric measure theory are the so-called relative and global isoperimetric inequalities for Caccioppoli sets. The proof is established in [24], Theorem 1.18.

Theorem 2.2. There exists a constant $C>0$ such that for any G-Caccippoli set $E \subset \mathbf{G}$, for every $p \in \mathbf{G}$ and $r>0$

$$
\min \left\{\mathcal{L}^{N}(E \cap \mathcal{U}(p, r)), \mathcal{L}^{N}(\mathcal{U}(p, r)-E)\right\}^{(\mathfrak{q}-1) / \mathfrak{q}} \leq C|\partial E|_{\mathbf{G}}(\mathcal{U}(p, r))
$$

and

$$
\min \left\{\mathcal{L}^{N}(E), \mathcal{L}^{N}(\mathbf{G}-E)\right\}^{(\mathfrak{q}-1) / \mathfrak{q}} \leq C|\partial E|_{\mathbf{G}}(\mathbf{G}),
$$

where $\mathfrak{q}$ is homogeneous dimension of $\mathbf{G}$ defined in Section 2.1.

The perimeter measure is concentrated in a subset of topological boundary of $E$, the so-called reduced boundary $\partial_{\mathbf{G}}^{*} E$.

Definition 2.3. (Reduced boundary) Let $E \subset \mathbf{G}$ be a $\mathbf{G}$-Caccioppoli set. We say that $p \in \partial_{\mathbf{G}}^{*} E$ if

(1) $|\partial E|_{\mathbf{G}}(\mathcal{U}(p, r))>0$, for all $r>0$,

(2) there exists $\lim _{r \rightarrow 0} f_{\mathcal{U}(p, r)} \nu_{E} d|\partial E|_{\mathbf{G}}$,

(3) $\left.\left|\lim _{r \rightarrow 0} f_{\mathcal{U}(p, r)} \nu_{E} d\right| \partial E\right|_{\mathbf{G}} \mid=1$.

The reduced boundary of a set $E \subset \mathbf{G}$ is invariant under group translations, i.e.

$$
q \in \partial_{\mathbf{G}}^{*} E \quad \text { if and only if } \tau_{p} q \in \partial_{\mathbf{G}}^{*}\left(\tau_{p} E\right)
$$

and also $\nu_{E}(q)=\nu_{\tau_{p} E}\left(\tau_{p} q\right)$.

Lemma 2.3. [1, Differentiation Lemma] If $E \subset \mathbf{G}$ is a $\mathbf{G}$-Caccioppoli set, then

$$
\lim _{r \rightarrow 0} f_{\mathcal{U}(p, r)} \nu_{E} d|\partial E|_{\mathbf{G}}=\nu_{E}(p), \quad \text { for }|\partial E|_{\mathbf{G}} \text {-a.e. } p,
$$

hence $|\partial E|_{\mathbf{G}}$ is concentrated on the reduced boundary $\partial_{\mathbf{G}}^{*} E$.

The following results are only proven for specific choice of metric: the metric $d_{\infty}$. Theorem 2.4 states that the perimeter measure equals a constant times the spherical $(\mathfrak{q}-1)$-dimensional Hausdorff measure restricted to the reduced boundary.

Theorem 2.4. [23, Theorem 4.18] Let $\mathbf{G}$ be a Carnot group of step 2, endowed with a distance $d_{\infty}$. If $E \subset \mathbf{G}$ is a $\mathbf{G}$-Caccioppoli set, then

$$
|\partial E|_{\mathbf{G}}=c \mathcal{S}^{\mathfrak{q}-1}\left\llcorner\partial_{\mathbf{G}}^{*} E,\right.
$$

where $\mathfrak{q}$ is homogeneous dimension of $\mathbf{G}$ defined in Section 2.1.

At each point of the reduced boundary of a G-Caccioppoli set there is a (generalized) tangent group:

Theorem 2.5. [20, Blow-up Theorem] Let $\mathbf{G}$ be a Carnot group of step 2, endowed with a distance $d_{\infty}$, and let $E \subset \mathbf{G}$ be a set with locally finite $\mathbf{G}$-perimeter. If $p \in \partial_{\mathbf{G}}^{*} E$, then

$$
\lim _{r \rightarrow 0} \chi_{E_{r, p}}=\chi_{S_{\mathbf{G}}^{+}\left(\nu_{E}(p)\right)} \quad \text { in } \mathcal{L}_{\text {loc }}^{1}(\mathbf{G})
$$


where $E_{r, p}:=\delta_{1 / r}\left(\tau_{p^{-1}} E\right)=\left\{q: \tau_{p}\left(\delta_{r}(q)\right) \in E\right\}$ and

$$
S_{\mathbf{G}}^{+}\left(\nu_{E}(p)\right):=\left\{q=\left(q^{1}, q^{2}\right) \in \mathbf{G} \mid\left\langle\nu_{E}(p), q^{1}\right\rangle \geq 0\right\} .
$$

Moreover, for all $\delta>0$

$$
\lim _{r \rightarrow 0}\left|\partial E_{r, p}\right|_{\mathbf{G}}(\mathcal{U}(0, \delta))=\left|\partial S_{\mathbf{G}}^{+}\left(\nu_{E}(p)\right)\right|_{\mathbf{G}}(\mathcal{U}(0, \delta))
$$

and

$$
\left|\partial S_{\mathbf{G}}^{+}\left(\nu_{E}(p)\right)\right|_{\mathbf{G}}(\mathcal{U}(0, \delta))=\mathcal{H}^{N-1}\left(T_{\mathbf{G}}^{g}\left(\nu_{E}(0)\right) \cap \mathcal{U}(0, \delta)\right)
$$

where $\mathcal{H}^{N-1}$ denotes the Euclidean Hausdorff measure and $T_{\mathbf{G}}^{g}\left(\nu_{E}(0)\right):=\{q=$ $\left.\left(q^{1}, q^{2}\right) \in \mathbf{G} \mid\left\langle\nu_{E}(0), Q^{1}\right\rangle=0\right\}$ is the topological boundary of $S_{\mathbf{G}}^{+}\left(\nu_{E}(0)\right)$.

Finally, as it is usual in the literature, we can also define the measure theoretic boundary $\partial_{*, \mathbf{G}} E$ :

Definition 2.4. Let $E \subset \mathbf{G}$ be a measurable set. We say that $p$ belongs to measure theoretic boundary $\partial_{*, \mathbf{G}} E$ of $E$ if

$$
\limsup _{r \rightarrow 0^{+}} \frac{\mathcal{L}^{N}(E \cap \mathcal{U}(p, r))}{\mathcal{L}^{N}(\mathcal{U}(p, r))}>0 \quad \text { and } \quad \limsup _{r \rightarrow 0^{+}} \frac{\mathcal{L}^{N}\left(E^{c} \cap \mathcal{U}(p, r)\right)}{\mathcal{L}^{N}(\mathcal{U}(p, r))}>0 .
$$

If $E \subset \mathbf{G}$ is $\mathbf{G}$-Caccioppoli set, then

$$
\partial_{\mathbf{G}}^{*} E \subset \partial_{*, \mathbf{G}} E \subset \partial E .
$$

Moreover, $\mathcal{S}^{\mathfrak{q}-1}\left(\partial_{*, \mathbf{G}} E-\partial_{\mathbf{G}}^{*} E\right)=0$.

\subsection{Complementary subgroups and graphs.}

Definition 2.5. We say that $\mathbf{W}$ and $\mathbf{M}$ are complementary subgroups in $\mathbf{G}$ if $\mathbf{W}$ and $\mathbf{M}$ are homogeneous subgroups ${ }^{1}$ of $\mathbf{G}$ such that $\mathbf{W} \cap \mathbf{M}=\{0\}$ and

$$
\mathrm{G}=\mathrm{W} \cdot \mathbf{M} \text {. }
$$

By this we mean that for every $p \in \mathbf{G}$ there are $p_{\mathbf{W}} \in \mathbf{W}$ and $p_{\mathbf{M}} \in \mathbf{M}$ such that $p=p_{\mathbf{W}} p_{\mathbf{M}}$.

The elements $p_{\mathbf{W}} \in \mathbf{W}$ and $p_{\mathbf{M}} \in \mathbf{M}$ such that $p=p_{\mathbf{W}} \cdot p_{\mathbf{M}}$ are unique because of $\mathbf{W} \cap \mathbf{M}=\{0\}$ and are denoted components of $p$ along $\mathbf{W}$ and $\mathbf{M}$ or projections of $p$ on $\mathbf{W}$ and $\mathbf{M}$. The projection maps $\mathbf{P}_{\mathbf{W}}: \mathbf{G} \rightarrow \mathbf{W}$ and $\mathbf{P}_{\mathbf{M}}: \mathbf{G} \rightarrow \mathbf{M}$ defined

$$
\mathbf{P}_{\mathbf{W}}(p)=p_{\mathbf{W}}, \quad \mathbf{P}_{\mathbf{M}}(p)=p_{\mathbf{M}}, \quad \text { for all } p \in \mathbf{G}
$$

are polynomial functions (see Proposition 2.2.14 in [17]) if we identify $\mathbf{G}$ with $\mathbf{R}^{N}$, hence are $\mathbf{C}^{\infty}$. Nevertheless in general they are not Lipschitz maps, when $\mathbf{W}$ and $\mathbf{M}$ are endowed with the restriction of the left invariant distance $d$ of $\mathbf{G}$ (see Example 2.2 .15 in [17]).

Remark 2.6. The stratification of $\mathbf{G}$ induces a stratifications on the complementary subgroups $\mathbf{W}$ and $\mathbf{M}$. If $\mathbf{G}=\mathbf{G}^{1} \oplus \cdots \oplus \mathbf{G}^{\kappa}$ then also $\mathbf{W}=\mathbf{W}^{1} \oplus \cdots \oplus \mathbf{W}^{\kappa}$, $\mathbf{M}=\mathbf{M}^{1} \oplus \cdots \oplus \mathbf{M}^{\kappa}$ and $\mathbf{G}^{i}=\mathbf{W}^{i} \oplus \mathbf{M}^{i}$. A subgroup is horizontal if it is contained in the first layer $\mathbf{G}^{1}$. If $\mathbf{M}$ is horizontal then the complementary subgroup $\mathbf{W}$ is normal.

\footnotetext{
${ }^{1}$ An homogeneous subgroup $\mathbf{W}$ of $\mathbf{G}$ is a Lie subgroup such that $\delta_{\lambda} a \in \mathbf{W}$ for every $a \in \mathbf{W}$ and $\lambda>0$.
} 
Proposition 2.7. [4, Proposition 3.2] If $\mathbf{W}$ and $\mathbf{M}$ are complementary subgroups in $\mathbf{G}$ there is $c_{0}=c_{0}(\mathbf{W}, \mathbf{M}) \in(0,1)$ such that for each $p_{\mathbf{W}} \in \mathbf{W}$ and $p_{\mathbf{M}} \in \mathbf{M}$

$$
c_{0}\left(\left\|p_{\mathbf{W}}\right\|+\left\|p_{\mathbf{M}}\right\|\right) \leq\left\|p_{\mathbf{W}} p_{\mathbf{M}}\right\| \leq\left\|p_{\mathbf{W}}\right\|+\left\|p_{\mathbf{M}}\right\|
$$

Definition 2.6. We say that $S \subset \mathbf{G}$ is a left intrinsic graph or more simply an intrinsic graph if there are complementary subgroups $\mathbf{W}$ and $\mathbf{M}$ in $\mathbf{G}$ and $\phi: \mathcal{O} \subset$ $\mathbf{W} \rightarrow \mathbf{M}$ such that

$$
S=\operatorname{graph}(\phi):=\{a \phi(a): a \in \mathcal{O}\} .
$$

Observe that, by uniqueness of the components along $\mathbf{W}$ and $\mathbf{M}$, if $S=\operatorname{graph}(\phi)$ then $\phi$ is uniquely determined among all functions from $\mathbf{W}$ to $\mathbf{M}$.

We call graph map of $\phi$, the function $\Phi: \mathcal{O} \rightarrow \mathbf{G}$ defined as

$$
\Phi(a):=a \cdot \phi(a) \text { for all } a \in \mathcal{O} \text {. }
$$

Hence $S=\Phi(\mathcal{O})$ is equivalent to $S=\operatorname{graph}(\phi)$.

The concept of intrinsic graph is preserved by translation and dilation, i.e.

Proposition 2.8. [17, Proposition 2.2.18] If $S$ is an intrinsic graph then, for all $\lambda>0$ and for all $q \in \mathbf{G}, q \cdot S$ and $\delta_{\lambda} S$ are intrinsic graphs. In particular, if $S=\operatorname{graph}(\phi)$ with $\phi: \mathcal{O} \subset \mathbf{W} \rightarrow \mathbf{M}$, then

(1) For all $\lambda>0$,

$$
\delta_{\lambda}(\operatorname{graph}(\phi))=\operatorname{graph}\left(\phi_{\lambda}\right)
$$

where $\phi_{\lambda}: \delta_{\lambda} \mathcal{O} \subset \mathbf{W} \rightarrow \mathbf{M}$ and $\phi_{\lambda}(a):=\delta_{\lambda} \phi\left(\delta_{1 / \lambda} a\right)$, for $a \in \delta_{\lambda} \mathcal{O}$.

(2) For any $q \in \mathbf{G}$,

$$
q \cdot \operatorname{graph}(\phi)=\operatorname{graph}\left(\phi_{q}\right)
$$

where $\phi_{q}: \mathcal{O}_{q} \subset \mathbf{W} \rightarrow \mathbf{M}$ is defined as $\phi_{q}(a):=\left(\mathbf{P}_{\mathbf{M}}\left(q^{-1} a\right)\right)^{-1} \phi\left(\mathbf{P}_{\mathbf{W}}\left(q^{-1} a\right)\right)$, for all $a \in \mathcal{O}_{q}:=\left\{a: \mathbf{P}_{\mathbf{W}}\left(q^{-1} a\right) \in \mathcal{O}\right\}$.

\subsection{Intrinsic differentiability.}

Definition 2.7. Let $\mathbf{W}$ and $\mathbf{M}$ be complementary subgroups in $\mathbf{G}$. Then $\ell: \mathbf{W} \rightarrow \mathbf{M}$ is intrinsic linear if $\ell$ is defined on all of $\mathbf{W}$ and if graph $(\ell)$ is a homogeneous subgroup of $\mathbf{G}$.

We use intrinsic linear functions to define intrinsic differentiability as in the usual definition of differentiability.

Definition 2.8. Let $\mathbf{W}$ and $\mathbf{M}$ be complementary subgroups in $\mathbf{G}$ and let $\phi: \mathcal{O} \subset \mathbf{W} \rightarrow \mathbf{M}$ with $\mathcal{O}$ open in $\mathbf{W}$. For $a \in \mathcal{O}$, let $p:=a \cdot \phi(a)$ and $\phi_{p^{-1}}: \mathcal{O}_{p^{-1}} \subset$ $\mathbf{W} \rightarrow \mathbf{M}$ be the shifted function defined in Proposition 2.8.

(1) We say that $\phi$ is intrinsic differentiable in $a$ if the shifted function $\phi_{p^{-1}}$ is intrinsic differentiable in 0, i.e. if there is an intrinsic linear $d \phi_{a}: \mathbf{W} \rightarrow \mathbf{M}$ such that

$$
\lim _{r \rightarrow 0^{+}} \sup _{0<\|b\|<r} \frac{\left\|d \phi_{a}(b)^{-1} \phi_{p^{-1}}(b)\right\|}{\|b\|}=0 .
$$

The function $d \phi_{a}$ is the intrinsic differential of $\phi$ at $a$.

(2) We say that $\phi$ is uniformly intrinsic differentiable in $a_{0} \in \mathcal{O}$ or $\phi$ is u.i.d. in $a_{0}$ if there exist an intrinsic linear function $d \phi_{a_{0}}: \mathbf{W} \rightarrow \mathbf{M}$ such that

$$
\lim _{r \rightarrow 0^{+}} \sup _{\left\|a_{0}^{-1} a\right\|<r} \sup _{0<\|b\|<r} \frac{\left\|d \phi_{a_{0}}(b)^{-1} \phi_{p^{-1}}(b)\right\|}{\|b\|}=0 .
$$

Analogously, $\phi$ is u.i.d. in $\mathcal{O}$ if it is u.i.d. in every point of $\mathcal{O}$. 
Remark 2.9. Definition 2.8 is a natural one because of the following observations.

(i) If $\phi$ is intrinsic differentiable in $a \in \mathcal{O}$, there is a unique intrinsic linear function $d \phi_{a}$ satisfying (1). Moreover $\phi$ is continuous at $a$. (See Theorem 3.2.8 and Proposition 3.2.3 in [16]).

(ii) The notion of intrinsic differentiability is invariant under group translations. Precisely, let $p:=a \phi(a), q:=b \phi(b)$, then $\phi$ is intrinsic differentiable in $a$ if and only if $\phi_{q p^{-1}}:=\left(\phi_{p^{-1}}\right)_{q}$ is intrinsic differentiable in $b$.

(iii) We recall that in Definition 3.16 in [4] the authors give another notion of uniformly intrinsic differentiable map but these notions are equivalent. Indeed, Proposition 3.7 (3) in [11] implies that u.i.d. mappings in the sense of Definition 2.8 satisfy the second point of Definition 3.16 in [4]. Moreover, it is clear, that if $\phi$ is u.i.d. then it is intrinsic differentiable (i.e. the first point of Definition 3.16 in [4]). Finally the first and third point of Definition 3.16 in [4] tells us that i.d. mappings in the sense of Definition 3.16 in [4] satisfy Definition 2.8 (2).

The analytic definition of intrinsic differentiability of Definition 2.8 has an equivalent geometric formulation. Indeed intrinsic differentiability in one point is equivalent to the existence of a tangent subgroup to the graph. We recall the definition of tangent subgroup:

Definition 2.9. [16, Definition 3.2.6] Let $\mathbf{W}, \mathbf{M}$ be complementary subgroups in $\mathbf{G}, \phi: \mathcal{O} \rightarrow \mathbf{M}$ with $\mathcal{O}$ relatively open in $\mathbf{W}$ and $\mathbf{T}$ be a homogeneous subgroup of $\mathbf{G}$. Let $a \in \mathcal{O}$ and $p=a \cdot \phi(a)$. We say that $p \cdot \mathbf{T}$ is the tangent coset to graph $(\phi)$ in $p$ if for all $\epsilon>0$ there is $\lambda(\epsilon)>0$ such that

$$
\operatorname{graph}(\phi) \cap\left\{q \in \mathbf{G}:\left\|\mathbf{P}_{\mathbf{W}}\left(p^{-1} q\right)\right\|<\lambda(\epsilon)\right\} \subset p \cdot\{q \in \mathbf{G}: \operatorname{dist}(q, \mathbf{T}) \leq \epsilon\|q\|\},
$$

Theorem 2.10. [16, Theorem 3.2.8] Let W, M be complementary subgroups in $\mathbf{G}$ and let $\phi: \mathcal{O} \rightarrow \mathbf{M}$ with $\mathcal{O}$ relatively open in $\mathbf{W}$. If $\phi$ is intrinsic differentiable in $a \in \mathcal{O}$, set $\mathbf{T}:=\operatorname{graph}\left(d \phi_{a}\right)$. Then

(1) $\mathbf{T}$ is a homogeneous subgroup of $\mathbf{G}$;

(2) $\mathbf{T}$ and $\mathbf{M}$ are complementary subgroups in $\mathbf{G}$;

(3) $p \cdot \mathbf{T}$ is the tangent coset to graph $(\phi)$ in $p:=a \phi(a)$.

Conversely, if $p:=a \phi(a) \in \operatorname{graph}(\phi)$ and if there is $\mathbf{T}$ such that (1), (2), (3) hold, then $\phi$ is intrinsic differentiable in $a$ and the differential $d \phi_{a}: \mathbf{W} \rightarrow \mathbf{M}$ is the unique intrinsic linear function such that $\mathbf{T}:=\operatorname{graph}\left(d \phi_{a}\right)$.

From now on we study the notions of intrinsic differentiability and of uniform intrinsic differentiability for functions $\phi: \mathbf{W} \rightarrow \mathbf{H}$ when $\mathbf{H}$ is a horizontal subgroup. When $\mathbf{H}$ is horizontal, $\mathbf{W}$ is always a normal subgroup since, as observed in Remark 2.6, it contains the whole strata $\mathbf{G}^{2}, \ldots, \mathbf{G}^{\kappa}$. In this case, the more explicit form of the shifted function $\phi_{p^{-1}}$ allows a more explicit form of the equations in Definition 2.8 .

Proposition 2.11. [11, Theorem 3.5] Let $\mathbf{H}$ and $\mathbf{W}$ be complementary subgroups of $\mathbf{G}, \mathcal{O}$ open in $\mathbf{W}$ and $\mathbf{H}$ horizontal. Then $\phi: \mathcal{O} \subset \mathbf{W} \rightarrow \mathbf{H}$ is intrinsic differentiable in $a_{0} \in \mathcal{O}$ if and only if there is an intrinsic linear $d \phi_{a_{0}}: \mathbf{W} \rightarrow \mathbf{H}$ such that

$$
\lim _{r \rightarrow 0^{+}} \sup _{0<\left\|a_{0}^{-1} b\right\|<r} \frac{\left\|\phi(b)-\phi\left(a_{0}\right)-d \phi_{a_{0}}\left(a_{0}^{-1} b\right)\right\|}{\left\|\phi\left(a_{0}\right)^{-1} a_{0}^{-1} b \phi\left(a_{0}\right)\right\|}=0 .
$$


Analogously, $\phi$ is uniformly intrinsic differentiable in $a_{0} \in \mathcal{O}$, or $\phi$ is u.i.d. in $a_{0} \in \mathcal{O}$, if there is an intrinsic linear $d \phi_{a_{0}}: \mathbf{W} \rightarrow \mathbf{H}$ such that

$$
\lim _{r \rightarrow 0^{+}} \sup _{a, b} \frac{\left\|\phi(b)-\phi(a)-d \phi_{a_{0}}\left(a^{-1} b\right)\right\|}{\left\|\phi(a)^{-1} a^{-1} b \phi(a)\right\|}=0
$$

where $r$ is small enough so that $\mathcal{U}\left(a_{0}, 2 r\right) \subset \mathcal{O}$ and the supremum is for $\left\|a_{0}^{-1} a\right\|<$ $r, 0<\left\|a^{-1} b\right\|<r$. Finally, if $k<m_{1}$ is the dimension of $\mathbf{H}$, and if, without loss of generality, we assume that

$$
\mathbf{H}=\left\{p: p_{k+1}=\cdots=p_{N}=0\right\} \quad \mathbf{W}=\left\{p: p_{1}=\cdots=p_{k}=0\right\}
$$

then there is a $k \times\left(m_{1}-k\right)$ matrix, here denoted as $\nabla^{\phi} \phi\left(a_{0}\right)$, such that

$$
d \phi_{a_{0}}(b)=\left(\nabla^{\phi} \phi\left(a_{0}\right)\left(b_{k+1}, \ldots, b_{m_{1}}\right)^{T}, 0, \ldots, 0\right),
$$

for all $b=\left(b_{1}, \ldots, b_{N}\right) \in \mathbf{W}$. The matrix $\nabla^{\phi} \phi\left(a_{0}\right)$ is called the intrinsic horizontal Jacobian of $\phi$ in $a_{0}$ or the intrinsic horizontal gradient or even the intrinsic gradient if $k=1$.

Observe that u.i.d. functions do exist. In particular, when $\mathbf{H}$ is a horizontal subgroup, $\mathbf{H}$ valued euclidean $\mathbf{C}^{1}$ functions are u.i.d.

Theorem 2.12. [11, Theorem 4.7] If $\mathbf{W}$ and $\mathbf{H}$ are complementary subgroups of a step $\kappa$ Carnot group $\mathbf{G}$ with $\mathbf{H}$ horizontal and $k$ dimensional. If $\mathcal{O}$ is open in $\mathbf{W}$ and $\phi: \mathcal{O} \subset \mathbf{W} \rightarrow \mathbf{H}$ is such that $\phi \in \mathbf{C}^{1}(\mathcal{O}, \mathbf{H})$ then $\phi$ is u.i.d. in $\mathcal{O}$.

There is a comparison between G-regular surfaces (see Definition 2.2) and the uniformly intrinsic differentiable maps, proved in [3, Theorem 1.2] for the Heisenberg groups and in [11, Theorem 4.1] for Carnot groups of step $\kappa$.

Theorem 2.13. Let $\mathbf{W}$ and $\mathbf{H}$ be complementary subgroups of a step $\kappa$ Carnot group $\mathbf{G}$ with $\mathbf{H}$ horizontal and $k$ dimensional. Let $\mathcal{O}$ be open in $\mathbf{W}, \phi: \mathcal{O} \subset \mathbf{W} \rightarrow \mathbf{H}$ and $S:=\operatorname{graph}(\phi)$. Then for every $a_{0} \in \mathcal{O}$ the following are equivalent:

(1) there are a neighbourhood $\mathcal{U}$ of $a_{0} \cdot \phi\left(a_{0}\right)$ and $f \in \mathbf{C}_{\mathbf{G}}^{1}\left(\mathcal{U} ; \mathbf{R}^{k}\right)$ such that

$$
\begin{aligned}
& S \cap \mathcal{U}=\{p \in \mathcal{U}: f(p)=0\}, \\
& d_{\mathbf{P}} f(Q)_{\mid \mathbf{H}}: \mathbf{H} \rightarrow \mathbf{R}^{k} \text { is bijective for all } Q \in \mathcal{U},
\end{aligned}
$$

and $Q \mapsto\left(d_{\mathbf{P}} f(q)_{\mid \mathbf{H}}\right)^{-1}$ is continuous.

(2) $\phi$ is u.i.d. in a neighbourhood $\mathcal{O}^{\prime} \subset \mathcal{O}$ of $a_{0}$.

Moreover, if (1) or equivalently (2), hold then, for all $a \in \mathcal{O}$ the intrinsic differential $d \phi_{a}$ is

$$
d \phi_{a}=-\left(d_{\mathbf{P}} f(a \phi(a))_{\mid \mathbf{H}}\right)^{-1} \circ d_{\mathbf{P}} f(a \phi(a))_{\mid \mathbf{W}} .
$$

Remark 2.14. If, without loss of generality, we choose a base $X_{1}, \ldots, X_{N}$ of $\mathfrak{g}$ such that $X_{1}, \ldots, X_{k}$ are horizontal vector fields, $\mathbf{H}=\exp \left(\operatorname{span}\left\{X_{1}, \ldots, X_{k}\right\}\right)$ and $\mathbf{W}=\exp \left(\operatorname{span}\left\{X_{k+1}, \ldots, X_{N}\right\}\right)$, then

$$
\mathbf{H}=\left\{p: p_{k+1}=\cdots=p_{N}=0\right\}, \quad \mathbf{W}=\left\{p: p_{1}=\cdots=p_{k}=0\right\},
$$

and, if $f=\left(f_{1}, \ldots, f_{k}\right)$, then $\nabla_{\mathbf{G}} f=\left(\mathcal{M}_{1} \mid \mathcal{M}_{2}\right)$, where

$$
\mathcal{M}_{1}:=\left(\begin{array}{cccc}
X_{1} f_{1} & \ldots & X_{k} f_{1} \\
\vdots & \ddots & \vdots \\
X_{1} f_{k} & \ldots & X_{k} f_{k}
\end{array}\right), \quad \mathcal{M}_{2}:=\left(\begin{array}{cccc}
X_{k+1} f_{1} & \ldots & X_{m_{1}} f_{1} \\
\vdots & \ddots & \vdots \\
X_{k+1} f_{k} & \ldots & X_{m_{1}} f_{k}
\end{array}\right) .
$$


Moreover, for all $a \in \mathcal{O}$, for all $q$ in a neighborhood $\mathcal{U}$ of $a \phi(a)$ and for all $p \in \mathbf{G}$

$$
\left(d_{\mathbf{P}} f(q)\right)(p)=\left(\nabla_{\mathbf{G}} f(q)\right) p^{1}
$$

and the intrinsic differential is

$$
\begin{aligned}
d \phi_{a}(b) & =\left(\left(\nabla^{\phi} \phi(a)\right)\left(b_{k+1}, \ldots, b_{m_{1}}\right)^{T}, 0, \ldots, 0\right) \\
& =\left(\left(-\mathcal{M}_{1}(a \phi(a))^{-1} \mathcal{M}_{2}(a \phi(a))\right)\left(b_{k+1}, \ldots, b_{m_{1}}\right)^{T}, 0, \ldots, 0\right),
\end{aligned}
$$

for all $b=\left(b_{1}, \ldots, b_{N}\right) \in \mathbf{W}$.

2.5. Intrinsic Lipschitz function. The following notion of intrinsic Lipschitz function appeared for the first time in [21] and was studied in [5, 6, 16, 17, 22, 36]. Intrinsic Lipschitz functions play the same role as Lipschitz functions in Euclidean context.

Definition 2.10. Let $\mathbf{W}, \mathbf{H}$ be complementary subgroups in $\mathbf{G}, q \in \mathbf{G}$ and $\beta \geq 0$. We can define the cones $C_{\mathbf{W}, \mathbf{H}}(q, \beta)$ with base $\mathbf{W}$ and axis $\mathbf{H}$, vertex $q$, opening $\beta$ as

$$
C_{\mathbf{W}, \mathbf{H}}(q, \beta)=q \cdot C_{\mathbf{W}, \mathbf{H}}(0, \beta),
$$

where $C_{\mathbf{W}, \mathbf{H}}(0, \beta)=\left\{p:\left\|p_{\mathbf{W}}\right\| \leq \beta\left\|p_{\mathbf{H}}\right\|\right\}$. then

For all $\lambda>0$ we have that $\delta_{\lambda}\left(C_{\mathbf{W}, \mathbf{H}}\left(0, \beta_{1}\right)\right)=C_{\mathbf{W}, \mathbf{H}}\left(0, \beta_{1}\right)$ and if $0<\beta_{1}<\beta_{2}$,

$$
C_{\mathbf{W}, \mathbf{H}}\left(q, \beta_{1}\right) \subset C_{\mathbf{W}, \mathbf{H}}\left(q, \beta_{2}\right) .
$$

Now we introduce the basic definitions of this paragraph.

Definition 2.11. Let $\mathbf{W}, \mathbf{H}$ are complementary subgroups in $\mathbf{G}$. We say that $\phi: \mathcal{O} \subset \mathbf{W} \rightarrow \mathbf{H}$ is intrinsic $C_{L^{-}}$Lipschitz in $\mathcal{O}$ for some $C_{L} \geq 0$ if for all $C_{1}>C_{L}$

$$
C_{\mathbf{W}, \mathbf{H}}\left(p, 1 / C_{1}\right) \cap \operatorname{graph}(\phi)=\{p\} \quad \text { for all } p \in \operatorname{graph}(\phi) .
$$

The Lipschitz constant of $\phi$ in $\mathcal{O}$ is the infimum of the $C_{1}>0$ such that (8) holds. An intrinsic Lipschitz (continuous) function, with Lipschitz constant $C_{L}>0$, is called a $C_{L}$-Lipschitz function. We will call a set $S \subset \mathbf{G}$ an intrinsic Lipschitz graph if there exists an intrinsic Lipschitz function $\phi: \mathcal{O} \subset \mathbf{W} \rightarrow \mathbf{H}$ such that $S=\operatorname{graph}(\phi)$ for suitable complementary subgroups $\mathbf{W}$ and $\mathbf{H}$.

We observe that the geometric definition of intrinsic Lipschitz graphs has equivalent analytic forms (see Proposition 3.1.3. in [17]):

Proposition 2.15. Let $\mathbf{W}, \mathbf{H}$ be complementary subgroups in $\mathbf{G}, \phi: \mathcal{O} \subset \mathbf{W} \rightarrow$ $\mathbf{H}$ and $C_{L}>0$. Then the following statements are equivalent:

(1) $\phi$ is intrinsic $C_{L}$-Lipschitz in $\mathcal{O}$.

(2) $\left\|\mathbf{P}_{\mathbf{H}}\left(q^{-1} q^{\prime}\right)\right\| \leq C_{L}\left\|\mathbf{P}_{\mathbf{W}}\left(q^{-1} q^{\prime}\right)\right\|$ for all $q, q^{\prime} \in \operatorname{graph}(\phi)$.

(3) $\left\|\phi_{q^{-1}}(a)\right\| \leq C_{L}\|a\|$ for all $q \in \operatorname{graph}(\phi)$ and $a \in \mathcal{O}_{q^{-1}}$.

If $\phi: \mathcal{O} \subset \mathbf{W} \rightarrow \mathbf{H}$ is intrinsic $C_{L}$-Lipschitz in $\mathcal{O}$ then it is continuous. Indeed if $\phi(0)=0$ then by the condition 3. of Proposition $2.15 \phi$ is continuous in 0 . To prove the continuity in $a \in \mathcal{O}$, observe that $\phi_{q^{-1}}$ is continuous in 0 , where $q=a \phi(a)$.

Remark 2.16. In this paper we are interested mainly in the special case when $\mathbf{H}$ is a horizontal subgroup and consequently $\mathbf{W}$ is a normal subgroup. Under these assumptions, for all $p=a \phi(a), q=b \phi(b) \in \operatorname{graph}(\phi)$ we have

$$
\mathbf{P}_{\mathbf{H}}\left(p^{-1} q\right)=\phi(a)^{-1} \phi(b), \quad \mathbf{P}_{\mathbf{W}}\left(p^{-1} q\right)=\phi(a)^{-1} a^{-1} b \phi(a) .
$$


Hence, if $\mathbf{H}$ is a horizontal subgroup, $\phi: \mathcal{O} \subset \mathbf{W} \rightarrow \mathbf{H}$ is intrinsic Lipschitz if there is $C_{L}>0$ such that

$$
\left\|\phi(a)^{-1} \phi(b)\right\| \leq C_{L}\left\|\phi(a)^{-1} a^{-1} b \phi(a)\right\| \quad \text { for all } a, b \in \mathcal{O} .
$$

Moreover, if $\phi$ is intrinsic Lipschitz then $\left\|\phi(a)^{-1} a^{-1} b \phi(a)\right\|$ is comparable with $\left\|p^{-1} q\right\|$. Indeed from (4)

$$
\begin{aligned}
c_{0}\left\|\phi(a)^{-1} a^{-1} b \phi(a)\right\| & \leq\left\|p^{-1} q\right\| \\
& \leq\left\|\phi(a)^{-1} a^{-1} b \phi(a)\right\|+\left\|\phi(a)^{-1} \phi(b)\right\| \\
& \leq\left(1+C_{L}\right)\left\|\phi(a)^{-1} a^{-1} b \phi(a)\right\| .
\end{aligned}
$$

The quantity $\left\|\phi(a)^{-1} a^{-1} b \phi(a)\right\|$, or better a symmetrized version of it, can play the role of a $\phi$ dependent, quasi distance on $\mathcal{O}$. See e.g. [3].

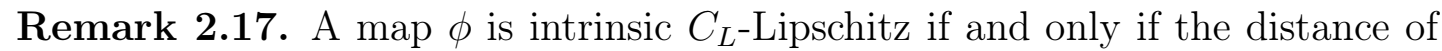
two points $q, q^{\prime} \in \operatorname{graph}(\phi)$ is bounded by the norm of the projection of $q^{-1} q^{\prime}$ on the domain $\mathcal{O}$. Precisely $\phi: \mathcal{O} \subset \mathbf{W} \rightarrow \mathbf{H}$ is intrinsic $C_{L}$-Lipschitz in $\mathcal{O}$ if and only if there exists a constant $C_{1}>0$ satisfying

$$
\left\|q^{-1} q^{\prime}\right\| \leq C_{1}\left\|\mathbf{P}_{\mathbf{W}}\left(q^{-1} q^{\prime}\right)\right\|
$$

for all $q, q^{\prime} \in \operatorname{graph}(\phi)$. Moreover the relations between $C_{1}$ and the Lipschitz constant $C_{L}$ of $\phi$ follow from (4). In fact if $\phi$ is intrinsic $C_{L^{-}}$-Lipschitz in $\mathcal{O}$ then

$$
\left\|q^{-1} q^{\prime}\right\| \leq\left\|\mathbf{P}_{\mathbf{W}}\left(q^{-1} q^{\prime}\right)\right\|+\left\|\mathbf{P}_{\mathbf{H}}\left(q^{-1} q^{\prime}\right)\right\| \leq\left(1+C_{L}\right)\left\|\mathbf{P}_{\mathbf{W}}\left(q^{-1} q^{\prime}\right)\right\|
$$

for all $q, q^{\prime} \in \operatorname{graph}(\phi)$. Conversely if $\left\|q^{-1} q^{\prime}\right\| \leq c_{0}\left(1+C_{L}\right)\left\|\mathbf{P}_{\mathbf{W}}\left(q^{-1} q^{\prime}\right)\right\|$ then

$$
\left\|\mathbf{P}_{\mathbf{H}}\left(q^{-1} q^{\prime}\right)\right\| \leq C_{L}\left\|\mathbf{P}_{\mathbf{W}}\left(q^{-1} q^{\prime}\right)\right\|
$$

for all $q, q^{\prime} \in \operatorname{graph}(\phi)$, i.e. the condition 2. of Proposition 2.15 holds.

We observe that in Euclidean spaces intrinsic Lipschitz maps are the same as Lipschitz maps. The converse is not true (see Example 2.3.9 in [16]) and if $\phi: \mathbf{W} \rightarrow \mathbf{H}$ is intrinsic Lipschitz then this does not yield the existence of a constant $C$ such that

$$
\left\|\phi(a)^{-1} \phi(b)\right\| \leq C\left\|a^{-1} b\right\| \quad \text { for } a, b \in \mathbf{W}
$$

not even locally. In Proposition 3.1.8 in [17] the authors proved that the intrinsic Lipschitz functions, even if non metric Lipschitz, nevertheless are Hölder continuous.

Proposition 2.18. Let $\mathbf{W}, \mathbf{H}$ be complementary subgroups in $\mathbf{G}$ and $\phi: \mathcal{O} \subset$ $\mathbf{W} \rightarrow \mathbf{H}$ be an intrinsic $C_{L}$-Lipschitz function. Then, for all $r>0$,

(1) there is $C_{1}=C_{1}(\phi, r)>0$ such that

$$
\|\phi(a)\| \leq C_{1} \quad \text { for all } a \in \mathcal{O} \text { with }\|a\| \leq r
$$

(2) there is $C_{2}=C_{2}\left(C_{L}, r\right)>0$ such that $\phi$ is locally $1 / \kappa$-Hölder continuous i.e.

$$
\left\|\phi(a)^{-1} \phi(b)\right\| \leq C_{2}\left\|a^{-1} b\right\|^{1 / \kappa} \quad \text { for all } a, b \text { with }\|a\|,\|b\| \leq r
$$

where $\kappa$ is the step of $\mathbf{G}$.

Now we present a result which we will use later:

Proposition 2.19. [11, Proposition 3.6] Let $\mathbf{H}, \mathbf{W}$ be complementary subgroups of $\mathbf{G}$ with $\mathbf{H}$ horizontal. Let $\mathcal{O}$ be open in $\mathbf{W}$ and $\phi: \mathcal{O} \rightarrow \mathbf{H}$ be u.i.d. in $\mathcal{O}$. Then

(1) $\phi$ is intrinsic Lipschitz continuous in every relatively compact subset of $\mathcal{O}$.

(2) the function $a \mapsto d \phi_{a}$ is continuous in $\mathcal{O}$. 
Finally, we recall the following Rademacher type theorem, proved in [16, Theorem 4.3.5].

Theorem 2.20. Let $\mathbf{G}=\mathbf{W} \cdot \mathbf{H}$ be a topologically $N$-dimensional Carnot group of step 2 with $\mathbf{H}$ horizontal and one dimensional and let $\phi: \mathcal{O} \rightarrow \mathbf{H}$ be an intrinsic Lipschitz function, where $\mathcal{O}$ is a relatively open subset of $\mathbf{W}$. Then $\phi$ is intrinsic differentiable $\left(\mathcal{L}^{N-1}\llcorner\mathbf{W})\right.$-a.e. in $\mathcal{O}$. Notice that $\mathcal{L}^{N-1}\llcorner\mathbf{W}$ is the Haar measure of $\mathbf{W}$.

\section{Carnot groups of step 2}

In this section we present Carnot groups of step 2 as in Chapter 3 of [7]. According to (1), the group operation of a Carnot group $\mathbf{G}:=\left(\mathbf{R}^{m+n}, \cdot\right)$ of step 2 is

$$
p \cdot q=\left(p^{1}+q^{1}, p^{2}+q^{2}+\mathcal{Q}^{2}\left(p^{1}, q^{1}\right)\right)
$$

for every $p=\left(p^{1}, p^{2}\right), q=\left(q^{1}, q^{2}\right) \in \mathbf{G}$, where $\mathcal{Q}^{2}=\left(\mathcal{Q}_{1}, \ldots, \mathcal{Q}_{n}\right): \mathbf{R}^{m} \times \mathbf{R}^{m} \rightarrow \mathbf{R}^{n}$ and by Proposition 2.2.22 (4) in [7] we have

$$
\mathcal{Q}_{s}\left(p^{1}, q^{1}\right)=\sum_{j, l=1}^{m} b_{j l}^{s}\left(p_{j} q_{l}-p_{l} q_{j}\right)=\frac{1}{2}\left\langle\mathcal{B}^{(s)} p^{1}, q^{1}\right\rangle, \quad \text { for } s=1, \ldots, n,
$$

where $\mathcal{B}^{(s)}$ is skew-symmetric $m \times m$ real matrix and $\mathcal{B}^{(s)}=\left(b_{j l}^{(s)}\right)_{j, l=1}^{m}$. For any $n$ linearly independent, skew-symmetric $m \times m$ real matrices $\mathcal{B}^{(1)}, \ldots, \mathcal{B}^{(n)}$ such that for all $p=\left(p^{1}, p^{2}\right)$ and $q=\left(q^{1}, q^{2}\right) \in \mathbf{R}^{m} \times \mathbf{R}^{n}$ and for all $\lambda>0$

$$
p \cdot q=\left(p^{1}+q^{1}, p^{2}+q^{2}+\frac{1}{2}\left\langle\mathcal{B} p^{1}, q^{1}\right\rangle\right),
$$

where $\left\langle\mathcal{B} p^{1}, q^{1}\right\rangle:=\left(\left\langle\mathcal{B}^{(1)} p^{1}, q^{1}\right\rangle, \ldots,\left\langle\mathcal{B}^{(n)} p^{1}, q^{1}\right\rangle\right)$ and $\langle\cdot, \cdot\rangle$ is the inner product in $\mathbf{R}^{m}$ and

$$
\delta_{\lambda} p:=\left(\lambda p^{1}, \lambda^{2} p^{2}\right)
$$

the group $\mathbf{G}:=\left(\mathbf{R}^{m+n}, \cdot, \delta_{\lambda}\right)$ is a Carnot group of step 2 with $\mathbf{R}^{m}$ the horizontal layer and $\mathbf{R}^{n}$ the vertical layer (see Proposition 3.2.1 and Theorem 3.2.2 in [7]).

We make the following choice of the homogeneous norm in $\mathbf{G}$ :

$$
\left\|\left(p^{1}, p^{2}\right)\right\|:=\max \left\{\left|p^{1}\right|_{\mathbf{R}^{m}}, \epsilon\left|p^{2}\right|_{\mathbf{R}^{n}}^{1 / 2}\right\}
$$

for a suitable $\epsilon \in(0,1]$ (for the existence of such an $\epsilon>0$ see Theorem 5.1 in [20]). We recall also that $p^{-1}=\left(-p^{1},-p^{2}\right)$ (see (2)) and there is $c_{1}>1$ such that for all $p=\left(p^{1}, p^{2}\right) \in \mathbf{G}$

$$
c_{1}^{-1}\left(\left|p^{1}\right|_{\mathbf{R}^{m}}+\left|p^{2}\right|_{\mathbf{R}^{n}}^{1 / 2}\right) \leq\|p\| \leq c_{1}\left(\left|p^{1}\right|_{\mathbf{R}^{m}}+\left|p^{2}\right|_{\mathbf{R}^{n}}^{1 / 2}\right) .
$$

From now on we will depart slightly from the notations of the previous sections. Precisely, instead of writing $p=\left(p_{1}, \ldots, p_{m+n}\right)$ we will write

$$
p=\left(x_{1}, \ldots, x_{m}, y_{1}, \ldots, y_{n}\right) .
$$

With this notation, when $\mathcal{B}^{(s)}:=\left(b_{j l}^{(s)}\right)_{j, l=1}^{m}$, a basis of the Lie algebra $\mathfrak{g}$ of $\mathbf{G}$, is given by the $m+n$ left invariant vector fields

$$
X_{j}(p)=\partial_{x_{j}}+\frac{1}{2} \sum_{s=1}^{n} \sum_{l=1}^{m} b_{j l}^{(s)} x_{l} \partial_{y_{s}}, \quad Y_{s}(p)=\partial_{y_{s}}
$$

where $j=1, \ldots, m$, and $s=1, \ldots, n$. 
Remark 3.1. The space of skew-symmetric $m \times m$ matrices has dimension $\frac{m(m-1)}{2}$. Hence in any Carnot group $\mathbf{G}$ of step 2 the dimensions $m$ of the horizontal layer and $n$ of the vertical layer are related by the inequality

$$
n \leq \frac{m(m-1)}{2}
$$

Remark 3.2. Heisenberg groups $\mathbf{H}^{k}=\mathbf{R}^{2 k} \times \mathbf{R}$ are Carnot groups of step 2 and the group law is of the form (10) with

$$
\mathcal{B}^{(1)}=\left(\begin{array}{cc}
0 & \mathcal{I}_{k} \\
-\mathcal{I}_{k} & 0
\end{array}\right)
$$

where $\mathcal{I}_{k}$ is the $k \times k$ identity matrix.

More generally, H-type groups are examples of Carnot groups of step 2 (see Definition 3.6.1 and Remark 3.6.7 in [7]). The composition law is of the form (10) where the matrices $\mathcal{B}^{(1)}, \ldots, \mathcal{B}^{(n)}$ have the following additional properties:

(1) $\mathcal{B}^{(s)}$ is an $m \times m$ orthogonal matrix for all $s=1, \ldots n$,

$(2) \mathcal{B}^{(s)} \mathcal{B}^{(l)}=-\mathcal{B}^{(l)} \mathcal{B}^{(s)}$ for every $s, l=1, \ldots, n$ with $s \neq l$.

Any H-type group is a H-group in the sense of Métivier, or a HM-group in short (see Section 3.7 in [7]), which is also example of Carnot groups of step 2. Here the composition law is of the form (10) with the following additional condition: every non-vanishing linear combination of the matrices $\mathcal{B}^{(s)}$ 's is non-singular.

Another example of Carnot groups of step 2 is provided by the class $\mathbf{F}_{m, 2}$ of free groups of step-2 (see Section 3.3 in [7]). Here $\mathbf{F}_{m, 2}=\mathbf{R}^{m} \times \mathbf{R}^{\frac{m(m-1)}{2}}$ and the composition law (10) is defined by the matrices $\mathcal{B}^{(s)} \equiv \mathcal{B}^{(l, j)}$ where $1 \leq j<l \leq m$ and $\mathcal{B}^{(l, j)}$ has entries -1 in position $(l, j), 1$ in position $(j, l)$ and 0 everywhere else.

Notice that Heisenberg groups are H-type groups while $\mathbf{H}^{1}$ is also a free step- 2 group.

3.1. The intrinsic gradient. Let $\mathbf{G}=\left(\mathbf{R}^{m+n}, \cdot, \delta_{\lambda}\right)$ be a Carnot group of step 2 as above and $\mathbf{W}, \mathbf{V}$ be complementary subgroups in $\mathbf{G}$ with $\mathbf{V}$ horizontal and one dimensional.

Remark 3.3. To keep notations simpler, through all this section we assume, without loss of generality, that the complementary subgroups $\mathbf{W}, \mathbf{V}$ are

$$
\mathbf{V}:=\left\{\left(x_{1}, 0 \ldots, 0\right)\right\}, \quad \mathbf{W}:=\left\{\left(0, x_{2}, \ldots, x_{m+n}\right)\right\} .
$$

This amounts simply to a linear change of variables in the first layer of the algebra $\mathfrak{g}$. If we denote $\mathcal{M}$ a non singular $m \times m$ matrix, the linear change of coordinates associated to $\mathcal{M}$ is

$$
p=\left(p^{1}, p^{2}\right) \mapsto\left(\mathcal{M} p^{1}, p^{2}\right) .
$$

The new composition law $\star$ in $\mathbf{R}^{m+n}$, obtained by writing $\cdot$ in the new coordinates, is

$$
\left(\mathcal{M} p^{1}, p^{2}\right) \star\left(\mathcal{M} q^{1}, q^{2}\right):=\left(\mathcal{M} p^{1}+\mathcal{M} q^{1}, p^{2}+q^{2}+\frac{1}{2}\left\langle\tilde{\mathcal{B}} \mathcal{M} p^{1}, \mathcal{M} q^{1}\right\rangle\right),
$$

where $\tilde{\mathcal{B}}:=\left(\tilde{\mathcal{B}}^{(1)}, \ldots, \tilde{\mathcal{B}}^{(n)}\right)$ and $\tilde{\mathcal{B}}^{(s)}=\left(\mathcal{M}^{-1}\right)^{T} \mathcal{B}^{(s)} \mathcal{M}^{-1}$ for $s=1, \ldots, n$. It is easy to check that the matrices $\tilde{\mathcal{B}}^{(1)}, \ldots, \tilde{\mathcal{B}}^{(n)}$ are skew-symmetric and that $\left(\mathbf{R}^{m+n}, \star, \delta_{\lambda}\right)$ is a Carnot groups of step 2 isomorphic to $\mathbf{G}=\left(\mathbf{R}^{m+n}, \cdot, \delta_{\lambda}\right)$.

Remark 3.4. Notice that the constants which appear in Lemma 5.4, Proposition 5.5 and Theorem 5.7 depend on the choice $(\mathbf{V}, \mathbf{W})$. Moreover, the constant 
of Lemma 5.4 is the same if we consider $\left(\mathbf{V}^{\prime}, \mathbf{W}^{\prime}\right)$ with $\mathbf{V}^{\prime}$ one dimensional and orthogonal to $\mathbf{W}$.

When $\mathbf{V}$ and $\mathbf{W}$ are defined as in (13) there is a natural inclusion $i: \mathbf{R}^{m+n-1} \rightarrow$ $\mathbf{W}$ such that, for all $\left(x_{2}, \ldots x_{m}, y_{1}, \ldots, y_{n}\right) \in \mathbf{R}^{m+n-1}$,

$$
i\left(\left(x_{2}, \ldots x_{m}, y_{1}, \ldots, y_{n}\right)\right):=\left(0, x_{2}, \ldots x_{m}, y_{1}, \ldots, y_{n}\right) \in \mathbf{W} .
$$

If $\mathcal{O}$ and $\phi$ are respectively an open set in $\mathbf{R}^{m+n-1}$ and a function $\phi: \mathcal{O} \rightarrow \mathbf{R}$ we denote $\hat{\mathcal{O}}:=i(\mathcal{O}) \subset \mathbf{W}$ and $\hat{\phi}: \hat{\mathcal{O}} \rightarrow \mathbf{V}$ the function defined as

$$
\hat{\phi}(i(a)):=(\phi(a), 0, \ldots, 0)
$$

for all $a \in \mathcal{O}$.

From Theorem 2.13, if $\hat{\phi}: \hat{\mathcal{O}} \subset \mathbf{W} \rightarrow \mathbf{V}$ is such that graph $(\hat{\phi})$ is locally a non critical level set of $f \in \mathbf{C}_{\mathbf{G}}^{1}(\mathbf{G}, \mathbf{R})$ with $X_{1} f \neq 0$, then $\hat{\phi}$ is u.i.d. in $\hat{\mathcal{O}}$ and the following representation of the intrinsic gradient $\nabla^{\hat{\phi}} \hat{\phi}$ holds

$$
\nabla^{\hat{\phi}} \hat{\phi}(p)=-\left(\frac{X_{2} f}{X_{1} f}, \ldots, \frac{X_{m} f}{X_{1} f}\right)(p \cdot \hat{\phi}(p))
$$

for all $p \in \hat{\mathcal{O}}$.

In Proposition 3.5 we prove a different explicit expression of $\nabla^{\hat{\phi}} \hat{\phi}$, not involving $f$, but only derivatives of the real valued function $\phi$.

Proposition 3.5. [11, Proposition 5.4] Let $\mathbf{G}:=\left(\mathbf{R}^{m+n}, \cdot, \delta_{\lambda}\right)$ be a Carnot group of step 2 and $\mathbf{V}, \mathbf{W}$ the complementary subgroups defined in (13). Let $\mathcal{U}$ be open in $\mathbf{G}, f \in \mathbf{C}_{\mathbf{G}}^{1}(\mathcal{U}, \mathbf{R})$ with $X_{1} f>0$ and assume that $S:=\{p \in \mathcal{U}: f(p)=0\}$ is non empty. Then

(i) for every $p \in \mathcal{U}$ there exist $\mathcal{U}^{\prime}$ open neighbourhood of $p$ in $\mathbf{G}, \hat{\mathcal{O}}$ open in $\mathbf{W}$ and $\hat{\psi}: \hat{\mathcal{O}} \rightarrow \mathbf{V}$ such that $S \cap \mathcal{U}^{\prime}=\operatorname{graph}(\hat{\psi}) \cap \mathcal{U}^{\prime}$. Moreover $\hat{\psi}$ is u.i.d. in $\hat{\mathcal{O}}$ and the distributional intrinsic gradient in the sense of Definition 3.2 $\left(D_{2}^{\psi} \psi, \ldots, D_{m}^{\psi} \psi\right)$ of the associated function $\psi: \mathcal{O} \rightarrow \mathbf{R}$ is defined as

$$
D_{j}^{\psi} \psi=X_{j} \psi+\psi \sum_{s=1}^{n} b_{j 1}^{s} Y_{s} \psi, \quad \text { for } j=2, \ldots, m .
$$

Moreover, the distributional intrinsic gradient of $\psi$ has a continuous representative, which is $\nabla^{\hat{\psi}} \hat{\psi}$ (continuous because of (15)), i.e.

$$
\nabla^{\hat{\psi}} \hat{\psi}(i(\cdot))=\left(D_{2}^{\psi} \psi(\cdot), \ldots, D_{m}^{\psi} \psi(\cdot)\right)
$$

holds in the sense of distribution.

(ii) The subgraph $\mathcal{E}:=\{p \in \mathcal{U}: f(p)<0\}$ has locally finite $\mathbf{G}$-perimeter in $\mathcal{U}$ and its $\mathbf{G}$-perimeter measure $|\partial \mathcal{E}|_{\mathbf{G}}$ has the integral representation

$$
|\partial \mathcal{E}|_{\mathbf{G}}(\mathcal{F})=\int_{\Phi^{-1}(\mathcal{F})} \sqrt{1+\left|\nabla^{\hat{\psi}} \hat{\psi}\right|_{\mathbf{R}^{m-1}}^{2}} d \mathcal{L}^{m+n-1}
$$

for every Borel set $\mathcal{F} \subset \mathcal{U}$ where $\Phi: \mathcal{O} \rightarrow \mathbf{G}$ is defined as $\Phi(a):=i(a) \cdot \hat{\psi}(i(a))$ for all $a \in \mathcal{O}$.

From Proposition 3.5, if graph $(\phi)$ is a G-regular hypersurface, the intrinsic gradient of $\phi$ takes the explicit form given in (16). This motivates the definitions of the operators intrinsic horizontal gradient and intrinsic derivatives. 
Definition 3.1. Let $\mathcal{O}$ be open in $\mathbf{R}^{m+n-1}, \phi: \mathcal{O} \rightarrow \mathbf{R}$ be continuous in $\mathcal{O}$. The intrinsic derivatives $D_{j}^{\phi}$, for $j=2, \ldots, m$, are the differential operators with continuous coefficients

$$
D_{j}^{\phi}:=\partial_{x_{j}}+\sum_{s=1}^{n}\left(\phi b_{j 1}^{(s)}+\frac{1}{2} \sum_{i=2}^{m} x_{i} b_{j i}^{(s)}\right) \partial_{y_{s}}=X_{j \mid \mathbf{W}}+\phi \sum_{s=1}^{n} b_{j 1}^{(s)} Y_{s \mid \mathbf{W}},
$$

where, in the second line with abuse of notation, we denote with the same symbols $X_{j}$ and $Y_{s}$ the vector fields acting on functions defined in $\mathcal{O}$. If $\hat{\phi}:=(\phi, 0, \ldots, 0): \hat{\mathcal{O}} \rightarrow$ $\mathbf{V}$, we denote intrinsic horizontal gradient $\nabla^{\hat{\phi}}$ the differential operator

$$
\nabla^{\hat{\phi}}:=\left(D_{2}^{\phi}, \ldots, D_{m}^{\phi}\right) .
$$

Remark 3.6. We emphasize that the intrinsic horizontal gradient $\nabla^{\hat{\psi}} \hat{\psi}$ defined after Definition 3.1 and the continuous representative of the distributional intrinsic gradient as in (7) are different; but according to the approximation argument of Proposition 3.5, they are equal in distributional sense.

Definition 3.2. (Distributional solution) Let $\mathcal{O} \subset \mathbf{R}^{m+n-1}$ be open and $w=$ $\left(w_{2}, \ldots, w_{m}\right) \in \mathcal{L}_{\text {loc }}^{\infty}\left(\mathcal{O}, \mathbf{R}^{m-1}\right)$. We say that $\phi \in \mathbf{C}(\mathcal{O}, \mathbf{R})$ is a distributional solution in $\mathcal{O}$ of the non-linear first order PDE's system

$$
\left(D_{2}^{\phi} \phi, \ldots, D_{m}^{\phi} \phi\right)=w
$$

if for every $\zeta \in \mathbf{C}_{c}^{1}(\mathcal{O}, \mathbf{R})$

$$
\int_{\mathcal{O}} \phi\left(X_{j} \zeta+\phi \sum_{s=1}^{n} b_{j 1}^{(s)} Y_{s} \zeta\right) d \mathcal{L}^{m+n-1}=-\int_{\mathcal{O}} w_{j} \zeta d \mathcal{L}^{m+n-1}
$$

for $j=2, \ldots, m$.

Remark 3.7. Let $m>2$ and $\mathcal{O}$ be a connected set. If the vector fields $D_{2}^{\phi}, \ldots, D_{m}^{\phi}$ are smooth we know that it is possible to connect each couple of points $a$ and $b$ in $\mathcal{O}$ with a piecewise continuous integral curve of horizontal vector fields. This means that there is an absolutely continuous curve $\gamma_{h}:\left[t_{1}, t_{2}\right] \rightarrow \mathcal{O}$ from $a$ to $b$ such that $-\infty<t_{1}<t_{2}<+\infty$ and

$$
\dot{\gamma}_{h}(t)=\sum_{j=2}^{m} h_{j}(t) D_{j}^{\phi}\left(\gamma_{h}(t)\right) \quad \text { a.e. } t \in\left(t_{1}, t_{2}\right)
$$

with $h=\left(h_{2}, \ldots, h_{m}\right):\left[t_{1}, t_{2}\right] \rightarrow \mathbf{R}^{m-1}$ a piecewise continuous function. In our case the vector fields $D_{j}^{\phi}$ are only continuous, and consequently the global existence of $\gamma_{h}$ is not sure.

Proposition 3.8. Let $\mathbf{G}:=\left(\mathbf{R}^{m+n}, \cdot, \delta_{\lambda}\right)$ be a Carnot group of step 2 and $\mathbf{V}$, W the complementary subgroups defined in (13). Let $\hat{\phi}: \hat{\mathcal{O}} \rightarrow \mathbf{V}$ be an intrinsic $C_{L^{-}}$ Lipschitz function, where $\mathcal{O}$ is open in $\mathbf{W}$ and $\phi: \mathcal{O} \rightarrow \mathbf{R}$ is the map associated to $\hat{\phi}$ as in (14). If $\gamma:\left[t_{1}, t_{2}\right] \rightarrow \mathcal{O}$ satisfies the condition (18), then $\left[t_{1}, t_{2}\right] \ni t \mapsto \phi(\gamma(t))$ is Lipschitz continuous.

Proof. The proof of this statement is similar to the one of Proposition 3.8 in [9] in the context of Heisenberg groups. For simplicity we define $\hat{\gamma}(t):=i(\gamma(t)) \in \hat{\mathcal{O}}$. We would like to show that there exists $C_{1}>0$ such that

$$
\left\|\hat{\phi}(\hat{\gamma}(t))^{-1} \hat{\gamma}(t)^{-1} \hat{\gamma}\left(t_{1}\right) \hat{\phi}(\hat{\gamma}(t))\right\| \leq C_{1}\left(t-t_{1}\right)
$$


for all $t \in\left[t_{1}, t_{2}\right]$. In fact since $\hat{\phi}$ is an intrinsic $C_{L}$-Lipschitz function and recall Remark 2.16 we have

$$
\left|\phi(\gamma(t))-\phi\left(\gamma\left(t_{1}\right)\right)\right| \leq C_{L}\left\|\hat{\phi}(\hat{\gamma}(t))^{-1} \hat{\gamma}(t)^{-1} \hat{\gamma}\left(t_{1}\right) \hat{\phi}(\hat{\gamma}(t))\right\| \leq C_{L} C_{1}\left(t-t_{1}\right) .
$$

By hypothesis $\gamma=\gamma_{h}:\left[t_{1}, t_{2}\right] \rightarrow \mathcal{O}$ is an absolutely continuous curve satisfying (18) with a piecewise continuous function $h=\left(h_{2}, \ldots, h_{m}\right) \in \mathcal{L}^{\infty}\left(\left(t_{1}, t_{2}\right), \mathbf{R}^{m-1}\right)$ and $\gamma_{h}\left(t_{1}\right)=a=(x, y), \gamma_{h}\left(t_{2}\right)=b=\left(x^{\prime}, y^{\prime}\right)$. More precisely, we have $\gamma_{h}(t)=$ $\left(x_{2}(t), \ldots, x_{m}(t), y_{1}(t), \ldots, y_{n}(t)\right)=\left(x_{\gamma}(t), y_{1}(t), \ldots, y_{n}(t)\right)$ such that

$$
\begin{aligned}
x_{l}(t)-x_{l}= & \int_{t_{1}}^{t} h_{l}(r) d r \quad \text { for all } t \in\left[t_{1}, t_{2}\right], l \in 2, \ldots, m, \\
y_{s}(t)-y_{s}= & \sum_{j=2}^{m}\left(b_{j 1}^{(s)} \int_{t_{1}}^{t} h_{j}(r) \phi\left(\gamma_{h}(r)\right) d r\right. \\
& \left.+\frac{1}{2} \sum_{l=2}^{m} b_{j l}^{(s)}\left(x_{l}+\int_{t_{1}}^{r} h_{l}\left(r^{\prime}\right) d r^{\prime}\right) \int_{t_{1}}^{t} h_{j}(r) d r\right)
\end{aligned}
$$

for all $t \in\left(t_{1}, t_{2}\right), s=1, \ldots, n$.

Now we consider

$$
\sigma_{\phi}(b, a):=\sum_{s=1}^{n}\left|y_{s}-y_{s}^{\prime}+\phi(b)\left(\sum_{l=2}^{m}\left(x_{l}-x_{l}^{\prime}\right) b_{1 l}^{(s)}\right)-\frac{1}{2}\left\langle\mathcal{B}^{(s)} x^{\prime}, x\right\rangle\right|^{1 / 2} .
$$

If we put $\|\mathcal{B}\|_{\infty}=\max \left\{b_{i j}^{(s)} \mid i, j=1, \ldots, m, s=1, \ldots, n\right\}$, then it easy to see that

$$
\sigma_{\phi}(b, a) \leq \sigma_{\phi}(a, b)+n \sqrt{\|\mathcal{B}\|_{\infty}}|\phi(a)-\phi(b)|^{1 / 2}\left|x-x^{\prime}\right|_{\mathbf{R}^{m-1}}^{1 / 2}
$$

and, recalling (11), if $b=\gamma_{h}(t)$, then

$$
\begin{aligned}
\left\|\hat{\phi}(\hat{\gamma}(t))^{-1} \hat{\gamma}(t)^{-1} \hat{\gamma}\left(t_{1}\right) \hat{\phi}(\hat{\gamma}(t))\right\| \leq & c_{1}\left(\left|x-x_{\gamma}(t)\right|_{\mathbf{R}^{m-1}}+\sigma_{\phi}\left(a, \gamma_{h}(t)\right)\right. \\
& \left.+n \sqrt{\|\mathcal{B}\|_{\infty}}\left|x-x_{\gamma}(t)\right|_{\mathbf{R}^{m-1}}^{1 / 2}\left|\phi\left(\gamma_{h}(t)\right)-\phi(a)\right|^{1 / 2}\right) .
\end{aligned}
$$

Since $\hat{\phi}$ is intrinsic $C_{L}$-Lipschitz, it follows

$$
\begin{aligned}
& n \sqrt{\|\mathcal{B}\|_{\infty}}\left|x-x_{\gamma}(t)\right|_{\mathbf{R}^{m-1}}^{1 / 2}\left|\phi\left(\gamma_{h}(t)\right)-\phi(a)\right|^{1 / 2} \\
& \leq n \sqrt{C_{L}\|\mathcal{B}\|_{\infty}}\left|x-x_{\gamma}(t)\right|_{\mathbf{R}^{m-1}}^{1 / 2}\left\|\hat{\phi}(\hat{\gamma}(t))^{-1} \hat{\gamma}(t)^{-1} \hat{\gamma}\left(t_{1}\right) \hat{\phi}(\hat{\gamma}(t))\right\|^{1 / 2} \\
& \leq \frac{n^{2} C_{L}\|\mathcal{B}\|_{\infty}}{2}\left|x-x_{\gamma}(t)\right|_{\mathbf{R}^{m-1}}+\frac{1}{2}\left\|\hat{\phi}(\hat{\gamma}(t))^{-1} \hat{\gamma}(t)^{-1} \hat{\gamma}\left(t_{1}\right) \hat{\phi}(\hat{\gamma}(t))\right\|
\end{aligned}
$$

for all $t \in\left[t_{1}, t_{2}\right]$. From $(20)$

$$
\left|x-x_{\gamma}(t)\right|_{\mathbf{R}^{m-1}} \leq(m-1)\left(t-t_{1}\right)\|h\|_{\mathcal{L}^{\infty}\left(\left(t_{1}, t_{2}\right), \mathbf{R}^{m-1}\right)}
$$

and, consequently, if we put $M_{1}:=c_{1}(m-1)\left(2+C_{L}\|\mathcal{B}\|_{\infty} n^{2}\right)$, then

(24) $\left\|\hat{\phi}(\hat{\gamma}(t))^{-1} \hat{\gamma}(t)^{-1} \hat{\gamma}\left(t_{1}\right) \hat{\phi}(\hat{\gamma}(t))\right\| \leq 2 c_{1} \sigma_{\phi}\left(a, \gamma_{h}(t)\right)+M_{1}\left(t-t_{1}\right)\|h\|_{\mathcal{L}^{\infty}\left(\left(t_{1}, t_{2}\right), \mathbf{R}^{m-1}\right)}$. 
Hence it remains to estimate $\sigma_{\phi}\left(a, \gamma_{h}(t)\right)$. By (20) and (21) we observe that

$$
\begin{aligned}
\sigma_{\phi}\left(a, \gamma_{h}(t)\right)= & \sum_{s=1}^{n} \mid \sum_{j=2}^{m}\left(b_{j 1}^{(s)} \int_{t_{1}}^{t} h_{j}(r) \phi\left(\gamma_{h}(r)\right) d r\right. \\
& \left.+\frac{1}{2} \sum_{l=2}^{m} b_{j l}^{(s)}\left(x_{l}+\int_{t_{1}}^{r} h_{l}\left(r^{\prime}\right) d r^{\prime}\right) \int_{t_{1}}^{t} h_{j}(r) d r\right) \\
& +\phi(a) \sum_{l=2}^{m} b_{1 l}^{(s)} \int_{t_{1}}^{t} h_{l}(r) d r-\left.\frac{1}{2}\left\langle\mathcal{B}^{(s)} x, x_{\gamma}(t)\right\rangle\right|^{1 / 2}
\end{aligned}
$$

with

$\sum_{l, j=2}^{m} x_{l} b_{j l}^{(s)} \int_{t_{1}}^{t} h_{j}(r) d r-\left\langle\mathcal{B}^{(s)} x, x_{\gamma}(t)\right\rangle=\sum_{l, j=2}^{m} x_{l} b_{j l}^{(s)}\left(x_{j}(t)-x_{j}\right)-\left\langle\mathcal{B}^{(s)} x, x_{\gamma}(t)-x\right\rangle=0$

and

$$
\left|\frac{1}{2} \sum_{l, j=2}^{m} b_{j l}^{(s)} \int_{t_{1}}^{r} h_{l}\left(r^{\prime}\right) d r^{\prime} \int_{t_{1}}^{t} h_{j}(r) d r\right| \leq \frac{1}{2}\|\mathcal{B}\|_{\infty}(m-1)^{2}\left(t-t_{1}\right)^{2}\|h\|_{\mathcal{L}^{\infty}\left(\left(t_{1}, t_{2}\right), \mathbf{R}^{m-1}\right)}^{2}
$$

Hence, remembering that $\phi$ is intrinsic $C_{L}$-Lipschitz function, it follows

$$
\begin{aligned}
\sigma_{\phi}\left(a, \gamma_{h}(t)\right) \leq & \sum_{s=1}^{n}\left|\sum_{j=2}^{m} b_{j 1}^{(s)} \int_{t_{1}}^{t} h_{j}(r)\left(\phi(a)-\phi\left(\gamma_{h}(r)\right)\right) d r\right|^{1 / 2} \\
& +\frac{\sqrt{2\|\mathcal{B}\|_{\infty}}}{2} n(m-1)\left(t-t_{1}\right)\|h\|_{\mathcal{L}^{\infty}\left(\left(t_{1}, t_{2}\right), \mathbf{R}^{m-1}\right)} \\
\leq & n \sqrt{\|\mathcal{B}\|_{\infty} C_{L}(m-1)\left(t-t_{1}\right)}\|h\|_{\mathcal{L}^{\infty}\left(\left(t_{1}, t_{2}\right), \mathbf{R}^{m-1}\right)}^{1 / 2} \\
& \cdot \max _{r \in[a, b]}\left\|\hat{\phi}(\hat{\gamma}(t))^{-1} \hat{\gamma}(t)^{-1} \hat{\gamma}\left(t_{1}\right) \hat{\phi}(\hat{\gamma}(t))\right\|^{1 / 2} \\
& +\frac{\sqrt{2\|\mathcal{B}\|_{\infty}}}{2} n(m-1)\left(t-t_{1}\right)\|h\|_{\mathcal{L}^{\infty}\left(\left(t_{1}, t_{2}\right), \mathbf{R}^{m-1}\right)}
\end{aligned}
$$

Since (24) holds, we conclude that

$$
\begin{aligned}
& \max _{t \in\left[t_{1}, t_{2}\right]}\left\|\hat{\phi}(\hat{\gamma}(t))^{-1} \hat{\gamma}(t)^{-1} \hat{\gamma}\left(t_{1}\right) \hat{\phi}(\hat{\gamma}(t))\right\| \leq M_{1}\left(t-t_{1}\right)\|h\|_{\mathcal{L}^{\infty}\left(\left(t_{1}, t_{2}\right), \mathbf{R}^{m-1}\right)} \\
& \quad+2 c_{1} n \sqrt{\|\mathcal{B}\|_{\infty} C_{L}(m-1)\left(t-t_{1}\right)}\|h\|_{\mathcal{L}^{\infty}\left(\left(t_{1}, t_{2}\right), \mathbf{R}^{m-1}\right)}^{1 / 2} \\
& \quad \cdot \max _{t \in\left[t_{1}, t_{2}\right]}\left\|\hat{\phi}(\hat{\gamma}(t))^{-1} \hat{\gamma}(t)^{-1} \hat{\gamma}\left(t_{1}\right) \hat{\phi}(\hat{\gamma}(t))\right\|^{1 / 2} \\
& \quad+\sqrt{2\|\mathcal{B}\|_{\infty}} c_{1} n(m-1)\left(t-t_{1}\right)\|h\|_{\mathcal{L}^{\infty}\left(\left(t_{1}, t_{2}\right), \mathbf{R}^{m-1}\right)} \\
& \leq\left(M_{1}+8 c_{1}^{2} n^{2}(m-1)\|\mathcal{B}\|_{\infty} C_{L}+\sqrt{2\|\mathcal{B}\|_{\infty}} c_{1} n(m-1)\right) \\
& \quad \cdot(t-a)\|h\|_{\mathcal{L}^{\infty}\left(\left(t_{1}, t_{2}\right), \mathbf{R}^{m-1}\right)}+\frac{1}{2} \max _{t \in\left[t_{1}, t_{2}\right]}\left\|\hat{\phi}(\hat{\gamma}(t))^{-1} \hat{\gamma}(t)^{-1} \hat{\gamma}\left(t_{1}\right) \hat{\phi}(\hat{\gamma}(t))\right\| \\
&=: \frac{M_{2}}{2}\left(t-t_{1}\right)\|h\|_{\mathcal{L}^{\infty}\left(\left(t_{1}, t_{2}\right), \mathbf{R}^{m-1}\right)}+\frac{1}{2} \max _{t \in\left[t_{1}, t_{2}\right]}\left\|\hat{\phi}(\hat{\gamma}(t))^{-1} \hat{\gamma}(t)^{-1} \hat{\gamma}(a) \hat{\phi}(\hat{\gamma}(t))\right\| .
\end{aligned}
$$

Consequently for all $t \in\left[t_{1}, t_{2}\right]$

$$
\left\|\hat{\phi}(\hat{\gamma}(t))^{-1} \hat{\gamma}(t)^{-1} \hat{\gamma}\left(t_{1}\right) \hat{\phi}(\hat{\gamma}(t))\right\| \leq M_{2}\left(t-t_{1}\right)\|h\|_{\mathcal{L}^{\infty}\left(\left(t_{1}, t_{2}\right), \mathbf{R}^{m-1}\right)}=: C_{1}\left(t-t_{1}\right)
$$


i.e. (19) holds.

Remark 3.9. Notice that in Theorem 4.2.16 in [28], it is proved, in general setting of Carnot groups, the statement of Proposition 3.8 but just for horizontal curves $\gamma:\left[t_{1}, t_{2}\right] \rightarrow \mathcal{O}$ with constant controls $h_{j}(t) \equiv h_{j}$ in $(18)$.

\section{Caccioppoli sets}

Let $\mathbf{G}$ be a Carnot group of step 2. Let $\mathbf{S}^{m-1}$ be the unit sphere of $\mathbf{R}^{m}$ and $\nu \in \mathbf{S}^{m-1}$, i.e. $\nu \in \mathbf{R}^{m}$ and $|\nu|_{\mathbf{R}^{m}}=1$. By abuse of notation we identify $\nu=$ $\left(\nu_{1}, \ldots, \nu_{m}\right) \in \mathbf{R}^{m}$ and $\nu=(\nu, 0, \ldots, 0) \in \mathbf{G}$.

Fix $p=\left(p^{1}, p^{2}\right) \in \mathbf{G}$ with $p^{1} \in \mathbf{R}^{m}, p^{2} \in \mathbf{R}^{n}$. Let $\nu(p)=\langle p, \nu\rangle \nu \in \mathbf{G}$ and we define $\nu^{\perp}(p) \in \mathbf{G}$ as the unique point such that

$$
p=\nu^{\perp}(p) \cdot \nu(p)
$$

More precisely,

$$
\nu^{\perp}(p)=\left(p^{1}-\left\langle p^{1}, \nu\right\rangle \nu, p^{2}-\frac{1}{2}\left\langle p^{1}, \nu\right\rangle\left\langle\mathcal{B} p^{1}, \nu\right\rangle\right) .
$$

We denote by $\nu^{\perp}=\left\{p=\left(p^{1}, p^{2}\right) \in \mathbf{G}:\left\langle p^{1}, \nu\right\rangle=0\right\}$ the orthogonal complement of $\nu$ in $\mathbf{G}$. It is clear that $\nu^{\perp}(p) \in \nu^{\perp}$ for every $p \in \mathbf{G}$. We observe that if $\nu=(1,0, \ldots, 0)$, then

$$
\nu^{\perp}(p)=p_{\mathbf{W}}, \quad \nu(p)=p_{\mathbf{V}},
$$

where $\mathbf{W}, \mathbf{V}$ are the complementary subgroups defined as (13). Precisely, for $\nu \in$ $\mathbf{S}^{m-1}$ we have $\mathbf{V}_{\nu}=\{(t \nu, 0 \ldots, 0): t \in \mathbf{R}\}$ and $\mathbf{W}_{\nu}=\nu^{\perp} \times \mathbf{R}^{n}$. Moreover according to Definition 2.10, the set

$$
\left\{q \in \mathbf{G}:\left\|\nu^{\perp}\left(p^{-1} q\right)\right\| \leq \beta\left\|\nu\left(p^{-1} q\right)\right\|\right\}
$$

is an intrinsic cone with vertex $p$, opening $\beta>0$ and axis specified by $\nu$.

The main results of this section are Theorem 4.1 and Theorem 4.2. We show that the boundary of set with finite $\mathbf{G}$-perimeter and having a bound on the orientation of the measure theoretic normal is an intrinsic graph of an intrinsic Lipschitz function.

The proof of Theorem 4.1 is based on the following observation: if we start from a point of $E \cap \partial \mathcal{U}(0, r)$ with positive lower density and we move for a short time along a horizontal direction near $\nu$, then we remain in the set of positive lower density of $E$. We can then show that for each point of $E \cap \partial \mathcal{U}(0, r)$ there is a truncated lateral cone with fixed opening that is contained in $E$. We use a similar technique exploited in Theorem 1.1 in [34] in the context of Heisenberg groups.

Theorem 4.1 will later be applied in Section 6 to prove the smooth approximation theorem for intrinsic Lipschitz maps (see Theorem 6.1).

Theorem 4.1. Let $\mathbf{G}$ be a Carnot group of step 2 and let $E \subset \mathbf{G}$ be a set with finite $\mathbf{G}$-perimeter in $\mathcal{U}(0, r), \nu_{E}$ be the measure theoretic inward normal of $E$ and $\nu \in \mathbf{S}^{m-1}$. Assume that there exists $k \in(0,1)$ such that $\left\langle\nu_{E}(p), \nu\right\rangle \leq-k$ for $|\partial E|_{\mathbf{G}^{-}}$-a.e. $p \in \mathcal{U}(0, r)$. Then there exists $\beta>0$ such that possibly modifying $E$ in a negligible set, we get for every $p \in \partial E \cap \mathcal{U}(0, r)$

$$
\begin{aligned}
& \left\{q \in \mathcal{U}(0, r):\left\|\nu^{\perp}\left(p^{-1} q\right)\right\|<-\beta\left\langle p^{-1} q, \nu\right\rangle\right\} \subset E, \\
& \left\{q \in \mathcal{U}(0, r):\left\|\nu^{\perp}\left(p^{-1} q\right)\right\|<\beta\left\langle p^{-1} q, \nu\right\rangle\right\} \subset \mathbf{G}-E .
\end{aligned}
$$


Theorem 4.2. Under the same assumptions of Theorem 4.1, possibly modifying $E$ on a negligible set, the set $\partial E \cap \mathcal{U}(0, r)$ is the intrinsic graph of an intrinsic Lipschitz function.

Firstly we prove some preliminary results.

Lemma 4.3. Let $\mathbf{G}$ be a Carnot group of step 2 and let $E \subset \mathbf{G}$ be a locally finite $\mathbf{G}$-perimeter set. Then for all $\mathcal{U}(p, r)$ with $p \in \mathbf{G}$ and $r>0$, if we consider a horizontal left invariant vector field $Z$ satisfying

$$
\int_{E} Z \xi d \mathcal{L}^{m+n} \leq 0 \quad \forall \xi \in \mathbf{C}_{c}^{1}(\mathcal{U}(p, r), \mathbf{R}), \quad \xi \geq 0
$$

for each $\mathcal{L}^{m+n}$-measurable set $F \subset \mathcal{U}(p, r)$, we obtain

$$
\mathcal{L}^{m+n}(E \cap F) \leq \mathcal{L}^{m+n}(E \cap \exp t Z(F))
$$

for all $t \geq 0$ such that $\exp t Z(F) \subset \mathcal{U}(p, r)$.

Proof. We use the similar technique exploited in Lemma 2.1 in [34] in the context of Heisenberg groups. Let $\mathcal{U}(0, r)$ with $r>0$. Notice that because the homogeneous norm is invariant we can assume $p=0$. Moreover thanks to Remark 3.3, without loss of generality, we also assume $Z=X_{1}$.

We consider the map $\Theta: \mathbf{G} \rightarrow \mathbf{G}$ given by

$$
\Theta(p)=\exp p_{1} X_{1}\left(0, p_{2}, \ldots, p_{m+n}\right) .
$$

It is a global diffeomorphism and it satisfies

$$
\operatorname{det} d \Theta(p)=1 \quad \text { and } \quad \Theta_{*}\left(\partial_{p_{1}}\right)=X_{1} .
$$

where $d \Theta$ denotes the differential of $\Theta$. If we put $E_{1}:=\Theta^{-1}(E)$ and $F_{1}:=\Theta^{-1}(F)$, then

$$
\Theta\left(t e_{1}+F_{1}\right)=\exp \left(t X_{1}(F)\right), \quad t \in \mathbf{R},
$$

with $e_{1}=(1,0, \ldots, 0) \in \mathbf{R}^{m+n}$. Moreover for all $\theta \in \mathbf{C}_{c}^{1}\left(\Theta^{-1}(\mathcal{U}(0, r)), \mathbf{R}\right)$ with $\theta \geq 0$ we define $\xi(q):=\theta\left(\Theta^{-1}(q)\right)$ and consequently by $(29)$ and $(30)$

$$
\int_{E_{1}} \partial_{p_{1}} \theta(p) d \mathcal{L}^{m+n}(p)=\int_{E} X_{1} \xi(q) d \mathcal{L}^{m+n}(q) \leq 0 .
$$

Hence by Fubini-Tonelli Theorem and by a standard approximation argument we know that the function $t \mapsto \chi_{E_{1}}\left(p+t e_{1}\right)$ is increasing for $\mathcal{L}^{m+n}$-a.e. $p \in \Theta^{-1}(\mathcal{U}(0, r))$ as long as $p+t e_{1} \in \Theta^{-1}(\mathcal{U}(0, r))$. Then for a certain $t \geq 0$, using again Fubini-Tonelli Theorem we obtain

$$
\begin{aligned}
\mathcal{L}^{m+n}\left(E_{1} \cap F_{1}\right) & =\int_{\mathbf{R}^{m+n-1}} \int_{\mathbf{R}} \chi_{E_{1}}(p) \chi_{F_{1}}(p) d \mathcal{L} d \mathcal{L}^{m+n-1} \\
& \leq \int_{\mathbf{R}^{m+n-1}} \int_{\mathbf{R}} \chi_{E_{1}}\left(t e_{1}+p\right) \chi_{F_{1}}(p) d \mathcal{L} d \mathcal{L}^{m+n-1} \\
& =\int_{\mathbf{R}^{m+n-1}} \int_{\mathbf{R}} \chi_{E_{1}}(p) \chi_{t e_{1}+F_{1}}(p) d \mathcal{L} d \mathcal{L}^{m+n-1} \\
& =\mathcal{L}^{m+n}\left(E_{1} \cap\left(t e_{1}+F_{1}\right)\right) .
\end{aligned}
$$

Finally by the last inequality, (30) and (31) we obtain

$$
\mathcal{L}^{m+n}(E \cap F) \leq \mathcal{L}^{m+n}\left(E \cap \exp \left(t X_{1}(F)\right)\right) .
$$

Consequently the proof is complete. 
Lemma 4.4. Let $\mathbf{G}$ be a Carnot group of step 2. If $k \in(0,1]$, then there exists $\beta=\beta(k)>0$ such that for all $\nu \in \mathbf{S}^{m-1}$ and $p=\left(p^{1}, p^{2}\right) \in \mathbf{G}$ satisfying

$$
\left\|\nu^{\perp}(p)\right\|=\max \left\{\left|p^{1}-\left\langle p^{1}, \nu\right\rangle \nu\right|_{\mathbf{R}^{m}}, \epsilon\left|p^{2}-\frac{1}{2}\left\langle p^{1}, \nu\right\rangle\left\langle\mathcal{B} p^{1}, \nu\right\rangle\right|_{\mathbf{R}^{n}}^{1 / 2}\right\} \leq-\beta\left\langle p^{1}, \nu\right\rangle,
$$

there exist $\eta_{1}, \ldots, \eta_{n} \in \mathbf{R}^{m}$ such that for all $s=1, \ldots, n$,

$$
\begin{aligned}
\left\langle\eta_{s}, \nu\right\rangle & \leq-\frac{\sqrt{1-k^{2}}\left|\eta_{s}\right|_{\mathbf{R}^{m}}}{1+\|\mathcal{B}\|_{\infty}}\left\langle p^{1}-\eta_{s}, \nu\right\rangle \leq-\frac{\sqrt{1-k^{2}}\left|p^{1}-\eta_{s}\right|_{\mathbf{R}^{m}}}{1+\|\mathcal{B}\|_{\infty}} \\
p^{2} & =\left(\left\langle\mathcal{B}^{(1)} \eta_{1}, p^{1}\right\rangle, \ldots,\left\langle\mathcal{B}^{(n)} \eta_{n}, p^{1}\right\rangle\right)
\end{aligned}
$$

where $\|\mathcal{B}\|_{\infty}=\max \left\{b_{j l}^{(s)}: s=1, \ldots n\right.$ and $\left.j, l=1, \ldots, m\right\}$.

Proof. We split the proof of this lemma in several steps.

Step 1. If $m=2$ and $n=1$, then the thesis follows from Step 1 of the proof of Proposition 2.2 in [34] with the constant $b_{12} \neq 0$ instead of 1 . Indeed, running the computation, in our case, we have

$$
\mathcal{B}^{(1)}=\left(\begin{array}{cc}
0 & b_{12} \\
-b_{12} & 0
\end{array}\right)
$$

with $b_{12} \neq 0$ and $\beta>0$ is a number satisfying

$$
\begin{aligned}
\beta\left(\frac{\beta}{\epsilon^{2}}-\frac{b_{12}}{2}\right) & \leq \frac{3 b_{12} h}{8} \text { with } h=\sqrt{\frac{k^{2}}{2-k^{2}}}, \\
\beta^{2} & \leq \frac{k^{2}}{2-2 k^{2}} .
\end{aligned}
$$

Step 2. We generalize the statement from $m=2$ to arbitrary $m$. Here $n=1$. Let $\left(p^{1}, p^{2}\right) \in \mathbf{R}^{m} \times \mathbf{R}$ be a point satisfying (32) relatively to $\nu$. We denote $\mathcal{A}=$ $\operatorname{span}\left\{p^{1}, \mathcal{B}^{(1)} p^{1}\right\}$ and we consider the orthogonal projection of $\nu$ onto $\mathcal{A}$ by

$$
\pi_{\mathcal{A}} \nu=\frac{\left(\left\langle p^{1}, \nu\right\rangle+\left\langle\mathcal{B}^{(1)} p^{1}, \nu\right\rangle\right)\left(p^{1}+\mathcal{B}^{(1)} p^{1}\right)}{\left|p^{1}+\mathcal{B}^{(1)} p^{1}\right|_{\mathbf{R}^{m}}^{2}} .
$$

If we put

$$
\hat{\nu}=\frac{\pi_{\mathcal{A}} \nu}{\left|\pi_{\mathcal{A}} \nu\right|} \quad \text { and } \quad \xi=\left|\pi_{\mathcal{A}} \nu\right|
$$

then by (32) we conclude that

$$
\frac{1}{\left(1+\|\mathcal{B}\|_{\infty}\right) \sqrt{1+\beta^{2}}} \leq \xi \leq 1,
$$

where $\|\mathcal{B}\|_{\infty}=\max \left\{b_{j l}: j, l=1, \ldots, m\right\}$. Now we observe that if $\left(p^{1}, p^{2}\right)$ satisfies (32) relatively to $\nu$, then $\left(p^{1}, \hat{p}^{2}\right)$ with $\hat{p}^{2}=p^{2} / \xi^{2}$ satisfies (32) relatively to $\hat{\nu}$ with the same $\beta$, indeed

$$
\begin{aligned}
& \epsilon\left|p^{2}-\frac{1}{2}\left\langle p^{1}, \nu\right\rangle\left\langle\mathcal{B}^{(1)} p^{1}, \nu\right\rangle\right|^{1 / 2} \leq-\beta\left\langle p^{1}, \nu\right\rangle \\
& \Longleftrightarrow \quad \epsilon\left|\hat{p}^{2}-\frac{1}{2}\left\langle p^{1}, \hat{\nu}\right\rangle\left\langle\mathcal{B}^{(1)} p^{1}, \hat{\nu}\right\rangle\right|^{1 / 2} \leq-\beta\left\langle p^{1}, \hat{\nu}\right\rangle .
\end{aligned}
$$

Moreover, if $\left|p^{1}\right|_{\mathbf{R}^{m}} \leq-\sqrt{1+\beta^{2}}\left\langle p^{1}, \nu\right\rangle$, then

$$
\left|p^{1}\right|_{\mathbf{R}^{m}} \leq-\xi \sqrt{1+\beta^{2}}\left\langle p^{1}, \hat{\nu}\right\rangle \leq-\sqrt{1+\beta^{2}}\left\langle p^{1}, \hat{\nu}\right\rangle
$$


and consequently $\left|p^{1}-\left\langle p^{1}, \hat{\nu}\right\rangle \hat{\nu}\right|_{\mathbf{R}^{m}} \leq-\beta\left\langle p^{1}, \hat{\nu}\right\rangle$. By Step 1 we know that there exists $\hat{\eta} \in \mathcal{A}$ such that $\hat{p}^{2}=\left\langle\mathcal{B}^{(1)} \hat{\eta}, p^{1}\right\rangle$, where $\eta=\xi^{2} \hat{\eta}$ solves $p^{2}=\left\langle\mathcal{B}^{(1)} \eta, p^{1}\right\rangle$. Moreover,

$$
\langle\hat{\eta}, \hat{\nu}\rangle \leq-\sqrt{1-\frac{k^{2}}{2}}|\hat{\eta}|_{\mathbf{R}^{m}} \quad \text { and } \quad\left\langle p^{1}-\hat{\eta}, \hat{\nu}\right\rangle \leq-\sqrt{1-\frac{k^{2}}{2}}\left|p^{1}-\hat{\eta}\right|_{\mathbf{R}^{m}}
$$

Taking into account (35), (36) and (39) we get the first inequality in (33), indeed

$$
\begin{aligned}
\langle\eta, \nu\rangle & =\xi\langle\eta, \hat{\nu}\rangle \leq \xi^{3}\langle\hat{\eta}, \hat{\nu}\rangle \leq-\xi|\eta|_{\mathbf{R}^{m}} \sqrt{1-\frac{k^{2}}{2}} \\
& \leq-|\eta|_{\mathbf{R}^{m}} \frac{\sqrt{1-\frac{k^{2}}{2}}}{\left(1+\|\mathcal{B}\|_{\infty}\right) \sqrt{1+\beta^{2}}} \leq-\frac{|\eta|_{\mathbf{R}^{m}} \sqrt{1-k^{2}}}{1+\|\mathcal{B}\|_{\infty}} .
\end{aligned}
$$

Finally, using (39)

$$
\begin{aligned}
\left\langle p^{1}-\eta, \nu\right\rangle & =\left\langle p^{1}-\xi^{2} \hat{\eta}, \nu\right\rangle=\xi^{2}\left\langle p^{1}-\hat{\eta}, \nu\right\rangle+\left(1-\xi^{2}\right)\left\langle p^{1}, \nu\right\rangle \\
& =\xi^{3}\left\langle p^{1}-\hat{\eta}, \hat{\nu}\right\rangle+\xi\left(1-\xi^{2}\right)\left\langle p^{1}, \hat{\nu}\right\rangle \\
& \leq \xi^{3}\left(-\sqrt{1-\frac{k^{2}}{2}}\left|p^{1}-\hat{\eta}\right|_{\mathbf{R}^{m}}\right)+\xi\left(1-\xi^{2}\right)\left\langle p^{1}, \hat{\nu}\right\rangle
\end{aligned}
$$

and by (36) and (38)

$$
\begin{aligned}
\left\langle p^{1}-\eta, \nu\right\rangle & \leq \xi\left(-\sqrt{1-\frac{k^{2}}{2}}\left|\xi^{2} p^{1}-\eta\right|_{\mathbf{R}^{m}}\right)+\left(1-\xi^{2}\right) \frac{-\left|p^{1}\right|_{\mathbf{R}^{m}}}{\left(1+\|\mathcal{B}\|_{\infty}\right) \sqrt{1+\beta^{2}}} \\
& \leq-\frac{\sqrt{1-\frac{k^{2}}{2}}}{\left(1+\|\mathcal{B}\|_{\infty}\right) \sqrt{1+\beta^{2}}}\left|\xi^{2} p^{1}-\eta\right|_{\mathbf{R}^{m}}-\frac{\left|\left(1-\xi^{2}\right) p^{1}\right|_{\mathbf{R}^{m}}}{\left(1+\|\mathcal{B}\|_{\infty}\right) \sqrt{1+\beta^{2}}} \\
& \leq-\frac{\sqrt{1-k^{2}}\left|\xi^{2} p^{1}-\eta\right|_{\mathbf{R}^{m}}-\left|\left(1-\xi^{2}\right) p^{1}\right|_{\mathbf{R}^{m}} \sqrt{1-k^{2}}}{1+\mathcal{B}_{m}} \\
& \leq-\frac{\sqrt{1-k^{2}}\left|p^{1}-\hat{\eta}\right|_{\mathbf{R}^{m}}}{1+\|\mathcal{B}\|_{\infty}}
\end{aligned}
$$

i.e. the second inequality in (33) follows.

Step 3. We generalize the statement from $n=1$ to arbitrary $n$. Here $m$ is a natural number larger than 2. This is the main difference in the case of Heisenberg groups; indeed in Heisenberg groups there is only one vertical coordinate (i.e. $n=1$ ).

Let $\left(p^{1}, p^{2}\right)$ be a point satisfying $(32)$ relatively to $\nu$ with $p^{2}=\left(p_{m+1}, \ldots, p_{m+n}\right)$. By (32), we have that

$$
\epsilon\left|\hat{p}^{2}-\frac{1}{2}\left\langle p^{1}, \hat{\nu}\right\rangle\left\langle\mathcal{B} p^{1}, \hat{\nu}\right\rangle\right|_{\mathbf{R}^{n}}^{1 / 2} \leq-\beta\left\langle p^{1}, \hat{\nu}\right\rangle
$$

and this implies

$$
\epsilon\left|\hat{p}_{m+s}-\frac{1}{2}\left\langle p^{1}, \hat{\nu}\right\rangle\left\langle\mathcal{B}^{(s)} p^{1}, \hat{\nu}\right\rangle\right|^{1 / 2} \leq-\beta\left\langle p^{1}, \hat{\nu}\right\rangle
$$

for all $s=1, \ldots n$. Consequently, the point $\left(p^{1}, \hat{p}_{m+s}\right) \in \mathbf{R}^{m} \times \mathbf{R}$ with $\hat{p}_{m+s}=p_{m+s} / \xi^{2}$ satisfies (32) relatively to $\hat{\nu}$ with the same $\beta$. By Step 2 there exists $\eta_{s} \in \mathbf{R}^{m}$ such that

$$
\left\langle\eta_{s}, \nu\right\rangle \leq-\frac{1}{1+\|\mathcal{B}\|_{\infty}} \sqrt{1-k^{2}}\left|\eta_{s}\right|_{\mathbf{R}^{m}}, \quad\left\langle p^{1}-\eta_{s}, \nu\right\rangle \leq-\frac{1}{1+\|\mathcal{B}\|_{\infty}} \sqrt{1-k^{2}}\left|p^{1}-\eta_{s}\right|_{\mathbf{R}^{m}}
$$

Repeating this argument for each $s$, we obtain that there are $\eta_{1}, \ldots, \eta_{n} \in \mathbf{R}^{m}$ such that (33) holds. 
Hence the proof of lemma is complete.

Now we are able to show the proof of Theorem 4.1.

Proof of Theorem 4.1. Possibly modifying $E$ in a $\mathcal{L}^{m+n}$-negligible set, we can assume that $E$ coincides with the set of points where $E$ has positive lower density. Precisely

$$
E=\left\{p \in \mathbf{G}: \liminf _{\delta \rightarrow 0} \frac{\mathcal{L}^{m+n}\left(E \cap \mathcal{U}_{e}(p, \delta)\right)}{\mathcal{L}^{m+n}\left(\mathcal{U}_{e}(p, \delta)\right)}>0\right\}
$$

where $\mathcal{U}_{e}(p, \delta)$ is the Euclidean ball centered at $p$ having radius $\delta>0$.

Let $\beta=\beta(k)>0$ as in Lemma 4.4. We would like to show that for every $p \in E$

$$
\left\{q \in \mathcal{U}(0, r):\left\|\nu^{\perp}\left(p^{-1} q\right)\right\|<-\beta\left\langle p^{-1} q, \nu\right\rangle\right\} \subset E .
$$

First we define

$$
\mathcal{A}_{1}(p):=\left\{\exp t Z_{\mu}(p) \in \mathcal{U}(0, r): t \geq 0, \mu \in \mathbf{S}_{k}^{m-1}\right\}
$$

where $\mathbf{S}_{k}^{m-1}:=\left\{\mu \in \mathbf{S}^{m-1}:\langle\mu, \nu\rangle \leq-\frac{1}{1+\|\mathcal{B}\|_{\infty}} \sqrt{1-k^{2}}\right\},\|\mathcal{B}\|_{\infty}=\max \left\{b_{j l}^{(s)}: s=\right.$ $1, \ldots n$ and $j, l=1, \ldots, m\}$ and $Z_{\mu}$ is the left invariant vector field

$$
Z_{\mu}=\mu_{1} X_{1}+\cdots+\mu_{m} X_{m}, \quad \text { for } \mu=\left(\mu_{1}, \ldots, \mu_{m}\right) \in \mathbf{S}_{k}^{m-1} .
$$

For any $\xi \in \mathbf{C}_{c}^{1}(\mathcal{U}(0, r), \mathbf{R})$ such that $\xi \geq 0$ and for all $\mu \in \mathbf{S}_{k}^{m-1}$ it follows

$$
\int_{E} Z_{\mu} \xi d \mathcal{L}^{m+n}=-\int_{\mathcal{U}(0, r)} \xi\left\langle\mu, \nu_{E}\right\rangle d|\partial E|_{\mathbf{G}} \leq 0 .
$$

Indeed we know that $\left\langle\mu, \nu_{E}(p)\right\rangle \geq 0$ for $|\partial E|_{\mathbf{G}^{-}}$a.e. $p \in \mathcal{U}(0, r)$ because $\langle\mu, \nu\rangle \leq$ $-\frac{1}{1+\|\mathcal{B}\|_{\infty}} \sqrt{1-k^{2}}$ and $\left\langle\nu_{E}, \nu\right\rangle \leq-k$. Then using Lemma 4.3 we conclude that if $p \in E \cap \mathcal{U}(0, r), t>0$ is such that $\exp t Z_{\mu}(p) \in \mathcal{U}(0, r)$ and $\delta>0$ is small enough, then

$$
\mathcal{L}^{m+n}\left(E \cap \exp t Z_{\mu}\left(\mathcal{U}_{e}(p, \delta)\right)\right) \geq \mathcal{L}^{m+n}\left(E \cap \mathcal{U}_{e}(p, \delta)\right) .
$$

Moreover, by $\mathcal{L}^{m+n}\left(\exp t Z_{\mu}\left(\mathcal{U}_{e}(p, \delta)\right)\right)=\mathcal{L}^{m+n}\left(\mathcal{U}_{e}(p, \delta)\right)$ we deduce

$$
\liminf _{\delta \rightarrow 0} \frac{\mathcal{L}^{m+n}\left(E \cap \exp t Z_{\mu}\left(\mathcal{U}_{e}(p, \delta)\right)\right)}{\mathcal{L}^{m+n}\left(\exp t Z_{\mu}\left(\mathcal{U}_{e}(p, \delta)\right)\right)} \geq \liminf _{\delta \rightarrow 0} \frac{\mathcal{L}^{m+n}\left(E \cap \mathcal{U}_{e}(p, \delta)\right)}{\mathcal{L}^{m+n}\left(\mathcal{U}_{e}(p, \delta)\right)}>0
$$

and consequently the point $q=\exp t Z_{\mu}(p)$ satisfies

$$
\liminf _{\delta \rightarrow 0} \frac{\mathcal{L}^{m+n}\left(E \cap \mathcal{U}_{e}(q, \delta)\right)}{\mathcal{L}^{m+n}\left(\mathcal{U}_{e}(q, \delta)\right)}>0 .
$$

This implies that $\exp t Z_{\mu}(p) \in E$ and $\mathcal{A}_{1}(p) \subset E$. Now if $p=0 \in E$, then

$$
\begin{aligned}
\mathcal{A}_{1}(0) & =\left\{\exp t Z_{\mu}(0) \in \mathcal{U}(0, r): t \geq 0, \mu \in \mathbf{S}_{k}^{m-1}\right\} \\
& =\left\{(\eta, 0) \in \mathbf{G}: \eta \in \mathbf{R}^{m},\langle\eta, \nu\rangle \leq-\frac{1}{1+\|\mathcal{B}\|_{\infty}}|\eta| \sqrt{1-k^{2}},|\eta|<r\right\} .
\end{aligned}
$$

Moreover, if we consider the conditions (33) of Lemma 4.4 and we define

$$
\mathcal{A}_{2}:=\left\{\exp t Z_{\mu}(\eta, 0) \in \mathcal{U}(0, r): t \geq 0, \mu \in \mathbf{S}_{k}^{m-1},(\eta, 0) \in \mathcal{A}_{1}(0)\right\}
$$

then

$$
\mathcal{A}_{2}=\left\{\left(p^{1}, p^{2}\right) \in \mathbf{G}: \text { there are } \eta_{1}, \ldots, \eta_{n} \in \mathbf{R}^{m},\left|\eta_{s}\right|_{\mathbf{R}^{m}}<r \text { such that (33) holds }\right\}
$$

and $\mathcal{A}_{2} \subset E$. Hence by Proposition 4.4 we obtain

$$
\left\{q \in \mathcal{U}(0, r):\left\|\nu^{\perp}(q)\right\|<-\beta\langle q, \nu\rangle\right\} \subset \mathcal{A}_{2} \subset E,
$$


i.e. (27) is true in the case $p=0$. The inclusion (27) follows for each $p \in E$ from the case $p=0$ by a left translation.

Now we consider $\mathbf{G}-E$ where $\nu_{\mathrm{G}-E}=-\nu_{E}$ in $\mathcal{U}(0, r)$. We can repeat the previous argument and we obtain for each $P$ where $\mathbf{G}-E$ has positive lower density,

$$
\left\{q \in \mathcal{U}(0, r):\left\|\nu^{\perp}\left(p^{-1} q\right)\right\|<\beta\left\langle p^{-1} q, \nu\right\rangle\right\} \subset \mathbf{G}-E .
$$

Precisely (41) holds for all $p \in \mathcal{U}(0, r)-E$ because $\mathbf{G}-E$ has density 1 at such $p$.

Approximating a point $p \in \partial E \cap \mathcal{U}(0, r)$ with a sequence of points in $E \cap \mathcal{U}(0, r)$, from (40) we obtain (27). Moreover, approximating a point $p$ with a sequence of points in $\mathcal{U}(0, r)-E$, using (41), (28) holds. Possibly we have to take a smaller $\beta$.

Finally we are able to show the proof of Theorem 4.2.

Proof of Theorem 4.2. Let $\mathbf{P}_{\nu^{\perp}}: \mathbf{G} \rightarrow \nu^{\perp}$ be the projection map. By (40) we have that $\mathbf{P}_{\nu^{\perp}}(E \cap \mathcal{U}(0, r))$ is open set in $\mathbf{P}_{\nu^{\perp}}(\mathcal{U}(0, r))$ and relatively open in $\nu^{\perp}$. Let

$$
\hat{\mathcal{O}}:=\left\{p \in \mathbf{P}_{\nu^{\perp}}(E \cap \mathcal{U}(0, r)): \text { there is } t \in \mathbf{R} \text { such that } \exp t Z_{\nu}(p) \in \mathcal{U}(0, r)-E\right\} .
$$

By (40) and (41) we deduce that $\hat{\mathcal{O}}$ is relatively open in $\mathbf{P}_{\nu^{\perp}}(\mathcal{U}(0, r))$ and so in $\nu^{\perp}$. Then from Theorem 4.1, the function $\hat{\phi}=\phi \nu: \hat{\mathcal{O}} \rightarrow \mathbf{G}$ (see (14)) defined as

$$
\phi(p):=\sup \left\{t \in \mathbf{R}: \exp t Z_{\nu}(p) \in \mathcal{U}(0, r) \text { and } \chi_{E}\left(\exp t Z_{\nu}(p)\right)=1\right\},
$$

is intrinsic Lipschitz map because graph $(\hat{\phi}) \cap\left\{Q \in \mathcal{U}(0, r):\left\|\nu^{\perp}\left(p^{-1} q\right)\right\|<\beta\left\langle p^{-1} q, \nu\right\rangle\right\}=$ $\emptyset$ (see Definition 2.11) and

$$
\partial E \cap \mathcal{U}(0, r)=\{p \cdot \hat{\phi}(p): p \in \mathcal{O}\}
$$

i.e. the thesis is true.

\section{Area formula in Carnot groups of step 2}

Area formula with respect to the spherical Hausdorff measure for the graph of an intrinsic Lipschitz function has been obtained in the context of Heisenberg groups in [9]. Here we give the area formula with respect to the spherical Hausdorff measure for the intrinsic Lipschitz graph in Carnot groups of step 2 (see Theorem 5.7); the proof is based on Theorem 1.6 in [9]. Moreover, Proposition 5.6 proves that the pointwise gradient coincides with the weak one; this statement is the counterpart of Proposition 4.7 in [9] inside Heisenberg groups. We observe that this fact is not elementary at all in our situation, since the $\nabla^{\hat{\phi}} \hat{\phi}$ is not well defined when $\hat{\phi}$ is an intrinsic Lipschitz function; however, we emphasize the fact that it exists almost everywhere: indeed if $\hat{\phi}$ is an intrinsic Lipschitz function, then by Theorem 2.20 we know that $\hat{\phi}$ is intrinsic differentiable a.e. in $\hat{\mathcal{O}}$ and, for all point of intrinsic differentiability of $\hat{\phi}$, there exists a unique intrinsic differential of $\hat{\phi}$ at this point defined as (6).

In this section we examine a Carnot group $\mathbf{G}$ of step 2 and $\mathbf{V}, \mathbf{W}$ are the complementary subgroups defined in (13).

Let $\hat{\phi}: \hat{\mathcal{O}} \rightarrow \mathbf{V}$ be intrinsic Lipschitz function, where $\hat{\mathcal{O}}$ is an open in $\mathbf{W}$ and $\phi: \mathcal{O} \rightarrow \mathbf{R}$ is the map associated to $\hat{\phi}$ as in (14). We recall that $\Phi: \mathcal{O} \rightarrow \mathbf{G}$ is the graph map of $\hat{\phi}$ defined as

$$
\Phi(a):=i(a) \cdot \hat{\phi}(i(a))
$$


In [16], the authors show the following proposition about the intrinsic subgraph of an intrinsic Lipschitz map:

$$
\mathcal{E}=\mathcal{E}_{\hat{\phi}}:=\{i(a) \cdot(t, 0, \ldots, 0) \in \hat{\mathcal{O}} \cdot \mathbf{V}: t<\phi(a)\} .
$$

Proposition 5.1. [16, Theorem 4.2.9] If $\hat{\phi}: \hat{\mathcal{O}} \rightarrow \mathbf{V}$ is intrinsic Lipschitz, then the subgraph $\mathcal{E}$ of $\hat{\phi}$ is a set with locally finite $\mathbf{G}$-perimeter.

We begin with a result about the intrinsic generalized inward normal $\nu_{\mathcal{E}}$ (see Section 2.2) to the intrinsic subgraph:

Lemma 5.2. Let $\mathbf{G}:=\left(\mathbf{R}^{m+n}, \cdot, \delta_{\lambda}\right)$ be a Carnot group of step 2 and $\mathbf{V}, \mathbf{W}$ the complementary subgroups defined in (13). Let $\hat{\phi}: \hat{\mathcal{O}} \rightarrow \mathbf{V}$ be an intrinsic Lipschitz function, where $\hat{\mathcal{O}}$ is an open subset of $\mathbf{W}$ and $\phi: \mathcal{O} \rightarrow \mathbf{R}$ is the map associated to $\hat{\phi}$ as in (14). Then the intrinsic generalized inward normal $\nu_{\mathcal{E}}$ to the intrinsic subgraph $\mathcal{E}$ in $\mathbf{G}$ has the following representation

$$
\nu_{\mathcal{E}}(\Phi(a))=\left(-\frac{1}{\sqrt{1+\left|\nabla^{\hat{\phi}} \hat{\phi}(i(a))\right|_{\mathbf{R}^{m-1}}^{2}}}, \frac{\nabla^{\hat{\phi}} \hat{\phi}(i(a))}{\sqrt{1+\left|\nabla^{\hat{\phi}} \hat{\phi}(i(a))\right|_{\mathbf{R}^{m-1}}^{2}}}\right)
$$

for a.e. $a \in \mathcal{O}$.

Proof. By Theorem 2.20, Theorem 2.5 and Theorem 2.10 we have that $\hat{\phi}$ is intrinsic differentiable a.e. in $\hat{\mathcal{O}}$ and for all $i(a) \in \hat{\mathcal{O}}$ point of intrinsic differentiability of $\hat{\phi}$, there exists a unique $d \hat{\phi}_{i(a)}: \mathbf{W} \rightarrow \mathbf{V}$ intrinsic differential of $\hat{\phi}$ at $i(a)$ such that

$$
\operatorname{graph}\left(d \hat{\phi}_{i(a)}\right)=\left\{\left(p_{1}, \ldots, p_{m+n}\right) \in \mathbf{G}: \sum_{j=1}^{m} \nu_{\mathcal{E}}^{(j)}(\Phi(a)) p_{j}=0\right\}
$$

where $\nu_{\mathcal{E}}^{(1)}, \ldots, \nu_{\mathcal{E}}^{(m)}$ are the components of $\nu_{\mathcal{E}}$. Then we obtain

$$
\left\{b \cdot d \hat{\phi}_{i(a)}(b) \in \mathbf{G}: b \in \mathbf{W}\right\}=\left\{\left(p_{1}, \ldots, p_{m+n}\right) \in \mathbf{G}: \sum_{j=1}^{m} \nu_{\mathcal{E}}^{(j)}(\Phi(a)) p_{j}=0\right\} .
$$

By (6) there is $\left(D_{2}^{\phi} \phi(a), \ldots, D_{m}^{\phi} \phi(a)\right) \in \mathbf{R}^{m-1}$ associated to $d \hat{\phi}_{i(a)}$ such that

$$
d \hat{\phi}_{i(a)}(b)=\left(\sum_{j=2}^{m} D_{j}^{\phi} \phi(a) x_{j}, 0, \ldots, 0\right)
$$

for all $b=\left(0, x_{2}, \ldots, x_{m}, y_{1}, \ldots, y_{n}\right) \in \mathbf{W}$, and consequently recalling that $(b$. $\left.d \hat{\phi}_{i(a)}(b)\right)^{1}=\left(\sum_{j=2}^{m} D_{j}^{\phi} \phi(a) x_{j}, x_{2}, \ldots, x_{m}\right)$ we deduce

$$
\nu_{\mathcal{E}}^{(1)}(\Phi(a)) \sum_{j=2}^{m} D_{j}^{\phi} \phi(a) x_{j}+\sum_{j=2}^{m} \nu_{\mathcal{E}}^{(j)}(\Phi(a)) x_{j}=0
$$

for all $b=\left(0, x_{2}, \ldots, x_{m}, y_{1}, \ldots, y_{n}\right) \in \mathbf{W}$. The thesis follows choosing $b=(0, \ldots, 0$, $1,0, \ldots, 0)$ where $j$-th element is 1 for $j=2, \ldots, m$ and recalling that $\left|\nu_{\mathcal{E}}(\Phi(a))\right|_{\mathbf{R}^{m}}=$ 1 a.e. in $\mathcal{O}$.

Using the fact that every intrinsic Lipschitz function is differentiable almost everywhere, we have the following proposition.

Proposition 5.3. Let $\mathbf{G}:=\left(\mathbf{R}^{m+n}, \cdot, \delta_{\lambda}\right)$ be a Carnot group of step 2 and $\mathbf{V}$, W the complementary subgroups defined in (13). Let $\hat{\phi}: \hat{\mathcal{O}} \rightarrow \mathbf{V}$ be an intrinsic $C_{L}$-Lipschitz function, where $\mathcal{O}$ is open and bounded in $\mathbf{W}$ and $\phi: \mathcal{O} \rightarrow \mathbf{R}$ is the 
map associated to $\hat{\phi}$ as in (14). Then the intrinsic gradient $\nabla^{\hat{\phi}} \hat{\phi}$, which is defined $\mathcal{L}^{m+n-1}$-a.e. in $\mathcal{O}$, satisfies

$$
\left\|\nabla^{\hat{\phi}} \hat{\phi}\right\|_{\mathcal{L}^{\infty}\left(\mathcal{O}, \mathbf{R}^{m-1}\right)} \leq C \quad \mathcal{L}^{m+n-1} \text {-a.e. } a \in \mathcal{O},
$$

where $C=C\left(n, m, C_{L}, \mathcal{B}^{(s)}\right)>0$. As a consequence $\nabla^{\hat{\phi}} \hat{\phi} \in \mathcal{L}^{\infty}\left(\mathcal{O}, \mathbf{R}^{m-1}\right)$.

Proof. Firstly we define, for all $j=2 \ldots, m$ and $a \in \mathcal{O}$, the exponential map of the vector field $D_{j}^{\phi}[-\delta, \delta] \ni t \mapsto \gamma_{a}^{j}(t):=\exp \left(t D_{j}^{\phi}\right)(a) \in \mathcal{O}$ as

$$
\left\{\begin{array}{l}
\dot{\gamma}_{a}^{j}=D_{j}^{\phi} \circ \gamma_{a}^{j} \\
\gamma_{a}^{j}(0)=a
\end{array}\right.
$$

This map is well-defined for $\delta>0$ small enough. Let us now fix $t \in[-\delta, \delta]$ and $j=2, \ldots, m$ and consider $h(s):=t e_{j}$ and $\gamma_{h}(s):=\exp \left(t s D_{j}^{\phi}\right)(a)$ if $s \in[0,1]$. Then by $(25)$ and $(26)$

$$
\left\|\hat{\phi}\left(i\left(\gamma_{a}^{j}(t)\right)\right)^{-1} i\left(\gamma_{a}^{j}(t)\right)^{-1} a \hat{\phi}\left(i\left(\gamma_{a}^{j}(t)\right)\right)\right\| \leq C|t|, \quad \forall t \in[-\delta, \delta],
$$

for every $j=2, \ldots, m$.

Finally using the same argument contained in the proof of Proposition 5.6 in [11] (see Proposition 3.7 in [3] in the context of Heisenberg groups), it can be proved that, at each point $a \in \mathcal{O}$ where $\phi$ is intrinsic differentiable

$$
\nabla_{j}^{\hat{\phi}} \hat{\phi}(a)=\lim _{t \rightarrow 0} \frac{\phi\left(\exp \left(t D_{j}^{\phi}\right)(a)\right)-\phi(a)}{t} \quad \forall j=2, \ldots, m .
$$

In the end by this equality and (45), (44) holds.

Lemma 5.4. [16, Lemma 4.2.10] Let $\hat{\phi}: \hat{\mathcal{O}} \rightarrow \mathbf{V}$ be an intrinsic Lipschitz function. Then there exists $C(\mathbf{W}, \mathbf{V})>0$ such that

$$
(\Phi)_{*}\left(\mathcal{L}^{m+n-1} \mathbf{L} \mathbf{W}\right)=-C(\mathbf{W}, \mathbf{V}) \nu_{\mathcal{E}}^{(1)}|\partial \mathcal{E}|_{\mathbf{G}},
$$

where $(\Phi)_{*}\left(\mathcal{L}^{m+n-1}\llcorner\mathbf{W})\right.$ denotes the image of $\mathcal{L}^{m+n-1}\llcorner\mathbf{W}$ under the map $\Phi$ and $\nu_{\mathcal{E}}^{(1)}$ is the first component of the intrinsic generalized inward normal to the intrinsic subgraph $\mathcal{E}$.

Proposition 5.5. Let $\mathbf{G}:=\left(\mathbf{R}^{m+n}, \cdot, \delta_{\lambda}\right)$ be a Carnot group of step 2 and $\mathbf{V}$, $\mathbf{W}$ the complementary subgroups defined in (13). Let $\hat{\phi}: \hat{\mathcal{O}} \rightarrow \mathbf{V}$ be an intrinsic Lipschitz function, where $\hat{\mathcal{O}}$ is an open subset of $\mathbf{W}$ and $\phi: \mathcal{O} \rightarrow \mathbf{R}$ is the map associated to $\hat{\phi}$ as in (14). Then for every $\xi=\left(\xi_{1}, \ldots, \xi_{m}\right) \in \mathbf{C}_{c}^{1}\left(\hat{\mathcal{O}} \cdot \mathbf{V}, \mathbf{R}^{m}\right)$ we have

$$
\int_{\mathcal{E}} \operatorname{div}_{\mathbf{G}} \xi d \mathcal{L}^{m+n}=\frac{1}{C(\mathbf{W}, \mathbf{V})} \int_{\mathcal{O}} \xi_{1} \circ \Phi-\sum_{j=2}^{m} D_{j}^{\phi} \phi(\xi \circ \Phi)_{j} d \mathcal{L}^{m+n-1},
$$

where $C(\mathbf{W}, \mathbf{V})>0$ is given by Lemma 5.4 and $\Phi: \mathcal{O} \rightarrow \mathbf{G}$ is the graph map of $\hat{\phi}$.

Proof. Thanks to Proposition 5.1 we know that $\mathcal{E}$ is a set of locally finite perimeter in $\mathbf{G}$ and consequently by Structure Theorem of $\mathrm{BV}_{\mathbf{G}}$ functions (see Theorem 2.1) there exists a unique $|\partial \mathcal{E}|_{\mathbf{G}}$-measurable function $\nu_{\mathcal{E}}: \hat{\mathcal{O}} \cdot \mathbf{V} \rightarrow \mathbf{R}^{m}$ such that $\left|\nu_{\mathcal{E}}\right|_{\mathbf{R}^{m}}=1$ $|\partial \mathcal{E}|_{\mathbf{G}}$-a.e. in $\hat{\mathcal{O}} \cdot \mathbf{V}$ and

$$
\int_{\mathcal{E}} \operatorname{div}_{\mathbf{G}} \xi d \mathcal{L}^{m+n}=-\int_{\hat{\mathcal{O}} \cdot \mathbf{V}}\left\langle\xi, \nu_{\mathcal{E}}\right\rangle d|\partial \mathcal{E}|_{\mathbf{G}}
$$


for all $\xi \in \mathbf{C}_{c}^{1}\left(\hat{\mathcal{O}} \cdot \mathbf{V}, \mathbf{R}^{m}\right)$ with $|\xi|_{\mathbf{R}^{m}} \leq 1$. Now using the Lemma 5.2 we have that the first component $\nu_{\mathcal{E}}^{(1)}$ of $\nu_{\mathcal{E}}$ is such that $\nu_{\mathcal{E}}^{(1)} \neq 0|\partial \mathcal{E}|_{\mathbf{G}^{-}}$-a.e. in $\hat{\mathcal{O}} \cdot \mathbf{V}$. Moreover, from Lemma 5.4 there exists $C(\mathbf{W}, \mathbf{V})>0$ such that

$$
\begin{aligned}
-\int_{\hat{\mathcal{O}} \cdot \mathbf{V}}\left\langle\xi, \nu_{\mathcal{E}}\right\rangle d|\partial \mathcal{E}|_{\mathbf{G}} & =-\int_{\hat{\mathcal{O}} \cdot \mathbf{V}} \frac{\left\langle\xi, \nu_{\mathcal{E}}\right\rangle}{\nu_{\mathcal{E}}^{(1)}} \nu_{\mathcal{E}}^{(1)} d|\partial \mathcal{E}|_{\mathbf{G}} \\
& =\frac{1}{C(\mathbf{W}, \mathbf{V})} \int_{\hat{\mathcal{O}} \cdot \mathbf{V}} \frac{\left\langle\xi, \nu_{\mathcal{E}}\right\rangle}{\nu_{\mathcal{E}}^{(1)}} d \Phi_{*}\left(\mathcal{L}^{m+n-1} \mathbf{L} \mathbf{W}\right) .
\end{aligned}
$$

Finally by a change of variables we conclude that

$$
\frac{1}{C(\mathbf{W}, \mathbf{V})} \int_{\hat{\mathcal{O}} \cdot \mathbf{V}} \frac{\left\langle\xi, \nu_{\mathcal{E}}\right\rangle}{\nu_{\mathcal{E}}^{(1)}} d \Phi_{*}\left(\mathcal{L}^{m+n-1} \mathbf{L}\right)=\frac{1}{C(\mathbf{W}, \mathbf{V})} \int_{\mathcal{O}} \frac{\left\langle\nu_{\mathcal{E}} \circ \Phi, \xi \circ \Phi\right\rangle}{\nu_{\mathcal{E}}^{(1)} \circ \Phi} d \mathcal{L}^{m+n-1}
$$

and consequently, by (43) for all $\xi=\left(\xi_{1}, \ldots, \xi_{m}\right) \in \mathbf{C}_{c}^{1}\left(\hat{\mathcal{O}} \cdot \mathbf{V}, \mathbf{R}^{m}\right)$ with $|\xi|_{\mathbf{R}^{m}} \leq 1$

$$
\begin{aligned}
\int_{\mathcal{E}} \operatorname{div}_{\mathbf{G}} \xi d \mathcal{L}^{m+n} & =-\int_{\hat{\mathcal{O}} \cdot \mathbf{V}}\left\langle\xi, \nu_{\mathcal{E}}\right\rangle d|\partial \mathcal{E}|_{\mathbf{G}} \\
& =\frac{1}{C(\mathbf{W}, \mathbf{V})} \int_{\mathcal{O}} \frac{\left\langle\nu_{\mathcal{E}} \circ \Phi, \xi \circ \Phi\right\rangle}{\nu_{\mathcal{E}}^{(1)} \circ \Phi} d \mathcal{L}^{m+n-1} \\
& =\frac{1}{C(\mathbf{W}, \mathbf{V})} \int_{\mathcal{O}}\left(\xi_{1} \circ \Phi+\sum_{j=2}^{m} \frac{\left(\nu_{\mathcal{E}} \circ \Phi\right)_{j}}{\nu_{\mathcal{E}}^{(1)} \circ \Phi}(\xi \circ \Phi)_{j}\right) d \mathcal{L}^{m+n-1} \\
& =\frac{1}{C(\mathbf{W}, \mathbf{V})} \int_{\mathcal{O}}\left(\xi_{1} \circ \Phi-\sum_{j=2}^{m} D_{j}^{\phi} \phi(\xi \circ \Phi)_{j}\right) d \mathcal{L}^{m+n-1}
\end{aligned}
$$

Hence putting together the last equality and (46) we obtain the thesis.

Now we are going to prove that the intrinsic gradient of an intrinsic Lipschitz function also agrees with the distributional gradient.

Proposition 5.6. Let $\mathbf{G}:=\left(\mathbf{R}^{m+n}, \cdot, \delta_{\lambda}\right)$ be a Carnot group of step 2 and $\mathbf{V}, \mathbf{W}$ the complementary subgroups defined in (13). Let $\hat{\phi}: \hat{\mathcal{O}} \rightarrow \mathbf{V}$ be an intrinsic Lipschitz function, where $\hat{\mathcal{O}}$ is an open subset of $\mathbf{W}$ and $\phi: \mathcal{O} \rightarrow \mathbf{R}$ is the map associated to $\hat{\phi}$ as in (14). Then the intrinsic gradient $\left(D_{2}^{\phi} \phi, \ldots, D_{m}^{\phi} \phi\right)$ is also distributional, i.e.

$$
\int_{\mathcal{O}} \phi\left(X_{j} \zeta+\phi \sum_{s=1}^{n} b_{j 1}^{(s)} Y_{s} \zeta\right) d \mathcal{L}^{m+n-1}=-\int_{\mathcal{O}} D_{j}^{\phi} \phi \zeta d \mathcal{L}^{m+n-1}
$$

for every $\zeta \in \mathbf{C}_{c}^{1}(\mathcal{O}, \mathbf{R})$ and $j=2, \ldots, m$.

Proof. Let $M:=\|\phi\|_{\mathcal{L}^{\infty}(\mathcal{O}, \mathbf{R})}<+\infty$. By standard considerations there is a sequence $\left(\phi_{h}\right)_{h \in \mathbf{N}} \subset \mathbf{C}_{c}^{\infty}(\mathcal{O}, \mathbf{R})$ converging uniformly to $\phi$ on each $\mathcal{O}^{\prime} \Subset \mathcal{O}$. We would like to prove that the sequence $\left(D_{2}^{\phi_{h}} \phi_{h}, \ldots, D_{m}^{\phi_{h}} \phi_{h}\right)_{h \in \mathbf{N}}$ converges to $\left(D_{2}^{\phi} \phi, \ldots, D_{m}^{\phi} \phi\right)$ in the sense of distributions on each $\mathcal{O}^{\prime} \Subset \mathcal{O}$. Indeed, we start to show that

$$
\int_{\mathcal{O}} \sum_{j=2}^{m} D_{j}^{\phi} \phi \eta_{j} d \mathcal{L}^{m+n-1}=\lim _{h \rightarrow \infty} \int_{\mathcal{O}} \sum_{j=2}^{m} D_{j}^{\phi_{h}} \phi_{h} \eta_{j} d \mathcal{L}^{m+n-1} \quad \forall \eta \in \mathbf{C}_{c}^{1}\left(\mathcal{O}, \mathbf{R}^{m-1}\right) .
$$

We denote by $\Phi_{h}: \mathcal{O} \rightarrow \mathbf{G}$ the graph map of $\hat{\phi}_{h}=\left(\phi_{h}, 0, \ldots, 0\right)$ defined as $\Phi_{h}(a):=$ $i(a) \hat{\phi}_{h}(i(a))$ and $\mathcal{E}_{h}$ the intrinsic subgraph of $\hat{\phi}_{h}$. Therefore, by Proposition 5.5 we 
know that for each $\xi=\left(\xi_{1}, \ldots, \xi_{m}\right) \in \mathbf{C}_{c}^{1}\left(\hat{\mathcal{O}} \cdot \mathbf{V}, \mathbf{R}^{m}\right)$

$$
\int_{\mathcal{E}} \operatorname{div}_{\mathbf{G}} \xi d \mathcal{L}^{m+n}=\frac{1}{C(\mathbf{W}, \mathbf{V})} \int_{\mathcal{O}} \xi_{1} \circ \Phi-\sum_{j=2}^{m} D_{j}^{\phi} \phi(\xi \circ \Phi)_{j} d \mathcal{L}^{m+n-1},
$$

where $C(\mathbf{W}, \mathbf{V})>0$ is given by Lemma 5.4. Moreover, by the uniform convergence of $\phi_{h}$ to $\phi$ we conclude that

$$
\int_{\mathcal{E}} \operatorname{div}_{\mathbf{G}} \xi d \mathcal{L}^{m+n}=\lim _{h \rightarrow \infty} \int_{\mathcal{E}_{h}} \operatorname{div}_{\mathbf{G}} \xi d \mathcal{L}^{m+n}
$$

Now we recall that $\mathbf{C}^{1}$ functions are uniformly intrinsic differentiable maps (see Theorem 2.12) and consequently they are also locally intrinsic Lipschitz maps (see Proposition 2.19). Hence we can apply Proposition 5.5 for every $\phi_{h}$ and we obtain that

$$
\int_{\mathcal{E}_{h}} \operatorname{div}_{\mathbf{G}} \xi d \mathcal{L}^{m+n}=\frac{1}{C(\mathbf{W}, \mathbf{V})} \int_{\mathcal{O}} \xi_{1} \circ \Phi_{h}-\sum_{j=2}^{m} D_{j}^{\phi_{h}} \phi_{h}\left(\xi \circ \Phi_{h}\right)_{j} d \mathcal{L}^{m+n-1}
$$

for every $\xi \in \mathbf{C}_{c}^{1}\left(\hat{\mathcal{O}} \cdot \mathbf{V}, \mathbf{R}^{m}\right)$. Finally, putting together the last three equalities we have that

$$
\begin{aligned}
& \frac{1}{C(\mathbf{W}, \mathbf{V})} \int_{\mathcal{O}} \xi_{1} \circ \Phi-\sum_{j=2}^{m} D_{j}^{\phi} \phi(\xi \circ \Phi)_{j} d \mathcal{L}^{m+n-1} \\
& =\lim _{h \rightarrow \infty} \frac{1}{C(\mathbf{W}, \mathbf{V})} \int_{\mathcal{O}} \xi_{1} \circ \Phi_{h}-\sum_{j=2}^{m} D_{j}^{\phi_{h}} \phi_{h}\left(\xi \circ \Phi_{h}\right)_{j} d \mathcal{L}^{m+n-1}
\end{aligned}
$$

for all $\xi \in \mathbf{C}_{c}^{1}\left(\hat{\mathcal{O}} \cdot \mathbf{V}, \mathbf{R}^{m}\right)$.

Clearly, if we choose $\xi_{1}=0$, then

$$
\int_{\mathcal{O}} \sum_{j=2}^{m} D_{j}^{\phi} \phi(\xi \circ \Phi)_{j} d \mathcal{L}^{m+n-1}=\lim _{h \rightarrow \infty} \int_{\mathcal{O}} \sum_{j=2}^{m} D_{j}^{\phi_{h}} \phi_{h}\left(\xi \circ \Phi_{h}\right)_{j} d \mathcal{L}^{m+n-1} .
$$

If we consider

$$
\xi_{j}(i(a) \cdot(t, 0, \ldots, 0)):=\eta_{j}(a) \rho(t)
$$

with $\eta=\left(\eta_{2}, \ldots, \eta_{m}\right) \in \mathbf{C}_{c}^{1}\left(\mathcal{O}, \mathbf{R}^{m-1}\right)$ and $\rho \in \mathbf{C}_{c}^{1}(\mathbf{R})$ such that $\rho(t)=1$ for all $t \in[-M-1, M+1]$, then $\xi=\left(0, \xi_{2}, \ldots, \xi_{m}\right) \in \mathbf{C}_{c}^{1}\left(\hat{\mathcal{O}} \cdot \mathbf{V}, \mathbf{R}^{m}\right)$ and by (49) we deduce that

$$
\begin{aligned}
& \int_{\mathcal{O}} \sum_{j=2}^{m} D_{j}^{\phi} \phi(a) \eta_{j}(a) \rho(\phi(a)) d \mathcal{L}^{m+n-1}(a) \\
& =\lim _{h \rightarrow \infty} \int_{\mathcal{O}} \sum_{j=2}^{m} D_{j}^{\phi_{h}} \phi_{h}(a) \eta_{j}(a) \rho\left(\phi_{h}(a)\right) d \mathcal{L}^{m+n-1}(a) .
\end{aligned}
$$

Hence since $\phi_{h}$ converges uniformly to $\phi$, there is $\bar{h} \in \mathbf{N}$ such that for all $h \geq \bar{h}$ and for all $a \in \operatorname{spt}(\eta)$ we have
(1) $\phi_{h}(a) \in[-M-1, M+1]$;
(2) $\rho\left(\phi_{h}(a)\right)=1$;

and consequently (48) follows. 
Now using again the uniformly convergence of $\phi_{h}$ to $\phi$ and (48) we conclude that for all $\eta=\left(\eta_{2}, \ldots, \eta_{m}\right) \in \mathbf{C}_{c}^{1}\left(\mathcal{O}, \mathbf{R}^{m-1}\right)$ and for all $j=2, \ldots, m$

$$
\begin{aligned}
\int_{\mathcal{O}} D_{j}^{\phi} \phi \eta_{j} d \mathcal{L}^{m+n-1} & =\lim _{h \rightarrow \infty} \int_{\mathcal{O}} D_{j}^{\phi_{h}} \phi_{h} \eta_{j} d \mathcal{L}^{m+n-1} \\
& =-\lim _{h \rightarrow \infty} \int_{\mathcal{O}} \phi_{h}\left(X_{j} \eta_{j}+\phi_{h} \sum_{s=1}^{n} b_{j 1}^{(s)} Y_{s} \eta_{j}\right) d \mathcal{L}^{m+n-1} \\
& =-\int_{\mathcal{O}} \phi\left(X_{j} \eta_{j}+\phi \sum_{s=1}^{n} b_{j 1}^{(s)} Y_{s} \eta_{j}\right) d \mathcal{L}^{m+n-1}
\end{aligned}
$$

Then the thesis follows with $\zeta=\eta_{j} \in \mathbf{C}_{c}^{1}(\mathcal{O}, \mathbf{R})$ for each $j=2, \ldots, m$.

The following result is only shown for specific choice of metric: the metric $d_{\infty}$.

Theorem 5.7. Let $\mathbf{G}:=\left(\mathbf{R}^{m+n}, \cdot, \delta_{\lambda}\right)$ be a Carnot group of step 2 and $\mathbf{V}, \mathbf{W}$ the complementary subgroups defined in (13). Let $\hat{\phi}: \hat{\mathcal{O}} \rightarrow \mathbf{V}$ be an intrinsic Lipschitz function, where $\hat{\mathcal{O}} \subset \mathbf{W}$ is an open set and $\phi: \mathcal{O} \rightarrow \mathbf{R}$ is the map associated to $\hat{\phi}$ as in (14). Then there exists $C(\mathbf{W}, \mathbf{V})>0$ such that the following area formula holds

$$
|\partial \mathcal{E}|_{\mathbf{G}}(\hat{\mathcal{O}} \cdot \mathbf{V})=\frac{1}{C(\mathbf{W}, \mathbf{V})} \int_{\mathcal{O}} \sqrt{1+|\nabla \hat{\phi} \hat{\phi}|_{\mathbf{R}^{m-1}}^{2}} d \mathcal{L}^{m+n-1} .
$$

Moreover, if $\mathbf{G}$ is endowed with a distance $d_{\infty}$, there exists $c=c(\mathbf{G})>0$ such that

$$
|\partial \mathcal{E}|_{\mathbf{G}}(\hat{\mathcal{O}} \cdot \mathbf{V})=c \mathcal{S}^{\mathfrak{q}-1}(\Phi(\mathcal{O}))
$$

where $\mathcal{S}^{\mathfrak{q}-1}$ is the spherical Hausdorff measure on $\mathbf{G}$ and $\mathfrak{q}=m+2 n$.

Proof. Thanks to Proposition 5.1 and Theorem 2.1 we know that $|\partial \mathcal{E}|_{\mathrm{G}}$ is a Radon measure. Then a classical approximation result ensures the existence of sequence $\left(\xi_{h}\right)_{h \in \mathbf{N}}=\left(\xi_{h, 1}, \ldots, \xi_{h, m}\right)_{h \in \mathbf{N}} \subset \mathbf{C}_{c}^{1}\left(\hat{\mathcal{O}} \cdot \mathbf{V}, \mathbf{R}^{m}\right)$ with $\left|\xi_{h}\right|_{\mathbf{R}^{m}} \leq 1$ such that

$$
\xi_{h} \rightarrow \nu_{\mathcal{E}} \quad|\partial \mathcal{E}|_{\mathbf{G}} \text {-a.e. in } \hat{\mathcal{O}} \cdot \mathbf{V}
$$

and by Lemma 5.2 and Lemma 5.4 we have that $\xi_{h} \circ \Phi \rightarrow \nu_{\mathcal{E}} \circ \Phi$ a.e. in $\mathcal{O}$.

Moreover, using (47) in Proposition 5.5 we get that for all $h \in \mathbf{N}$

$$
-\int_{\hat{\mathcal{O}} \cdot \mathbf{V}}\left\langle\xi_{h}, \nu_{\mathcal{E}}\right\rangle d|\partial \mathcal{E}|_{\mathbf{G}}=\frac{1}{C(\mathbf{W}, \mathbf{V})} \int_{\mathcal{O}} \xi_{h, 1} \circ \Phi-\sum_{j=2}^{m} D_{j}^{\phi} \phi\left(\xi_{h} \circ \Phi\right)_{j} d \mathcal{L}^{m+n-1} .
$$

Hence taking the limit as $h \rightarrow \infty$ in the last equality and by (50) and Lemma 5.2 we conclude

$$
|\partial \mathcal{E}|_{\mathbf{G}}(\hat{\mathcal{O}} \cdot \mathbf{V})=\frac{1}{C(\mathbf{W}, \mathbf{V})} \int_{\mathcal{O}} \sqrt{1+|\nabla \hat{\phi} \hat{\phi}|_{\mathbf{R}^{m-1}}^{2}} d \mathcal{L}^{m+n-1} .
$$

Finally, $|\partial \mathcal{E}|_{\mathbf{G}}(\hat{\mathcal{O}} \cdot \mathbf{V})=c \mathcal{S}^{\mathfrak{q}-1}(\Phi(\mathcal{O}))$ is a direct consequence of results of Theorem 2.4 and Theorem 2.5 (see also Theorem 1.3 in [31]).

\section{Smooth approximation for intrinsic lipschitz maps}

In this section we characterize intrinsic Lipschitz functions as maps which can be approximated by a sequence of smooth maps, with pointwise convergent intrinsic gradient. The classical approximation by convolution does not apply because of the nonlinearity of $\nabla^{\hat{\phi}} \hat{\phi}$. Here we use a similar technique in [9] in the context of Heisenberg groups. The strategy is somehow indirect, indeed we approximate in Carnot groups of step 2 the intrinsic subgraph of intrinsic Lipschitz function $\hat{\phi}$, rather 
than the function itself. The key point is that the intrinsic subgraph of $\hat{\phi}$ is a set of locally finite G-perimeter (see Proposition 5.1). Indeed, in a similar way as in Theorem 6.1 in [9], firstly we use Friedrichs' mollifier to regularize the characteristic function of the intrinsic subgraph of $\hat{\phi}$ with a family of functions $\left(f_{\alpha}\right)_{\alpha>0}$ which satisfies Theorem 2.13. Then we consider the level set of $\left(f_{\alpha}\right)_{\alpha>0}$ which defines a family of functions $\left(\phi_{\alpha}\right)_{\alpha>0}$. Finally we prove that $\left(\phi_{\alpha}\right)_{\alpha>0}$ is the family of functions, up to subsequence, that we were looking for.

Precisely, we prove the following theorem

Theorem 6.1. Let $\mathbf{G}:=\left(\mathbf{R}^{m+n}, \cdot, \delta_{\lambda}\right)$ be a Carnot group of step 2 and $\mathbf{V}$, $\mathbf{W}$ the complementary subgroups defined in (13). Let $\hat{\phi}: \hat{\mathcal{O}} \rightarrow \mathbf{V}$ be an intrinsic Lipschitz function, where $\hat{\mathcal{O}}$ is a bounded open subset of $\mathbf{W}$ and $\phi: \mathcal{O} \rightarrow \mathbf{R}$ is the map associated to $\hat{\phi}$ as in (14). Then there exists a sequence $\left(\hat{\phi}_{h}\right)_{h} \subset \mathbf{C}^{\infty}(\hat{\mathcal{O}}, \mathbf{V})$ such that

(1) $\hat{\phi}_{h}$ uniformly converges to $\hat{\phi}$ in $\hat{\mathcal{O}}$,

(2) $\left|\nabla^{\hat{\phi}_{h}} \hat{\phi}_{h}(a)\right| \leq\left\|\nabla^{\hat{\phi}} \hat{\phi}\right\|_{\mathcal{L}^{\infty}\left(\hat{\mathcal{O}}, \mathbf{R}^{m-1}\right)} \forall a \in \hat{\mathcal{O}}, h \in \mathbf{N}$,

(3) $\nabla^{\hat{\phi}_{h}} \hat{\phi}_{h}(a) \rightarrow \nabla^{\hat{\phi}} \hat{\phi}(a)$ a.e. $a \in \hat{\mathcal{O}}$.

Before stating the approximation theorem we need to recall the following results.

Theorem 6.2. [17, Theorem 4.0.5] Let $\mathbf{W}, \mathbf{V}$ be complementary subgroups of $\mathbf{G}$ with $\mathbf{V}$ horizontal and one dimensional. Let $\hat{\mathcal{O}}$ be a Borel subset of $\mathbf{W}$ and $\phi: \hat{\mathcal{O}} \rightarrow \mathbf{V}$ be an intrinsic $C_{L}$-Lipschitz function. Then there are $\psi: \mathbf{W} \rightarrow \mathbf{V}$ and $C=C\left(C_{L}, \mathbf{G}, \mathbf{W}, \mathbf{V}\right) \geq C_{L}$ such that

(1) $\psi$ is intrinsic $C$-Lipschitz in $\mathbf{W}$,

(2) $\psi(a)=\phi(a)$ for all $a \in \hat{\mathcal{O}}$.

Proposition 6.3. [17, Proposition 4.0.8] Let $\phi_{\beta}: \mathbf{W} \rightarrow \mathbf{V}$ be a family of intrinsic $C_{L}$-Lipschitz functions. There is $C=C\left(C_{L}, \mathbf{G}, \mathbf{W}, \mathbf{V}\right) \geq C_{L}>0$ such that if we put

$$
\phi:=\inf _{\beta \in I} \phi_{\beta}
$$

then either $\phi=-\infty$ or $\phi$ is defined on all of $\mathbf{W}$ and it is intrinsic C-Lipschitz. Analogously we can obtain the similar results for $\sup _{\beta \in I} \phi_{\beta}$.

Theorem 6.4. [39] Let $f: \mathbf{R}^{m+n} \rightarrow \mathbf{R}$ be a strictly convex function and let $\left(g_{h}\right)_{h}$ and $g$ be in $\mathcal{L}^{1}\left(\Omega, \mathbf{R}^{m+n}\right)$, where $\Omega \subset \mathbf{R}^{m+n}$. If

(1) $g_{h} \rightarrow g$ weakly in $\mathcal{L}^{1}\left(\Omega, \mathbf{R}^{m+n}\right)$,

(2) $\int_{\Omega} f \circ g_{h} d \mathcal{L}^{m+n} \rightarrow \int_{\Omega} f \circ g d \mathcal{L}^{m+n}$,

then $g_{h} \rightarrow g$ strongly in $\mathcal{L}^{1}\left(\Omega, \mathbf{R}^{m+n}\right)$.

Proof of Theorem 6.1. Without loss of generality, we can assume that $\hat{\phi}: \mathbf{W} \rightarrow$ V. Indeed, by Proposition $2.18(2), \hat{\phi}$ is locally uniformly continuous on $\hat{\mathcal{O}}$ and so we can extended it to a continuous function $\hat{\phi}: \operatorname{clos}(\hat{\mathcal{O}}) \rightarrow \mathbf{V}$. By Theorem 6.2 there exists a Lipschitz extension $\psi: \mathbf{W} \rightarrow \mathbf{V}$ of $\hat{\phi}$. Moreover, if we put $M:=\|\phi\|_{\mathcal{L}^{\infty}(\mathcal{O}, \mathbf{R})}<$ $+\infty$, then thanks to Proposition 6.3 the map $\psi^{*}: \mathbf{W} \rightarrow \mathbf{V}$ defined as

$$
\psi^{*}(a):=\max \{\min \{\psi(a),(M, 0, \ldots, 0)\},(-M, 0, \ldots, 0)\} \quad \text { for all } a \in \mathbf{W}
$$

is a bounded Lipschitz function, which still extends $\hat{\phi}$. Applying the following steps of the proof to $\psi^{*}$ we get the thesis.

We split the proof in several steps. 
Step 1. Let $\mathcal{E}:=\mathcal{E}_{\hat{\phi}}$ be the intrinsic subgraph of $\hat{\phi}: \mathbf{W} \rightarrow \mathbf{V}$ defined as (42). For every $\alpha>0$ we consider $f_{\alpha}: \mathbf{G} \rightarrow \mathbf{R}$ given by

$$
\begin{aligned}
f_{\alpha}(p) & :=\left(\rho_{\alpha} * \chi_{\mathcal{E}}\right)(p)=\int_{\mathbf{G}} \rho_{\alpha}\left(p q^{-1}\right) \chi_{\mathcal{E}}(q) d \mathcal{L}^{m+n}(q) \\
& =\int_{\mathbf{G}} \rho_{\alpha}(q) \chi_{\mathcal{E}}\left(q^{-1} p\right) d \mathcal{L}^{m+n}(q),
\end{aligned}
$$

where $\rho_{\alpha}(p):=\alpha^{m+2 n} \rho\left(\delta_{1 / \alpha}(p)\right)$ and $\rho \in \mathbf{C}_{c}^{\infty}(\mathcal{U}(0,1), \mathbf{R})$ is a smooth mollifier with $\rho\left(p^{-1}\right)=\rho(-p)=\rho(p)$ for every $p \in \mathbf{G}$.

By properties of convolution in $\mathbf{G}$ introduced in [15], we know that $f_{\alpha} \in \mathbf{C}_{c}^{\infty}(\mathbf{G}, \mathbf{R})$ and $\operatorname{spt}\left(f_{\alpha}\right) \subset \mathcal{U}(0, \alpha) \cdot \operatorname{spt}\left(\chi_{\mathcal{E}}\right)$ for every $\alpha>0$. Precisely, for all $\alpha>0$

$$
f_{\alpha}(p) \in[0,1] \text { for all } p \in \mathbf{G}
$$

and for all sufficiently small $\alpha>0$

$$
f_{\alpha}(p)=1 \quad \text { for all } p=\left(p_{1}, \ldots, p_{m+n}\right) \in \mathbf{G} \text { such that } p_{1} \leq-2 M
$$

Moreover, by definition of $\mathcal{E}$ we know that $\mathcal{E}$ is an open set in $\mathbf{G}$ and

$$
\operatorname{spt}\left(\chi_{\mathcal{E}}\right)=\operatorname{clos}(\mathcal{E}) \subset\{a \cdot(t, 0, \ldots, 0) \in \operatorname{clos}(\hat{\mathcal{O}}) \cdot \mathbf{V}: t \leq M\} .
$$

As a consequence

$$
\operatorname{spt}\left(f_{\alpha}\right) \subset \overline{\mathcal{U}(0, \alpha)} \cdot \operatorname{spt}\left(\chi_{\mathcal{E}}\right) \subset\{a \cdot(t, 0, \ldots, 0) \in \hat{\mathcal{O}} \cdot \mathbf{V}: t<2 M\} \quad \text { for } \alpha<M
$$

and

$$
f_{\alpha}(p)=0 \quad \text { for all } p=\left(p_{1}, \ldots, p_{m+n}\right) \in \mathbf{G} \text { such that } p_{1} \geq 2 M,
$$

i.e. $f_{\alpha}$ is constant far from the graph of $\hat{\phi}$, so (51) must be considered only in a neighborhood of the graphs itself.

Step 2. For every $\alpha>0$ and $c \in(0,1)$, let

$$
S_{\alpha}:=\left\{p=\left(p_{1}, \ldots, p_{m+n}\right) \in \hat{\mathcal{O}} \cdot \mathbf{V}: p_{1} \in(-2 M, 2 M), f_{\alpha}(p)=c\right\} .
$$

We note that if

$$
\operatorname{rank} \nabla_{\mathbf{G}} f_{\alpha}(p)=1, \quad \text { for all } p \in S_{\alpha}
$$

then $S_{\alpha}$ is the level set of a map $f_{\alpha} \in \mathbf{C}^{\infty}(\mathbf{G}, \mathbf{R})$ and so $f_{\alpha} \in \mathbf{C}_{\mathbf{G}}^{1}(\mathbf{G}, \mathbf{R})$ such that $\operatorname{rank} \nabla_{\mathbf{G}} f_{\alpha}$ is maximum, i.e. $S_{\alpha}$ is $\mathbf{G}$-regular hypersurface. More precisely, we show that

$$
X_{1} f_{\alpha}(p)<0 \text { for all } p \in S_{\alpha},
$$

where $X_{1} f_{\alpha}(p)$ is the first component of horizontal gradient $\nabla_{\mathbf{G}} f_{\alpha}$ of $f_{\alpha}$ in $p$. Indeed let $\mathcal{A}^{\prime}:=\left\{p=\left(p_{1}, \ldots, p_{m+n}\right) \in \hat{\mathcal{O}} \cdot \mathbf{V}: p_{1} \in(-3 M, 3 M)\right\}$ and $\varphi \in \mathbf{C}_{c}^{\infty}\left(\mathcal{A}^{\prime}, \mathbf{R}\right)$, then

$$
\begin{aligned}
\left\langle X_{1} f_{\alpha}, \varphi\right\rangle & =-\int_{\mathcal{A}^{\prime}} f_{\alpha}(q) X_{1} \varphi(q) d \mathcal{L}^{m+n}(q) \\
& =-\int_{\overline{\mathcal{U}(0, \alpha)}} \rho_{\alpha}(p) d \mathcal{L}^{m+n}(p) \int_{\mathcal{A}^{\prime}} \chi_{\mathcal{E}}\left(p^{-1} q\right) X_{1} \varphi(q) d \mathcal{L}^{m+n}(q) \\
& =-\int_{\overline{\mathcal{U}(0, \alpha)}} \rho_{\alpha}(p) d \mathcal{L}^{m+n}(p) \int_{\tau_{p^{-1}}\left(\mathcal{A}^{\prime}\right)} \chi_{\mathcal{E}}(q) X_{1} \varphi(p q) d \mathcal{L}^{m+n}(q)
\end{aligned}
$$


With the notation $\varphi_{p}(q):=\varphi(p q)$ we have $X_{1}(\varphi(p q))=X_{1} \varphi_{p}(q)$ because $X_{1}$ is left-invariant; moreover $\varphi_{p} \in \mathbf{C}_{c}^{\infty}\left(\tau_{p^{-1}} \mathcal{A}^{\prime}, \mathbf{R}\right)$ and by integration by parts, we obtain

$$
\int_{\tau_{p^{-1}}\left(\mathcal{A}^{\prime}\right)} \chi_{\mathcal{E}}(q) X_{1} \varphi_{p}(q) d \mathcal{L}^{m+n}(q)=-\int_{\tau_{p^{-1}}\left(\mathcal{A}^{\prime}\right)} \nu_{\mathcal{E}}^{(1)}(q) \varphi_{p}(q) d|\partial \mathcal{E}|_{\mathbf{G}}(q) .
$$

Since $\operatorname{spt}\left(\varphi_{p}\right) \Subset \tau_{p^{-1}}\left(\mathcal{A}^{\prime}\right)$ and $p \in \overline{\mathcal{U}(0, \alpha)}$ if $\alpha$ is small enough, we can replace $\tau_{p^{-1}}\left(\mathcal{A}^{\prime}\right)$ by $\mathcal{A}^{\prime}$. Thus by Fubini-Tonelli Theorem and a change of variable, we get

$$
\left\langle X_{1} f_{\alpha}, \varphi\right\rangle=\int_{\mathcal{A}^{\prime}} \nu_{\mathcal{E}}^{(1)}(q) d|\partial \mathcal{E}|_{\mathbf{G}}(q)\left(\int_{\mathbf{G}} \rho_{\alpha}(p) \varphi(p q) d \mathcal{L}^{m+n}(p)\right) .
$$

Then for all $p \in \mathcal{A}^{\prime}$ and for all $\alpha>0$ small enough

$$
\begin{aligned}
X_{1} f_{\alpha}(p) & =\int_{\mathcal{A}^{\prime}} \rho_{\alpha}\left(p q^{-1}\right) \nu_{\mathcal{E}}^{(1)}(q) d|\partial \mathcal{E}|_{\mathbf{G}}(q) \\
& =\int_{\mathcal{U}(0, \alpha) \cdot p} \rho_{\alpha}\left(p q^{-1}\right) \nu_{\mathcal{E}}^{(1)}(q) d|\partial \mathcal{E}|_{\mathbf{G}}(q) .
\end{aligned}
$$

In particular, we immediately deduce from (55) the following assertion: For each couple $\left(\hat{\mathcal{O}}, \hat{\mathcal{O}}_{0}\right)$ of open and bounded subsets of $\mathbf{W}$ with $\hat{\mathcal{O}} \Subset \hat{\mathcal{O}}_{0}$ there is $\bar{\alpha}=$ $\alpha\left(\hat{\mathcal{O}}_{0}\right)>0$ such that for every $0<\alpha<\bar{\alpha}$

$$
\int_{\mathcal{A}}\left|\nabla_{\mathbf{G}} f_{\alpha}\right| d \mathcal{L}^{m+n} \leq|\partial \mathcal{E}|_{\mathbf{G}}\left(\mathcal{A}_{0}\right)
$$

where $\mathcal{A}:=\left\{p=\left(p_{1}, \ldots, p_{m+n}\right) \in \hat{\mathcal{O}} \cdot \mathbf{V}: p_{1} \in(-2 M, 2 M)\right\}$ and $\mathcal{A}_{0}:=\{p=$ $\left.\left(p_{1}, \ldots, p_{m+n}\right) \in \hat{\mathcal{O}}_{0} \cdot \mathbf{V}: p_{1} \in(-2 M, 2 M)\right\}$. Moreover, since Lemma 5.2 holds, we get

and if we put

$$
\nu_{\mathcal{E}}^{(1)} \circ \Phi=-\frac{1}{\sqrt{1+\left|\nabla^{\hat{\phi}} \hat{\phi}\right|_{\mathbf{R}^{m-1}}^{2}}} \text { in } \mathcal{O}
$$

$$
I_{\alpha}(p):=\int_{\mathcal{U}(0, \alpha) \cdot p} \rho_{\alpha}\left(p q^{-1}\right) d|\partial \mathcal{E}|_{\mathbf{G}}(q),
$$

then using (55) we have

$$
X_{1} f_{\alpha}(p) \leq-\frac{1}{\sqrt{1+\left\|\nabla^{\hat{\phi}} \hat{\phi}\right\|_{\mathcal{L}^{\infty}\left(\hat{\mathcal{O}}, \mathbf{R}^{m-1}\right)}^{2}}} I_{\alpha}(p) \quad \text { for all } p \in \mathcal{A}^{\prime} .
$$

Moreover, for every fixed $c \in(0,1)$ and for all $p \in \mathcal{A}$ such that $f_{\alpha}(p)=c$, then

$$
\mathcal{L}^{m+n}((\mathcal{U}(0, \alpha) \cdot p) \cap \mathcal{E})>0 \quad \text { and } \quad \mathcal{L}^{m+n}\left((\mathcal{U}(0, \alpha) \cdot p) \cap \mathcal{E}^{c}\right)>0 .
$$

Indeed, by contradiction, if we assume $\mathcal{L}^{m+n}((\mathcal{U}(0, \alpha) \cdot p) \cap \mathcal{E})=0$. Then because $\mathcal{E}$ is open, we can assume $(\mathcal{U}(0, \alpha) \cdot p) \cap \mathcal{E}=\emptyset$. Hence by the definition of convolution, we have that $f_{\alpha}(p)=0 \neq c$ and then a contradiction. In a similar way it follows that $f_{\alpha}(p)=1$ if we suppose by contradiction $\mathcal{L}^{m+n}\left((\mathcal{U}(0, \alpha) \cdot p) \cap \mathcal{E}^{c}\right)=0$.

Now by (57) and by Theorem 2.2, we have that $|\partial \mathcal{E}|_{\mathbf{G}}((\mathcal{U}(0, \alpha) \cdot p)>0$ and $I_{\alpha}(p)>0$ for $p \in \mathcal{A}$ with $f_{\alpha}(p)=c$. As a consequence using (56), (54) holds and $S_{\alpha}$ is a G-regular hypersurface.

Step 3. Now we show that $\left(S_{\alpha}\right)_{\alpha}$ implicitly defines a sequence $\left(\hat{\phi}_{\alpha}\right)_{\alpha}$ with $\hat{\phi}_{\alpha}=$ $\left(\phi_{\alpha}, 0, \ldots, 0\right)$ and, up to subsequence, it is the family of functions which we were looking for. 
Fix $\alpha$ and $c \in(0,1)$. Because $f_{\alpha}$ is a continuous map such that

$$
\begin{aligned}
f_{\alpha}(i(a) \cdot(-2 M, 0, \ldots, 0)) & =1>c, \\
f_{\alpha}(i(a) \cdot(2 M, 0, \ldots, 0)) & =0<c
\end{aligned}
$$

for all $a \in \mathcal{O}$, there is $t_{a} \in(-2 M, 2 M)$ such that $f_{\alpha}\left(i(a) \cdot\left(t_{a}, 0, \ldots, 0\right)\right)=c$. In particular, since

$$
X_{1} f_{\alpha}(i(a) \cdot(t, 0, \ldots, 0)) \leq 0 \quad \forall t<t_{a} \quad \text { and } \quad X_{1} f_{\alpha}\left(i(a) \cdot\left(t_{a}, 0, \ldots, 0\right)\right)<0
$$

we have that $t_{a}$ is unique and $\left\{t \in(-2 M, 2 M): f_{\alpha}(i(a) \cdot(t, 0, \ldots, 0))>c\right\}=$ $\left(-2 M, t_{a}\right)$.

In a similar way as in Lemma 4.8 in [38], we define $\hat{\phi}_{\alpha}(i(a)):=\left(\phi_{\alpha}(a), 0, \ldots, 0\right)$ with $\phi_{\alpha}(a):=t_{a}$ and

$$
\mathcal{E}_{\alpha}=\mathcal{E}_{\alpha, c}:=\left\{(i(a) \cdot(t, 0, \ldots, 0)) \in \hat{\mathcal{O}} \cdot \mathbf{V}: f_{\alpha}(i(a) \cdot(t, 0, \ldots, 0))>c\right\} .
$$

Then $\phi_{\alpha}: \mathcal{O} \rightarrow[-2 M, 2 M]$ and

$$
\mathcal{E}_{\alpha} \cap \mathcal{A}^{\prime \prime}=\mathcal{E}_{\hat{\phi}_{\alpha}} \cap \mathcal{A}^{\prime \prime},
$$

where $\mathcal{A}^{\prime \prime}:=\left\{p \in \hat{\mathcal{O}} \cdot \mathbf{V}: p_{1} \in[-2 M, 2 M]\right\}$. Moreover recalling that $c \in(0,1)$, from (52) and (53) we get

$$
S_{\alpha}=\operatorname{graph}\left(\hat{\phi}_{\alpha}\right)=\partial \mathcal{E}_{\alpha} \cap(\hat{\mathcal{O}} \cdot \mathbf{V})
$$

and by Theorem $2.13 \hat{\phi}_{\alpha}$ is uniformly intrinsic differentiable in $\hat{\mathcal{O}}$.

Let $\tilde{f}_{\alpha}: \mathbf{R}^{m+n} \rightarrow \mathbf{R}$ be defined by

$$
\tilde{f}_{\alpha}(t, a):=f_{\alpha}(i(a) \cdot(t, 0, \ldots, 0)) .
$$

By $f_{\alpha} \in \mathbf{C}^{\infty}(\mathbf{G}, \mathbf{R})$ and $(54), \tilde{f}_{\alpha}$ is $\mathbf{C}^{\infty}$ map such that $\frac{\partial \tilde{f}_{\alpha}}{\partial p_{1}}(p)=X_{1} f_{\alpha}(p) \neq 0$ for all $p \in S_{\alpha}$. Hence we apply the classical Implicit Function Theorem to $\tilde{f}_{\alpha}$ and we conclude that also $\hat{\phi}_{\alpha}$ is $\mathbf{C}^{\infty}$.

Step 4. Firstly we prove that $\left(\hat{\phi}_{\alpha}\right)_{\alpha}$, up to subsequence, converges uniformly on $\hat{\mathcal{O}}$, i.e. the condition (1) of the thesis follows. In particular, we would like to show that there is a constant $L>0$ dependent on $\left\|\nabla^{\hat{\phi}} \hat{\phi}\right\|_{\mathcal{L}^{\infty}\left(\hat{\mathcal{O}}, \mathbf{R}^{m-1}\right)}$ (which is finite by Proposition 5.3), $\mathcal{B}^{(1)}, \ldots, \mathcal{B}^{(n)}$ such that for all $\alpha>0$ sufficiently small and all $a \in \mathcal{O}$, it holds

$$
\left|\phi_{\alpha}(a)-\phi(a)\right| \leq L \alpha .
$$

Therefore $\left(\phi_{\alpha}\right)_{\alpha}$, up to subsequence, converges uniformly on $\mathcal{O}$. This is a direct consequence of Theorem 4.1. Here $\nu^{\perp}(p)=\mathbf{P}_{\mathbf{W}}(p), \nu(p)=\mathbf{P}_{\mathbf{V}}(p)$ and $E:=\mathcal{E}_{\hat{\phi}_{\alpha}}$ is the intrinsic subgraph of $\hat{\phi}_{\alpha}$. Because $\hat{\phi}_{\alpha}$ is locally intrinsic Lipschitz, $\mathcal{E}_{\hat{\phi}_{\alpha}}$ is also a finite G-perimeter set (see Theorem 5.1).

For $\beta$ fixed as in Theorem 4.1 applied to $E=\mathcal{E}_{\hat{\phi}_{\alpha}}$ there is $L=L\left(\beta,\|\mathcal{B}\|_{\infty}\right)>0$, where $\|\mathcal{B}\|_{\infty}=\max \left\{b_{i j}^{(s)} \mid i, j=1, \ldots, m, s=1, \ldots, n\right\}$ such that

$$
\mathcal{U}((-L, 0, \ldots, 0), 1) \subset\left\{q:\left\|\mathbf{P}_{\mathbf{W}} q\right\|<-\beta \mathbf{P}_{\mathbf{V}} q\right\}
$$

and

$$
\mathcal{U}((L, 0, \ldots, 0), 1) \subset\left\{q:\left\|\mathbf{P}_{\mathbf{w}} q\right\|<\beta \mathbf{P}_{\mathbf{V}} q\right\} .
$$

Let $p_{a}:=i(a) \cdot \hat{\phi}(i(a)), p_{a}^{\prime}:=i(a) \cdot(\phi(a)-\alpha L, 0, \ldots, 0)$ and $p_{a}^{\prime \prime}:=i(a) \cdot(\phi(a)+$ $\alpha L, 0, \ldots, 0)$ with $a \in \mathcal{O}$ and $\alpha \in(0,1]$. Notice that if we put $r_{0}:=M+L+$ 
$\max _{(x, y) \in \mathcal{O}}|x|_{\mathbf{R}^{m-1}}+\max _{(x, y) \in \mathcal{O}}|y|_{\mathbf{R}^{n}}^{1 / 2}+\frac{1}{2}\|\mathcal{B}\|_{\infty}^{1 / 2} \max _{(x, y) \in \mathcal{O}}|x|_{\mathbf{R}^{m-1}}^{1 / 2}(M+L)^{1 / 2}<+\infty$ then

$$
\left\|p_{a}^{\prime}\right\| \leq r_{0}, \quad\left\|p_{a}^{\prime \prime}\right\| \leq r_{0}
$$

for all $a \in \mathcal{O}$. Moreover, by standard considerations (see [25]) we know that for every $q \in \mathcal{U}\left(0, r_{0}\right)$ there exists $c\left(r_{0}\right)=c\left(r_{0},\|\mathcal{B}\|_{\infty}\right)>0$ such that

$$
\mathcal{U}(0, r) \cdot q \subset \mathcal{U}\left(q, c\left(r_{0}\right) \sqrt{r}\right), \quad \forall r \in(0,1) .
$$

So if $a \in \mathcal{O}$ and $\alpha \in(0,1)$ is small enough, depending on $r_{0}$, then

$$
\begin{aligned}
& \mathcal{U}(0, \alpha) \cdot p_{a}^{\prime} \subset \mathcal{U}\left(p_{a}^{\prime}, c\left(r_{0}\right) \sqrt{\alpha}\right) \subset\left\{q:\left\|\mathbf{P}_{\mathbf{W}}\left(p_{a}^{-1} q\right)\right\|<-\beta\left\|\mathbf{P}_{\mathbf{V}}\left(p_{a}^{-1} q\right)\right\|\right\} \subset \mathcal{E}_{\hat{\phi}_{\alpha}}, \\
& \mathcal{U}(0, \alpha) \cdot p_{a}^{\prime \prime} \subset \mathcal{U}\left(p_{a}^{\prime \prime}, c\left(r_{0}\right) \sqrt{\alpha}\right) \subset\left\{q:\left\|\mathbf{P}_{\mathbf{W}}\left(p_{a}^{-1} q\right)\right\|<\beta\left\|\mathbf{P}_{\mathbf{V}}\left(p_{a}^{-1} q\right)\right\|\right\} \subset \mathbf{G}-\mathcal{E}_{\hat{\phi}_{\alpha}} .
\end{aligned}
$$

More precisely, by definition of $f_{\alpha}$ we have

$$
f_{\alpha}\left(p_{a}^{\prime}\right)=1, \quad f_{\alpha}\left(i(a) \cdot \hat{\phi}_{\alpha}(i(a))\right)=c, \quad f_{\alpha}\left(p_{a}^{\prime \prime}\right)=0,
$$

and so by (58), (59) and (54) we deduce

$$
\phi(a)-\alpha L \leq \phi_{\alpha}(a) \leq \phi(a)+\alpha L
$$

for all $\alpha \in(0,1]$ small enough and for every $a \in \mathcal{O}$. Hence (60) holds and consequently the condition (1) of the thesis is true.

Step 5. We prove that $\left(\hat{\phi}_{\alpha}\right)_{\alpha}$ has a subsequence $\left(\hat{\phi}_{k}\right)_{k}$ such that $\left|\nabla^{\hat{\phi}_{k}} \hat{\phi}_{k}\right|_{\mathbf{R}^{m-1}} \leq$ $\left\|\nabla^{\hat{\phi}} \hat{\phi}\right\|_{\mathcal{L}^{\infty}\left(\hat{\mathcal{O}}, \mathbf{R}^{m-1}\right)}$ for each $k \in \mathbf{N}$ on $\mathcal{O}$, i.e. the condition (2) of the thesis holds.

Let $\hat{\nabla}_{\mathbf{G}} f_{\alpha}:=\left(X_{2} f_{\alpha}, \ldots, X_{m} f_{\alpha}\right)$ and $\hat{\nu}_{\mathcal{E}}=\left(\nu_{\mathcal{E}}^{(2)}, \ldots, \nu_{\mathcal{E}}^{(m)}\right)$. Arguing as in Step 2 and by (15), it follows

$$
\begin{aligned}
\left|\nabla^{\hat{\phi}_{\alpha}} \hat{\phi}_{\alpha}(p)\right|_{\mathbf{R}^{m-1}} & =\frac{\left|\hat{\nabla}_{\mathbf{G}} f_{\alpha}(p)\right|_{\mathbf{R}^{m-1}}}{\left|X_{1} f_{\alpha}(p)\right|} \leq \frac{1}{\left|X_{1} f_{\alpha}(p)\right|} \int_{\mathcal{U}(0, \alpha) \cdot p}\left|\hat{\nu}_{\mathcal{E}}(q) \| \rho_{\alpha}\left(p \cdot q^{-1}\right)\right| d|\partial \mathcal{E}|(q) \\
& \leq I_{\alpha}(p) \frac{\left\|\nabla^{\hat{\phi}} \hat{\phi}\right\|_{\mathcal{L}^{\infty}\left(\hat{\mathcal{O}}, \mathbf{R}^{m-1}\right)}}{\left|X_{1} f_{\alpha}(p)\right| \sqrt{1+\|\nabla \hat{\phi} \hat{\phi}\|_{\mathcal{L}^{\infty}\left(\hat{\mathcal{O}}, \mathbf{R}^{m-1}\right)}^{2}}}
\end{aligned}
$$

and consequently by (56)

$$
\left|\nabla^{\hat{\phi}_{\alpha}} \hat{\phi}_{\alpha}(p)\right|_{\mathbf{R}^{m-1}} \leq\left\|\nabla^{\hat{\phi}} \hat{\phi}\right\|_{\mathcal{L}^{\infty}\left(\hat{\mathcal{O}}, \mathbf{R}^{m-1}\right)},
$$

for all $p \in \hat{\mathcal{O}}$, as desired.

Step 6 . Now we show that $\left(\hat{\phi}_{\alpha}\right)_{\alpha}$, up to a subsequence, satisfying the condition (3) of the thesis, i.e. there exists a sequence $\left(\alpha_{h}\right)_{h} \subset(0,+\infty)$ such that letting $\hat{\phi}_{h} \equiv \hat{\phi}_{\alpha_{h}}$ we have

$$
\nabla^{\hat{\phi}_{h}} \hat{\phi}_{h} \rightarrow \nabla^{\hat{\phi}} \hat{\phi} \quad \text { a.e. in } \hat{\mathcal{O}} \text {. }
$$

It is sufficient to show that there is a positive sequence $\left(\alpha_{h}\right)_{h}$ converging to 0 such that there exists

$$
\lim _{h \rightarrow \infty} \int_{\mathcal{O}} \sqrt{1+\left|\nabla^{\hat{\phi}_{h}} \hat{\phi}_{h}\right|_{\mathbf{R}^{m-1}}^{2}} d \mathcal{L}^{m+n-1}=\int_{\mathcal{O}} \sqrt{1+\left|\nabla^{\hat{\phi}} \hat{\phi}\right|_{\mathbf{R}^{m-1}}^{2}} d \mathcal{L}^{m+n-1}
$$

In fact, up a subsequence, using (61) and Proposition 5.6 we can assume that the sequence in (63) also satisfies

$$
\nabla^{\hat{\phi}_{h}} \hat{\phi}_{h} \rightarrow \nabla^{\hat{\phi}} \hat{\phi} \quad \text { weakly in } \mathcal{L}^{1}\left(\hat{\mathcal{O}}, \mathbf{R}^{m-1}\right) .
$$


Consequently, by Theorem 6.4

$$
\nabla^{\hat{\phi}_{h}} \hat{\phi}_{h} \rightarrow \nabla^{\hat{\phi}} \hat{\phi} \quad \text { strongly in } \mathcal{L}^{1}\left(\hat{\mathcal{O}}, \mathbf{R}^{m-1}\right)
$$

and so, up a subsequence, (62) holds.

In order to show (63) we need to prove that there exist $\hat{c} \in(0,1)$ and a positive sequence $\left(\alpha_{h}\right)_{h}$ converging to 0 satisfying

$$
\exists \lim _{h \rightarrow \infty}\left|\partial \mathcal{E}_{\alpha_{h}, \hat{c}}\right|_{\mathbf{G}}(\mathcal{A})=|\partial \mathcal{E}|_{\mathbf{G}}(\mathcal{A})
$$

where $\mathcal{A}$ is defined as in Step 2. By the semicontinuity of G-perimeter measure and Step 3 we obtain

$$
|\partial \mathcal{E}|_{\mathbf{G}}(\mathcal{A}) \leq \liminf _{\alpha \rightarrow 0^{+}}\left|\partial \mathcal{E}_{\alpha, c}\right|_{\mathbf{G}}(\mathcal{A})
$$

for every $c \in(0,1)$. More precisely, by the last inequality and the coarea formula we get

$$
\begin{aligned}
|\partial \mathcal{E}|_{\mathbf{G}}(\mathcal{A}) & \leq \int_{0}^{1} \liminf _{\alpha \rightarrow 0^{+}}\left|\partial \mathcal{E}_{\alpha, c}\right|_{\mathbf{G}}(\mathcal{A}) d c \leq \liminf _{\alpha \rightarrow 0^{+}} \int_{0}^{1}\left|\partial \mathcal{E}_{\alpha, c}\right|_{\mathbf{G}}(\mathcal{A}) d c \\
& =\liminf _{\alpha \rightarrow 0^{+}} \int_{\mathcal{A}}\left|\nabla_{\mathbf{G}} f_{\alpha}\right| d \mathcal{L}^{m+n}=: I(\hat{\mathcal{O}}, c) .
\end{aligned}
$$

Now using Step 2 we know that for every $\hat{\mathcal{O}}_{0}$ such that $\hat{\mathcal{O}} \Subset \hat{\mathcal{O}}_{0}$ open and bounded there exists $\left(\alpha_{h}\right)_{h} \subset(0,+\infty)$ which converges to 0 and $\bar{h}\left(\hat{\mathcal{O}}_{0}\right)>0$ such that for all $h \leq \bar{h}\left(\hat{\mathcal{O}}_{0}\right)$ we have

$$
\int_{\mathcal{A}}\left|\nabla_{\mathbf{G}} f_{\alpha_{h}}\right| d \mathcal{L}^{m+n} \leq|\partial \mathcal{E}|_{\mathbf{G}}\left(\mathcal{A}_{0}\right)
$$

where $\mathcal{A}, \mathcal{A}_{0}$ are defined as in Step 2. Consequently,

$$
I(\hat{\mathcal{O}}, c) \leq|\partial \mathcal{E}|_{\mathbf{G}}\left(\mathcal{A}_{0}\right)
$$

for all $c \in(0,1)$ and $\hat{\mathcal{O}}_{0}$ such that $\hat{\mathcal{O}} \Subset \hat{\mathcal{O}}_{0}$ open and bounded. Moreover since $|\partial \mathcal{E}|_{\text {G }}$ is a Radon measure then by a standard approximation argument we can rewrite (66) with $\hat{\mathcal{O}}$ instead of $\hat{\mathcal{O}}_{0}$. In particular using $(65)$ we have that a.e. $c \in(0,1)$

$$
\liminf _{\alpha \rightarrow 0^{+}}\left|\partial \mathcal{E}_{\alpha, c}\right|_{\mathbf{G}}(\mathcal{A})=|\partial \mathcal{E}|_{\mathbf{G}}(\mathcal{A}) .
$$

Hence there exists $\hat{c} \in(0,1)$ and a positive sequence $\left(\alpha_{h}\right)_{h}$ converging to 0 such that (64) follows.

Finally, because $\|\phi\|_{\mathcal{L}^{\infty}(\mathcal{O}, \mathbf{R})}=M$ we get

$$
\partial \mathcal{E} \cap \mathcal{A}_{1}=\partial \mathcal{E} \cap \mathcal{A}_{2}=\emptyset
$$

where $\mathcal{A}_{1}:=\left\{p \in \hat{\mathcal{O}} \cdot \mathbf{V}: p_{1} \leq-2 M\right\}$ and $\mathcal{A}_{2}:=\left\{p \in \hat{\mathcal{O}} \cdot \mathbf{V}: p_{1} \geq 2 M\right\}$.

Hence by Proposition 5.7 and well-know G-perimeter properties it follows that

$$
\begin{aligned}
& \frac{1}{C(\mathbf{W}, \mathbf{V})} \int_{\mathcal{O}} \sqrt{1+|\nabla \hat{\phi} \hat{\phi}|_{\mathbf{R}^{m-1}}^{2}} d \mathcal{L}^{m+n-1} \\
& =|\partial \mathcal{E}|_{\mathbf{G}}(\hat{\mathcal{O}} \cdot \mathbf{V})=|\partial \mathcal{E}|_{\mathbf{G}}\left(\mathcal{A}_{1}\right)+|\partial \mathcal{E}|_{\mathbf{G}}(\mathcal{A})+|\partial \mathcal{E}|\left(\mathcal{A}_{2}\right) \\
& =|\partial \mathcal{E}|_{\mathbf{G}}\left(\partial \mathcal{E} \cap \mathcal{A}_{1}\right)+|\partial \mathcal{E}|_{\mathbf{G}}(\mathcal{A})+|\partial \mathcal{E}|_{\mathbf{G}}\left(\partial \mathcal{E} \cap \mathcal{A}_{2}\right)=|\partial \mathcal{E}|_{\mathbf{G}}(\mathcal{A}) .
\end{aligned}
$$

In the same way, using also (52), (53) and (58)

and

$$
\left|\partial \mathcal{E}_{\alpha_{h}, c}\right|_{\mathbf{G}}(\mathcal{A})=\left|\partial \mathcal{E}_{\hat{\phi}_{h}}\right|_{\mathbf{G}}(\mathcal{A})
$$

$$
\left|\partial \mathcal{E}_{\hat{\phi}_{h}}\right|_{\mathbf{G}}(\hat{\mathcal{O}} \cdot \mathbf{V})=\frac{1}{C(\mathbf{W}, \mathbf{V})} \int_{\mathcal{O}} \sqrt{1+\left|\nabla^{\hat{\phi}_{h}} \hat{\phi}_{h}\right|_{\mathbf{R}^{m-1}}^{2}} d \mathcal{L}^{m+n-1}
$$


with $\hat{\phi}_{h}=\hat{\phi}_{\alpha_{h}}$. Now by the last three equalities and (64), (63) holds. Consequently, (62) is true and the proof of theorem is complete.

\section{Intrinsic Lipschitz function and distributional solution}

The main results in this section are Theorem 7.1 and Theorem 7.2. We show that a map $\hat{\phi}=(\phi, 0 \ldots, 0): \hat{\mathcal{O}} \rightarrow \mathbf{V}$ is a locally intrinsic Lipschitz function if and only if $\phi$ is locally $1 / 2$-Hölder continuous and it is also distributional solution of system

$$
\left(D_{2}^{\phi} \phi, \ldots, D_{m}^{\phi} \phi\right)=w \quad \text { in } \mathcal{O}
$$

for $w \in \mathcal{L}_{\text {loc }}^{\infty}\left(\mathcal{O}, \mathbf{R}^{m-1}\right)$. This result is well known in Heisenberg groups (see Theorem 1.1 in [5]). More precisely in [5], the authors omit the little Hölder continuity of $\phi$ because the other assumption (i.e. $\phi$ is distributional solution of system $\left(D_{2}^{\phi} \phi, \ldots, D_{m}^{\phi} \phi\right)=w$ in $\left.\mathcal{O}\right)$ implies it. We use this hypothesis in the proof of Lemma 7.3, but it is not clear if we can omit it.

Theorem 7.1. Let $\mathbf{G}:=\left(\mathbf{R}^{m+n}, \cdot, \delta_{\lambda}\right)$ be a Carnot group of step 2 and $\mathbf{V}, \mathbf{W}$ the complementary subgroups defined in (13). Let $\hat{\phi}: \hat{\mathcal{O}} \rightarrow \mathbf{V}$ be a locally intrinsic Lipschitz function, where $\hat{\mathcal{O}}$ is an open subset of $\mathbf{W}$ and $\phi: \mathcal{O} \rightarrow \mathbf{R}$ is the map associated to $\hat{\phi}$ as in (14). Then $\phi$ is a distributional solution of $\left(D_{2}^{\phi} \phi, \ldots, D_{m}^{\phi} \phi\right)=w$ for a suitable $w \in \mathcal{L}_{\text {loc }}^{\infty}\left(\mathcal{O}, \mathbf{R}^{m-1}\right)$ such that $w(a)=\left(D_{2}^{\phi} \phi(a), \ldots, D_{m}^{\phi} \phi(a)\right) \mathcal{L}^{m+n-1}$ a.e. in $\mathcal{O}$.

Proof. By Theorem 6.1 it is immediate the fact that there exists $\left(\phi_{h}\right)_{h \in \mathbf{N}} \subset$ $\mathbf{C}^{\infty}(\mathcal{O}, \mathbf{R})$ such that for all $\mathcal{O}^{\prime} \Subset \mathcal{O}$ we have

(1) $\hat{\phi}_{h}$ uniformly converges to $\hat{\phi}$ in $\hat{\mathcal{O}}^{\prime}$,

(2) $\left|\nabla^{\hat{\phi}_{h}} \hat{\phi}_{h}(a)\right| \leq\left\|\nabla^{\hat{\phi}} \hat{\phi}\right\|_{\mathcal{L}^{\infty}\left(\hat{\mathcal{O}}^{\prime}, \mathbf{R}^{m-1}\right)} \forall a \in \hat{\mathcal{O}}^{\prime}, h \in \mathbf{N}$,

(3) $\nabla^{\hat{\phi}_{h}} \hat{\phi}_{h}(a) \rightarrow \nabla^{\hat{\phi}} \hat{\phi}(a)$ a.e. $a \in \hat{\mathcal{O}}^{\prime}$.

Let $w_{h}=\left(w_{2, h}, \ldots, w_{m, h}\right):=\nabla^{\hat{\phi}_{h}} \hat{\phi}_{h}$. Observe that for each $h \in \mathbf{N}$ and $\zeta \in$ $\mathbf{C}_{c}^{\infty}(\mathcal{O}, \mathbf{R})$

$$
\int_{\mathcal{O}} \phi_{h}\left(X_{j} \zeta+\phi_{h} \sum_{s=1}^{n} b_{j 1}^{(s)} Y_{s} \zeta\right) d \mathcal{L}^{m+n-1}=-\int_{\mathcal{O}} w_{j, h} \zeta d \mathcal{L}^{m+n-1}
$$

for all $j=2, \ldots, m$. Getting to the limit for $h \rightarrow \infty$ we have

$$
\int_{\mathcal{O}} \phi\left(X_{j} \zeta+\phi \sum_{s=1}^{n} b_{j 1}^{(s)} Y_{s} \zeta\right) d \mathcal{L}^{m+n-1}=-\int_{\mathcal{O}} w_{j} \zeta d \mathcal{L}^{m+n-1} .
$$

Hence $\phi$ is a distributional solution of $\left(D_{2}^{\phi} \phi, \ldots, D_{m}^{\phi} \phi\right)=w$ in $\mathcal{O}$.

Theorem 7.2. Let $\mathbf{G}:=\left(\mathbf{R}^{m+n}, \cdot, \delta_{\lambda}\right)$ be a Carnot group of step 2 and $\mathbf{V}, \mathbf{W}$ the complementary subgroups defined in (13). Let $\hat{\phi}: \hat{\mathcal{O}} \rightarrow \mathbf{V}$ be a continuous map where $\hat{\mathcal{O}}$ is open in $\mathbf{W}$ and $\phi: \mathcal{O} \rightarrow \mathbf{R}$ is the map associated to $\hat{\phi}$ as in (14). We also assume that

(1) $\phi$ is a continuous distributional solution of $\left(D_{2}^{\phi} \phi, \ldots, D_{m}^{\phi} \phi\right)=w$ in $\mathcal{O}$ with $w \in \mathcal{L}_{\mathrm{loc}}^{\infty}\left(\mathcal{O}, \mathbf{R}^{m-1}\right)$

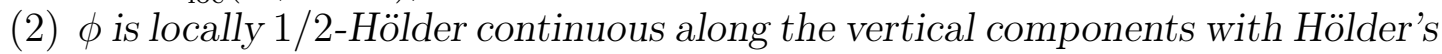
constant $C_{h}>0$.

Then $\hat{\phi}$ is locally intrinsic Lipschitz function. 
The proof of Theorem 7.2 relies on a preliminary result about the distributional solutions of the problem $\left(D_{2}^{\phi} \phi, \ldots, D_{m}^{\phi} \phi\right)=w$ in $\mathcal{O}$.

It is convenient to introduce the following notation: for $j=2, \ldots, m$ and $x=$ $\left(x_{2}, \ldots, x_{m}\right) \in \mathbf{R}^{m-1}$ fixed, we denote by $\left(t, \hat{x}_{j}\right):=\left(x_{2}, \ldots, x_{j-1}, t, x_{j+1}, \ldots, x_{m}\right)$.

Lemma 7.3. Under the same assumptions of Theorem 7.2, for all $j=2, \ldots, m$ there is $C=C\left(\left\|w_{j}\right\|_{\mathcal{L}^{\infty}(\mathcal{O}, \mathbf{R})}, C_{h}, b_{j 1}^{(1)}, \ldots, b_{j 1}^{(n)}\right)>0$ such that $t \mapsto \phi\left(t, \hat{x}_{j}, \gamma_{j}(t)\right)$ is a C-Lipschitz map along any characteristic line $\gamma_{j}=\left(\gamma_{j 1}, \ldots, \gamma_{j n}\right):[-T, T] \rightarrow \mathbf{R}^{n}$ satisfying

$$
\dot{\gamma}_{j s}(t)= \begin{cases}b_{j 1}^{(s)} \phi\left(t, \hat{x}_{j}, \gamma_{j}(t)\right)+\frac{1}{2} \sum_{\substack{l=2 \\ l \neq j}}^{m} b_{j l}^{(s)} x_{l} & \text { if } m>2, \text { for all } s=1, \ldots, n, \\ b_{j 1}^{(s)} \phi\left(t, \gamma_{j}(t)\right) & \text { if } m=2, \text { for all } s=1, \ldots, n,\end{cases}
$$

with $t \in[-T, T]$ and $\hat{x}_{j} \in \mathbf{R}^{m-2}$ fixed.

We compute the characteristic lines inside the free step 2 group $\mathbf{F}_{m, 2}$ (see Remark 3.1).

Example 7.1. We consider the free step 2 group $\mathbf{F}_{m, 2}$. As we said in Remark 3.1, the composition law (10) is given by $\mathcal{B}^{(s)} \equiv \mathcal{B}^{(l, h)}$ where $1 \leq h<l \leq m$ and $\mathcal{B}^{(l, h)}$ has entries -1 in position $(l, h), 1$ in position $(h, l)$ and 0 everywhere else.

For each $j=2, \ldots, m$ and $\hat{x}_{j} \in \mathbf{R}^{m-2}$ fixed, the characteristic line $\gamma_{j}=\left(\gamma_{j 1}, \ldots\right.$, $\left.\gamma_{j n}\right):[-T, T] \rightarrow \mathbf{R}^{n}$ has the following form:

$$
\begin{aligned}
& \dot{\gamma}_{j s_{1}}(t)=b_{j 1}^{\left(s_{1}\right)} \phi\left(t, \hat{x}_{j}, \gamma_{j}(t)\right)=-\phi\left(t, \hat{x}_{j}, \gamma_{j}(t)\right), \\
& \dot{\gamma}_{j s_{2}}(t)=\frac{1}{2} b_{j h_{2}}^{\left(s_{2}\right)} x_{h_{2}}, \\
& \dot{\gamma}_{j s_{3}}(t)=0 .
\end{aligned}
$$

where $\mathcal{B}^{\left(s_{i}\right)} \equiv \mathcal{B}^{\left(l_{i}, h_{i}\right)}$ is such that

- $s_{1}$ is the unique index $\left(l_{1}, h_{1}\right)=(j, 1)$,

- $s_{2}$ is such that $h_{2}=j$ and $j<l_{2} \leq m$ or such that $l_{2}=j$ and $1<h_{2}<j$,

- $s_{3}$ represents the other cases.

Now we show the proof of Lemma 7.3.

Proof. Fix $j=3, \ldots, m$ and $\hat{x}_{j} \in \mathbf{R}^{m-2}$. For simplicity we just consider the case $j>2$, but the proof in the case $j=2$ is exactly the same because the term $\frac{1}{2} \sum_{\substack{l=2 \\ l \neq j}}^{m} b_{j l}^{(s)} x_{l}$ does not affect the accounts.

Let $t_{1}, t_{2} \in(-T, T)$ with $t_{1}<t_{2}, \epsilon>0$ and $r_{s}$ is a non negative constant such that $r_{s} \geq \max \left\{\left|\min _{x} \gamma_{j s}(x)\right|,\left|\max _{x} \gamma_{j s}(x)\right|\right\}$ for $s=1, \ldots, n$. We would like to prove that there is $C>0$ such that

$$
\left|\phi\left(t_{2}, \hat{x}_{j}, \gamma_{j}\left(t_{2}\right)\right)-\phi\left(t_{1}, \hat{x}_{j}, \gamma_{j}\left(t_{1}\right)\right)\right| \leq C\left(t_{2}-t_{1}\right),
$$

for $t_{1}, t_{2} \in(-T, T)$ with $t_{1}<t_{2}$.

If $\phi$ is distributional solution of $\left(D_{2}^{\phi} \phi, \ldots, D_{m}^{\phi} \phi\right)=w$ in $\mathcal{O}$, then for all test functions $\varphi$ with $\operatorname{supp}(\varphi) \Subset(-T, T) \times\left(-r_{1}, r_{1}\right) \times \cdots \times\left(-r_{n}, r_{n}\right)$ we have

$$
\begin{aligned}
\int_{-T}^{T} \int_{-r_{1}}^{r_{1}} \ldots \int_{-r_{n}}^{r_{n}} & \left(\phi\left(x, \hat{x}_{j}, y\right) \varphi_{x}(x, y)+f_{1}\left(\phi\left(x, \hat{x}_{j}, y\right)\right) \varphi_{y_{1}}(x, y)+\ldots\right. \\
& \left.+f_{n}(\phi(x, y)) \varphi_{y_{n}}(x, y)+w_{j}\left(x, \hat{x}_{j}, y\right) \varphi(x, y)\right) d y d x=0
\end{aligned}
$$


where

$$
f_{s}\left(\phi\left(x_{j}, \hat{x}_{j}, y\right)\right)=\frac{1}{2}\left(b_{j 1}^{(s)} \phi^{2}\left(x_{j}, \hat{x}_{j}, y\right)+\phi\left(x_{j}, \hat{x}_{j}, y\right) \sum_{\substack{l=2 \\ l \neq j}}^{m} b_{j l}^{(s)} x_{l}\right)
$$

for all $\left(x, \hat{x}_{j}, y\right) \in \mathcal{O}$ and $s=1, \ldots, n$.

In a similar way as in [10] and in Lemma 5.1 [5], we choose a test function $\varphi$ which depends on $\delta$ and $\epsilon$; that is $\varphi\left(x, y_{1}, \ldots, y_{n}\right)=\xi_{1}\left(x, y_{1}\right) \ldots \xi_{n}\left(x, y_{n}\right) h(x)$ where the functions $\xi_{1}\left(x, y_{1}\right), \ldots, \xi_{n}\left(x, y_{n}\right)$ and $h(x)$ are defined for small $\delta>0$ by

$$
\xi_{s}\left(x, y_{s}\right)= \begin{cases}0, & -T<x<T,-r_{s}<y_{s} \leq \gamma_{j s}(x)-\epsilon-\delta, \\ \frac{1}{\delta}\left(y_{s}-\gamma_{j s}(x)+\epsilon+\delta\right), & -T<x<T, \gamma_{j s}(x)-\epsilon-\delta<y_{s} \leq \gamma_{j s}(x)-\epsilon, \\ 1, & -T<x<T, \gamma_{j s}(x)-\epsilon<y_{s} \leq \gamma_{j s}(x), \\ \frac{1}{\delta}\left(-y_{s}+\gamma_{j s}(x)+\delta\right), & -T<x<T, \gamma_{j s}(x)<y_{s} \leq \gamma_{j s}(x)+\delta, \\ 0, & -T<x<T, \gamma_{j s}(x)+\delta<y_{s}<r_{s},\end{cases}
$$

for $s=1, \ldots, n$, and

$$
h(x)= \begin{cases}0, & -T<x \leq t_{1}-\delta \\ \frac{1}{\delta}\left(x-t_{1}+\delta\right), & t_{1}-\delta<x \leq t_{1} \\ 1, & t_{1}<x \leq t_{2} \\ \frac{1}{\delta}\left(-x+t_{2}+\delta\right), & t_{2}<x \leq t_{2}+\delta \\ 0, & t_{2}+\delta<x<T\end{cases}
$$

We compute the limit $\delta \rightarrow 0^{+}$:

$$
\begin{aligned}
0=\lim _{\delta \rightarrow 0^{+}} & \int_{-T}^{T} \int_{-r_{1}}^{r_{1}} \ldots \int_{-r_{n}}^{r_{n}}\left[\phi\left(x, \hat{x}_{j}, y\right)\left(\xi_{1}\right)_{x}\left(x, y_{1}\right) \xi_{2}\left(x, y_{2}\right) \ldots \xi_{n}\left(x, y_{n}\right) h(x)+\ldots\right. \\
& +\phi\left(x, \hat{x}_{j}, y\right) \xi_{1}\left(x, y_{1}\right) \ldots \xi_{n-1}\left(x, y_{n-1}\right)\left(\xi_{n}\right)_{x}\left(x, y_{n}\right) h(x) \\
& +\phi\left(x, \hat{x}_{j}, y\right) \xi_{1}\left(x, y_{1}\right) \ldots \xi_{n-1}\left(x, y_{n-1}\right) \xi_{n}\left(x, y_{n}\right) h_{x}(x) \\
& +f_{1}\left(\phi\left(x, \hat{x}_{j}, y\right)\right)\left(\xi_{1}\right)_{y_{1}}\left(x, y_{1}\right) \xi_{2}\left(x, y_{2}\right) \ldots \xi_{n}\left(x, y_{n}\right) h(x)+\ldots \\
& +f_{n}\left(\phi\left(x, \hat{x}_{j}, y\right)\right) \xi_{1}\left(x, y_{1}\right) \ldots\left(\xi_{n}\right)_{y_{n}}\left(x, y_{n}\right) h(x) \\
& \left.+w_{j}\left(x, \hat{x}_{j}, y\right) \xi_{1}\left(x, y_{1}\right) \ldots \xi_{n}\left(x, y_{n}\right) h(x)\right] d y d x \\
& =: I_{\varphi_{x} 1}+\cdots+I_{\varphi_{x} n}+I_{\varphi_{x} h}+I_{\varphi_{y} 1}+\cdots+I_{\varphi_{y} n}+I_{w} .
\end{aligned}
$$

Then

$$
\begin{aligned}
\lim _{\delta \rightarrow 0^{+}} I_{\varphi_{x} 1}= & \lim _{\delta \rightarrow 0^{+}} \int_{-T}^{T} \int_{-r_{1}}^{r_{1}} \ldots \int_{-r_{n}}^{r_{n}} \phi\left(\xi_{1}\right)_{x} \xi_{2} \ldots \xi_{n} h d y d x \\
= & \int_{t_{1}}^{t_{2}} \int_{\gamma_{j 2}(x)-\epsilon}^{\gamma_{j 2}(x)} \ldots \int_{\gamma_{j n}(x)-\epsilon}^{\gamma_{j n}(x)} \dot{\gamma}_{j 1}(x)\left(\phi\left(x, \hat{x}_{j}, \gamma_{j 1}(x), y_{2}, \ldots, y_{n}\right)\right. \\
& \left.-\phi\left(x, \hat{x}_{j}, \gamma_{j 1}(x)-\epsilon, y_{2}, \ldots, y_{n}\right)\right) d y_{n} \ldots d y_{2} d x
\end{aligned}
$$




$$
\begin{aligned}
\lim _{\delta \rightarrow 0^{+}} I_{\varphi_{x} n}= & \lim _{\delta \rightarrow 0^{+}} \int_{-T}^{T} \int_{-r_{1}}^{r_{1}} \ldots \int_{-r_{n}}^{r_{n}} \phi \xi_{1} \xi_{2} \ldots\left(\xi_{n}\right)_{x} h d y d x \\
= & \int_{t_{1}}^{t_{2}} \int_{\gamma_{j 1}(x)-\epsilon}^{\gamma_{j 1}(x)} \ldots \int_{\gamma_{j(n-1)}(x)-\epsilon}^{\gamma_{j(n-1)}(x)} \dot{\gamma}_{j n}(x)\left(\phi\left(x, \hat{x}_{j}, y_{1}, \ldots, y_{n-1}, \gamma_{j n}(x)\right)\right. \\
& \left.-\phi\left(x, \hat{x}_{j}, y_{1}, \ldots, y_{n-1}, \gamma_{j n}(x)-\epsilon\right)\right) d y_{n-1} \ldots d y_{1} d x
\end{aligned}
$$

$\lim _{\delta \rightarrow 0^{+}} I_{\varphi_{x} h}=\lim _{\delta \rightarrow 0^{+}} \int_{-T}^{T} \int_{-r_{1}}^{r_{1}} \ldots \int_{-r_{n}}^{r_{n}} \phi \xi_{1} \ldots \xi_{n} h_{x} d y d x$

$$
=\int_{\gamma_{1}\left(t_{1}\right)-\epsilon}^{\gamma_{j 1}\left(t_{1}\right)} \cdots \int_{\gamma_{j n}\left(t_{1}\right)-\epsilon}^{\gamma_{j n}\left(t_{1}\right)} \phi\left(t_{1}, \hat{x}_{j}, y\right) d y-\int_{\gamma_{j 1}\left(t_{2}\right)-\epsilon}^{\gamma_{j 1}\left(t_{2}\right)} \ldots \int_{\gamma_{j n}\left(t_{2}\right)-\epsilon}^{\gamma_{j n}\left(t_{2}\right)} \phi\left(t_{2}, \hat{x}_{j}, y\right) d y
$$

$\lim _{\delta \rightarrow 0^{+}} I_{\varphi_{y} 1}=\lim _{\delta \rightarrow 0^{+}} \int_{-T}^{T} \int_{-r_{1}}^{r_{1}} \ldots \int_{-r_{n}}^{r_{n}} f_{1}(\phi)\left(\xi_{1}\right)_{y_{1}} \xi_{2} \ldots \xi_{n} h d y d x$

$$
\begin{aligned}
= & \int_{t_{1}}^{t_{2}} \int_{\gamma_{j 2}(x)-\epsilon}^{\gamma_{j 2}(x)} \cdots \int_{\gamma_{j n}(x)-\epsilon}^{\gamma_{j n}(x)}\left(f_{1}\left(\phi\left(x, \hat{x}_{j}, \gamma_{j 1}(x)-\epsilon, y_{2}, \ldots, y_{n}\right)\right)\right. \\
& \left.-f_{1}\left(\phi\left(x, \hat{x}_{j}, \gamma_{j 1}(x), y_{2}, \ldots, y_{n}\right)\right)\right) d y_{n} \ldots d y_{2} d x
\end{aligned}
$$

$$
\begin{aligned}
\lim _{\delta \rightarrow 0^{+}} I_{\varphi_{y} n}= & \lim _{\delta \rightarrow 0^{+}} \int_{-T}^{T} \int_{-r_{1}}^{r_{1}} \ldots \int_{-r_{n}}^{r_{n}} f_{n}(\phi) \xi_{1} \ldots \xi_{n-1}\left(\xi_{n}\right)_{y_{n}} h d y d x \\
= & \int_{t_{1}}^{t_{2}} \int_{\gamma_{j 1}(x)-\epsilon}^{\gamma_{j 1}(x)} \ldots \int_{\gamma_{j(n-1)}(x)-\epsilon}^{\gamma_{j(n-1)}(x)}\left(f_{n}\left(\phi\left(x, y_{1}, \ldots, y_{n-1}, \gamma_{j n}(x)-\epsilon\right)\right)\right. \\
& \left.-f_{n}\left(\phi\left(x, y_{1}, \ldots, y_{n-1}, \gamma_{j n}(x)\right)\right)\right) d y_{n-1} \ldots d y_{1} d x
\end{aligned}
$$

$\lim _{\delta \rightarrow 0^{+}} I_{w}=\lim _{\delta \rightarrow 0^{+}} \int_{-T}^{T} \int_{-r_{1}}^{r_{1}} \ldots \int_{-r_{n}}^{r_{n}} w_{j} \xi_{1} \ldots \xi_{n} h d y d x$

$$
=\int_{t_{1}}^{t_{2}} \int_{\gamma_{j 1}(x)-\epsilon}^{\gamma_{j 1}(x)} \cdots \int_{\gamma_{j n}(x)-\epsilon}^{\gamma_{j n}(x)} w_{j}\left(x, \hat{x}_{j}, y\right) d y d x
$$

Hence we obtain

$$
\begin{aligned}
& \int_{\gamma_{j 1}\left(t_{2}\right)-\epsilon}^{\gamma_{j 1}\left(t_{2}\right)} \ldots \int_{\gamma_{j n}\left(t_{2}\right)-\epsilon}^{\gamma_{j n}\left(t_{2}\right)} \phi\left(t_{2}, \hat{x}_{j}, y\right) d y-\int_{\gamma_{j 1}\left(t_{1}\right)-\epsilon}^{\gamma_{j 1}\left(t_{1}\right)} \ldots \int_{\gamma_{j n}\left(t_{1}\right)-\epsilon}^{\gamma_{j n}\left(t_{1}\right)} \phi\left(t_{1}, \hat{x}_{j}, y\right) d y \\
& \quad-\int_{t_{1}}^{t_{2}} \int_{\gamma_{j 1}(x)-\epsilon}^{\gamma_{j 1}(x)} \ldots \int_{\gamma_{j n}(x)-\epsilon}^{\gamma_{j n}(x)} w_{j}\left(x, \hat{x}_{j}, y\right) d y d x \\
& =\int_{t_{1}}^{t_{2}} \int_{\gamma_{j 2}(x)-\epsilon}^{\gamma_{j 2}(x)} \ldots \int_{\gamma_{j n}(x)-\epsilon}^{\gamma_{j n}(x)}\left(f_{1}\left(\phi\left(x, \hat{x}_{j}, \gamma_{j 1}(x)-\epsilon, y_{2}, \ldots, y_{n}\right)\right)\right. \\
& \left.\quad-f_{1}\left(\phi\left(x, \hat{x}_{j}, \gamma_{j 1}(x), y_{2}, \ldots, y_{n}\right)\right)\right)+\dot{\gamma}_{j 1}(x)\left(\phi\left(x, \hat{x}_{j}, \gamma_{j 1}(x), y_{2}, \ldots, y_{n}\right)\right. \\
& \left.\quad-\phi\left(x, \hat{x}_{j}, \gamma_{j 1}(x)-\epsilon, y_{2}, \ldots, y_{n}\right)\right) d y_{n} \ldots d y_{2} d x+\cdots
\end{aligned}
$$




$$
\begin{aligned}
& +\int_{t_{1}}^{t_{2}} \int_{\gamma_{j 1}(x)-\epsilon}^{\gamma_{j 1}(x)} \ldots \int_{\gamma_{j(n-1)}(x)-\epsilon}^{\gamma_{j(n-1)}(x)}\left(f_{n}\left(\phi\left(x, \hat{x}_{j}, y_{1}, \ldots, y_{n-1}, \gamma_{j n}(x)-\epsilon\right)\right)\right. \\
& \left.-f_{n}\left(\phi\left(x, \hat{x}_{j}, y_{1}, \ldots, y_{n-1}, \gamma_{j n}(x)\right)\right)\right)+\dot{\gamma}_{j n}(x)\left(\phi\left(x, \hat{x}_{j}, y_{1}, \ldots, y_{n-1}, \gamma_{j n}(x)\right)\right. \\
& \left.-\phi\left(x, \hat{x}_{j}, y_{1}, \ldots, y_{n-1}, \gamma_{j n}(x)-\epsilon\right)\right) d y_{n-1} \ldots d y_{1} d x
\end{aligned}
$$

and, consequently, by (68) we deduce that

$$
\begin{aligned}
& \int_{\gamma_{j 1}\left(t_{2}\right)-\epsilon}^{\gamma_{j 1}\left(t_{2}\right)} \ldots \int_{\gamma_{j n}\left(t_{2}\right)-\epsilon}^{\gamma_{j n}\left(t_{2}\right)} \phi\left(t_{2}, \hat{x}_{j}, y\right) d y-\int_{\gamma_{j 1}\left(t_{1}\right)-\epsilon}^{\gamma_{j 1}\left(t_{1}\right)} \ldots \int_{\gamma_{j n}\left(t_{1}\right)-\epsilon}^{\gamma_{j n}\left(t_{1}\right)} \phi\left(t_{1}, \hat{x}_{j}, y\right) d y \\
& \quad-\int_{t_{1}}^{t_{2}} \int_{\gamma_{j 1}(x)-\epsilon}^{\gamma_{j 1}(x)} \ldots \int_{\gamma_{j n}(x)-\epsilon}^{\gamma_{j n}(x)} w_{j}\left(x, \hat{x}_{j}, y\right) d y d x \\
& =\int_{t_{1}}^{t_{2}} \int_{\gamma_{j 2}(x)-\epsilon}^{\gamma_{j 2}(x)} \ldots \int_{\gamma_{j n}(x)-\epsilon}^{\gamma_{j n}(x)} \frac{1}{2} b_{j 1}^{(1)}\left(\phi\left(x, \hat{x}_{j}, \gamma_{j 1}(x)-\epsilon, y_{2}, \ldots, y_{n}\right)\right. \\
& \left.\quad-\phi\left(x, \hat{x}_{j}, \gamma_{j 1}(x), y_{2}, \ldots, y_{n}\right)\right)\left(\phi\left(x, \hat{x}_{j}, \gamma_{j 1}(x)-\epsilon, y_{2}, \ldots, y_{n}\right)\right. \\
& \left.\quad+\phi\left(x, \hat{x}_{j}, \gamma_{j 1}(x), y_{2}, \ldots, y_{n}\right)-2 \phi\left(x, \hat{x}_{j}, \gamma_{j}(x)\right)\right) d y_{n} \ldots d y_{2} d x+\ldots \\
& \quad+\int_{t_{1}}^{t_{2}} \int_{\gamma_{j 1}(x)-\epsilon}^{\gamma_{j 1}(x)} \ldots \int_{\gamma_{j(n-1)}(x)-\epsilon}^{\gamma_{j(n-1)}(x)} \frac{1}{2} b_{j 1}^{(1)}\left(\phi\left(x, \hat{x}_{j}, y_{1}, \ldots, y_{n-1}, \gamma_{j n}(x)-\epsilon\right)\right. \\
& \left.\quad-\phi\left(x, \hat{x}_{j}, y_{1}, \ldots, y_{n-1}, \gamma_{j n}(x)\right)\right)\left(\phi\left(x, \hat{x}_{j}, y_{1}, \ldots, y_{n-1}, \gamma_{j n}(x)-\epsilon\right)\right. \\
& \left.\left.\quad+\phi\left(x, \hat{x}_{j}, y_{1}, \ldots, y_{n-1}, \gamma_{j n}(x)\right)\right)-2 \phi\left(x, \hat{x}_{j}, \gamma_{j}(x)\right)\right) d y_{n-1} \ldots d y_{1} d x .
\end{aligned}
$$

Because $\phi$ is locally 1/2-Hölder continuous along the vertical components with Hölder's constant $C_{h}>0$, we have

$$
\begin{aligned}
\frac{\left|\phi\left(x, \hat{x}_{j}, \gamma_{j 1}(x)-\epsilon, y_{2}, \ldots, y_{n}\right)-\phi\left(x, \hat{x}_{j}, \gamma_{j 1}(x), y_{2}, \ldots, y_{n}\right)\right|}{\sqrt{\epsilon}} & \leq C_{h}, \\
\frac{\left|\phi\left(x, \hat{x}_{j}, \gamma_{j 1}(x)-\epsilon, y_{2}, \ldots, y_{n}\right)-\phi\left(x, \hat{x}_{j}, \gamma_{j}(x)\right)\right|}{\sqrt{(n-1) \epsilon}} & \leq C_{h}, \\
\frac{\left|\phi\left(x, \hat{x}_{j}, \gamma_{j 1}(x), y_{2}, \ldots, y_{n}\right)-\phi\left(x, \hat{x}_{j}, \gamma_{j}(x)\right)\right|}{\sqrt{(n-1) \epsilon}} & \leq C_{h}
\end{aligned}
$$

for $x \in\left[t_{1}, t_{2}\right]$ and $y_{1} \in\left(\gamma_{j 1}(x)-\epsilon, \gamma_{j 1}(x)\right)$. Hence

$$
\begin{aligned}
& \int_{t_{1}}^{t_{2}} \int_{\gamma_{j 2}(x)-\epsilon}^{\gamma_{j 2}(x)} \ldots \int_{\gamma_{j n}(x)-\epsilon}^{\gamma_{j n}(x)} \frac{1}{2} b_{j 1}^{(1)}\left(\phi\left(x, \hat{x}_{j}, \gamma_{j 1}(x)-\epsilon, y_{2}, \ldots, y_{n}\right)\right. \\
& \left.\quad-\phi\left(x, \hat{x}_{j}, \gamma_{j 1}(x), y_{2}, \ldots, y_{n}\right)\right)\left(\phi\left(x, \hat{x}_{j}, \gamma_{j 1}(x)-\epsilon, y_{2}, \ldots, y_{n}\right)\right. \\
& \left.\quad+\phi\left(x, \hat{x}_{j}, \gamma_{j 1}(x), y_{2}, \ldots, y_{n}\right)-2 \phi\left(x, \hat{x}_{j}, \gamma_{j}(x)\right)\right) d y_{n} \ldots d y_{2} d x \\
& \leq\left|b_{j 1}^{(1)}\right| \sqrt{n-1} C_{h}^{2}\left(t_{2}-t_{1}\right) \epsilon^{n} .
\end{aligned}
$$


In a similar way, we can be found an estimate for each term on the right in (69).

Then putting together (69) and (71), dividing by $\epsilon^{n}$ and getting to the limit to $\epsilon \rightarrow 0$, it follows that there is $C=\left(C_{h}, b_{j 1}^{(1)}, \ldots, b_{j 1}^{(n)}\right)>0$ such that

$$
\phi\left(t_{2}, \hat{x}_{j}, \gamma_{j}\left(t_{2}\right)\right)-\phi\left(t_{1}, \hat{x}_{j}, \gamma_{j}\left(t_{1}\right)\right) \leq\left(\left\|w_{j}\right\|_{\mathcal{L}^{\infty}(\mathcal{O}, \mathbf{R})}+C\right)\left(t_{2}-t_{1}\right)
$$

for $t_{1}, t_{2} \in(-T, T)$ with $t_{1}<t_{2}$.

In a similar way, starting from (69) and using again (70) we can conclude

$$
\phi\left(t_{2}, \hat{x}_{j}, \gamma_{j}\left(t_{2}\right)\right)-\phi\left(t_{1}, \hat{x}_{j}, \gamma_{j}\left(t_{1}\right)\right) \geq-\left(\left\|w_{j}\right\|_{\mathcal{L}^{\infty}(\mathcal{O}, \mathbf{R})}+C\right)\left(t_{2}-t_{1}\right)
$$

for $t_{1}, t_{2} \in(-T, T)$ with $t_{1}<t_{2}$.

Hence combining (72) and (73), we get (67), i.e. $\phi$ is a Lipschitz map along any characteristic line $\gamma_{j}$ as desired.

Now we are able to show the proof of Theorem 7.2. In the case of Heisenberg groups the analogous proposition and proof of it are shown in [5], Lemma 5.4.

Proof. Fix $b \in \mathcal{O}$. According to Remark 2.16 (9), we would like to prove that there exists $\hat{C}>0$ such that

$$
\left|\phi\left(a^{\prime}\right)-\phi(a)\right| \leq \hat{C}\left\|\hat{\phi}(i(a))^{-1} i(a)^{-1} i\left(a^{\prime}\right) \hat{\phi}(i(a))\right\| \quad \text { for all } a, a^{\prime} \in \mathcal{U}(b, \delta)
$$

with $\delta>0$. Let $a=(x, y), a^{\prime}=\left(x^{\prime}, y^{\prime}\right)$ points of $\mathcal{O}$ be sufficiently close to $b$, and let $\bar{D}_{j}$ be the vector fields given by $\bar{D}_{j}=\left(x_{j}^{\prime}-x_{j}\right) D_{j}^{\phi}$ for $j \in\{2, \ldots, m\}$. We define

$$
\begin{aligned}
a_{1} & :=a, \\
a_{2} & :=\exp \left(\bar{D}_{2}\right)\left(a_{1}\right), \\
a_{3} & :=\exp \left(\bar{D}_{3}\right)\left(a_{2}\right), \\
& \vdots \\
a_{m} & :=\exp \left(\bar{D}_{m}\right)\left(a_{m-1}\right) .
\end{aligned}
$$

More precisely for $j \in\{2, \ldots, m\}$

$$
a_{j}=\left(x_{2}^{\prime}, \ldots, x_{j}^{\prime}, x_{j+1}, \ldots, x_{m}, y^{a_{j}}\right)
$$

with

$$
\begin{aligned}
y_{s}^{a_{j}}= & y_{s}+\sum_{l=2}^{j}\left(b_{l 1}^{(s)} \int_{0}^{x_{l}^{\prime}-x_{l}} \phi\left(\exp \left(r D_{l}^{\phi}\left(a_{l-1}\right)\right)\right) d r\right. \\
& \left.+\frac{1}{2}\left(x_{l}^{\prime}-x_{l}\right)\left(\sum_{h=2}^{l} x_{h}^{\prime} b_{l h}^{(s)}+\sum_{h=l+1}^{m} x_{h} b_{l h}^{(s)}\right)\right) \\
= & \left\{\begin{array}{l}
y_{s}^{a_{j-1}}+b_{j 1}^{(s)} \int_{0}^{x_{j}^{\prime}-x_{j}} \phi\left(\exp \left(r D_{j}^{\phi}\left(a_{j-1}\right)\right)\right) d r \\
+\frac{1}{2}\left(x_{j}^{\prime}-x_{j}\right)\left(\sum_{h=2}^{j} x_{h}^{\prime} b_{j h}^{(s)}+\sum_{h=j+1}^{m} x_{h} b_{j h}^{(s)}\right), \\
y_{s}^{a_{j-1}}+b_{j 1}^{(s)} \int_{0}^{x_{j}^{\prime}-x_{j}} \phi\left(\exp \left(r D_{j}^{\phi}\left(a_{j-1}\right)\right)\right) d r+\frac{1}{2}\left(x_{j}^{\prime}-x_{j}\right) \sum_{h=2}^{j} x_{h}^{\prime} b_{j h}^{(s)}, \quad \text { if } j=m,
\end{array}\right.
\end{aligned}
$$

for $s=1, \ldots, n$. By Lemma 7.3, $(-\delta, \delta) \ni r \mapsto \phi\left(\exp \left(r D_{j}^{\phi}\left(a_{j-1}\right)\right)\right)$ is Lipschitz for all $j=2, \ldots, m$ and so $a_{2}, \ldots, a_{m}$ are well defined if $a, a^{\prime} \in \mathcal{U}(b, \delta)$ for a sufficiently 
small $\delta>0$ (we recall that the global existence of integral curve of $D_{j}^{\phi}$ is not sure because the vector fields $D_{j}^{\phi}$ are only continuous).

We observe that

$$
\left|\phi\left(a^{\prime}\right)-\phi(a)\right| \leq\left|\phi\left(a^{\prime}\right)-\phi\left(a_{m}\right)\right|+\sum_{l=2}^{m}\left|\phi\left(a_{l}\right)-\phi\left(a_{l-1}\right)\right|
$$

and by Lemma 7.3 there is $C>0$ such that

$$
\sum_{l=2}^{m}\left|\phi\left(a_{l}\right)-\phi\left(a_{l-1}\right)\right| \leq \sum_{l=2}^{m} C\left|x_{l}^{\prime}-x_{l}\right|_{\mathbf{R}^{m-1}} \leq C\left\|\hat{\phi}(i(a))^{-1} i(a)^{-1} i\left(a^{\prime}\right) \hat{\phi}(i(a))\right\| .
$$

Hence in order to establish (74) we show that there exists $C_{1}>0$ such that

$$
\left|\phi\left(a^{\prime}\right)-\phi\left(a_{m}\right)\right| \leq C_{1}\left\|\hat{\phi}(i(a))^{-1} i(a)^{-1} i\left(a^{\prime}\right) \hat{\phi}(i(a))\right\| .
$$

Recalling that $a_{m}=\left(x^{\prime}, y^{a_{m}}\right)$ and using $\phi$ is locally $1 / 2$-Hölder continuous along the vertical components with Hölder's constant $C_{h}>0$, it follows

$$
\left|\phi\left(a^{\prime}\right)-\phi\left(a_{m}\right)\right| \leq C_{h}\left|y^{\prime}-y^{a_{m}}\right|_{\mathbf{R}^{n}}^{1 / 2}
$$

Moreover, arguing as in the proof of the implication 4 . $\Rightarrow 2$. in Theorem 5.7 in [11], we have

$$
\begin{aligned}
\left|y^{\prime}-y^{a_{m}}\right|_{\mathbf{R}^{n} \leq} \leq & \sum_{s=1}^{n} \mid y_{s}^{\prime}-y_{s}+\sum_{l=2}^{m}\left(b_{1 l}^{(s)} \int_{0}^{x_{l}^{\prime}-x_{l}} \phi\left(\exp \left(r D_{l}^{\phi}\left(a_{l-1}\right)\right)\right) d r+\right. \\
& \left.-\frac{1}{2}\left(x_{l}^{\prime}-x_{l}\right)\left(\sum_{i=2}^{l} x_{i}^{\prime} b_{l i}^{(s)}+\sum_{i=l+1}^{m} x_{i} b_{l i}^{(s)}\right)\right) \mid \\
\leq & \sum_{s=1}^{n}\left|y_{s}^{\prime}-y_{s}+\phi(a) \sum_{l=2}^{m}\left(x_{l}^{\prime}-x_{l}\right) b_{1 l}^{(s)}-\frac{1}{2}\left\langle\mathcal{B}^{(s)} x, x^{\prime}-x\right\rangle\right| \\
& +\sum_{s=1}^{n}\left|-\frac{1}{2} \sum_{l=2}^{m}\left(x_{l}^{\prime}-x_{l}\right)\left(\sum_{i=2}^{l} x_{i}^{\prime} b_{l i}^{(s)}+\sum_{i=l+1}^{m} x_{i} b_{l i}^{(s)}\right)+\frac{1}{2}\left\langle\mathcal{B}^{(s)} x, x^{\prime}-x\right\rangle\right| \\
& +\sum_{s=1}^{n}\left|-\phi(a) \sum_{l=2}^{m}\left(x_{l}^{\prime}-x_{l}\right) b_{1 l}^{(s)}+\sum_{l=2}^{m} b_{1 l}^{(s)} \int_{0}^{x_{l}^{\prime}-x_{l}} \phi\left(\exp \left(r D_{l}^{\phi}\left(a_{l-1}\right)\right)\right) d r\right| \\
\leq & c_{1}\left\|\hat{\phi}(i(a))^{-1} i(a)^{-1} i\left(a^{\prime}\right) \hat{\phi}(i(a))\right\|^{2}+\frac{1}{2} n\|\mathcal{B}\|_{\infty}\left|x^{\prime}-x\right|_{\mathbf{R}^{m-1}}^{2}+ \\
& +\sum_{s=1}^{n}\left|-\phi(a) \sum_{l=2}^{m}\left(x_{l}^{\prime}-x_{l}\right) b_{1 l}^{(s)}+\sum_{l=2}^{m} b_{1 l}^{(s)} \int_{0}^{x_{l}^{\prime}-x_{l}} \phi\left(\exp \left(r D_{l}^{\phi}\left(a_{l-1}\right)\right)\right) d r\right|
\end{aligned}
$$

where $c_{1}$ is given by $(11)$ and $\|\mathcal{B}\|_{\infty}=\max \left\{b_{i j}^{(s)} \mid i, j=1, \ldots, m, s=1, \ldots, n\right\}$. Note that we have used

$$
\begin{aligned}
& \frac{1}{2}\left\langle\mathcal{B}^{(s)} x, x^{\prime}-x\right\rangle-\frac{1}{2} \sum_{l=2}^{m}\left(x_{l}^{\prime}-x_{l}\right)\left(\sum_{i=2}^{l} x_{i}^{\prime} b_{l i}^{(s)}+\sum_{i=l+1}^{m} x_{i} b_{l i}^{(s)}\right) \\
& =-\frac{1}{2} \sum_{l=2}^{m}\left(x_{l}^{\prime}-x_{l}\right)\left(\sum_{i=2}^{l} x_{i}^{\prime} b_{l i}^{(s)}+\sum_{i=l+1}^{m} x_{i} b_{l i}^{(s)}-\sum_{i=2}^{m} x_{i} b_{l i}^{(s)}\right) \\
& \leq \frac{1}{2}\|\mathcal{B}\|_{\infty}\left|x^{\prime}-x\right|_{\mathbf{R}^{m-1}}^{2}
\end{aligned}
$$


Finally, the last term

$$
\begin{aligned}
& \sum_{s=1}^{n}\left|-\phi(a) \sum_{l=2}^{m}\left(x_{l}^{\prime}-x_{l}\right) b_{1 l}^{(s)}+\sum_{l=2}^{m} b_{1 l}^{(s)} \int_{0}^{x_{l}^{\prime}-x_{l}} \phi\left(\exp \left(r D_{l}^{\phi}\left(B_{l-1}\right)\right)\right) d r\right| \\
& \leq R_{1}\left(a, a^{\prime}\right)+R_{2}\left(a, a^{\prime}\right)
\end{aligned}
$$

where

$$
\begin{aligned}
& R_{1}\left(a, a^{\prime}\right):=\sum_{s=1}^{n} \sum_{l=2}^{m}\left|b_{1 l}^{(s)} \int_{0}^{x_{l}^{\prime}-x_{l}} \phi\left(\exp \left(r D_{l}^{\phi}\left(a_{l-1}\right)\right)\right) d r-b_{1 l}^{(s)} \phi\left(a_{l-1}\right)\left(x_{l}^{\prime}-x_{l}\right)\right|, \\
& R_{2}\left(a, a^{\prime}\right):=\sum_{s=1}^{n}\left|\sum_{l=2}^{m} b_{1 l}^{(s)}\left(x_{l}^{\prime}-x_{l}\right)\left(\phi\left(a_{l-1}\right)-\phi(a)\right)\right|
\end{aligned}
$$

We would like to show that there exist $C_{1}, C_{2}>0$ such that

$$
\begin{aligned}
& R_{1}\left(a, a^{\prime}\right) \leq C_{1}\left|x^{\prime}-x\right|_{\mathbf{R}^{m-1}}^{2}, \\
& R_{2}\left(a, a^{\prime}\right) \leq C_{2}\left|x^{\prime}-x\right|_{\mathbf{R}^{m-1}}^{2}
\end{aligned}
$$

for all $a, a^{\prime} \in \mathcal{U}(b, \delta)$ and consequently

$$
\left|y^{\prime}-y^{a_{m}}\right|_{\mathbf{R}^{n}} \leq c_{1}\left\|\hat{\phi}(i(a))^{-1} i(a)^{-1} i\left(a^{\prime}\right) \hat{\phi}(i(a))\right\|^{2}+\left(\frac{1}{2} n\|\mathcal{B}\|_{\infty}+C_{1}+C_{2}\right)\left|x^{\prime}-x\right|_{\mathbf{R}^{m-1}}^{2}
$$

Hence there is $C_{3}>0$ such that

$$
\left|y^{\prime}-y^{a_{m}}\right|_{\mathbf{R}^{n}}^{1 / 2} \leq C_{3}\left\|\hat{\phi}(i(a))^{-1} i(a)^{-1} i\left(a^{\prime}\right) \hat{\phi}(i(a))\right\|,
$$

i.e. (77) is true. We start to consider $R_{1}\left(a, a^{\prime}\right)$. Fix $l=2, \ldots, m$. For $t \in[-\delta, \delta]$ we define

$$
g_{l}(t):=\sum_{s=1}^{n} b_{1 l}^{(s)}\left(\int_{0}^{t} \phi\left(\exp \left(r D_{l}^{\phi}\right)\left(a_{l-1}\right)\right) d r-t \phi\left(a_{l-1}\right)\right) .
$$

Observe that

$$
b_{1 l}^{(s)} \int_{0}^{t}\left(\phi\left(\exp \left(r D_{l}^{\phi}\right)\left(a_{l-1}\right)\right)-\phi\left(a_{l-1}\right)\right) d r=O\left(t^{2}\right),
$$

and so there is $C_{l}>0$ such that

$$
\left|g_{l}(t)\right| \leq C_{l} t^{2}, \quad \forall t \in[-\delta, \delta] .
$$

Hence setting $t=x_{l}^{\prime}-x_{l}$ we get

$$
\left|g_{l}\left(x_{l}^{\prime}-x_{l}\right)\right| \leq C_{l}\left(x_{l}^{\prime}-x_{l}\right)^{2},
$$

and consequently (78) follows from

$$
\sum_{l=2}^{m}\left|g_{l}\left(x_{l}^{\prime}-x_{l}\right)\right| \leq \sum_{l=2}^{m} C_{l}\left(x_{l}^{\prime}-x_{l}\right)^{2} \leq C_{1}\left|x^{\prime}-x\right|_{\mathbf{R}^{m-1}}^{2}
$$


Now we consider $R_{2}\left(a, a^{\prime}\right)$. Observe that

$$
\begin{aligned}
& \sum_{s=1}^{n}\left|\sum_{l=2}^{m} b_{1 l}^{(s)}\left(x_{l}^{\prime}-x_{l}\right)\left(\phi\left(a_{l-1}\right)-\phi(a)\right)\right| \leq n\|\mathcal{B}\|_{\infty} \sum_{l=2}^{m}\left|x_{l}^{\prime}-x_{l}\right|\left|\phi\left(a_{l-1}\right)-\phi(a)\right| \\
& \leq n\|\mathcal{B}\|_{\infty} \sum_{l=2}^{m}\left|x_{l}^{\prime}-x_{l}\right|\left(\sum_{h=1}^{l-1}\left|\phi\left(a_{h}\right)-\phi\left(a_{h-1}\right)\right|\right) \quad\left(\text { where } a_{0}:=a\right) \\
& \leq n\|\mathcal{B}\|_{\infty} \sum_{l=2}^{m}\left|x_{l}^{\prime}-x_{l}\right|\left(\sum_{h=1}^{l-1}\left|\int_{0}^{1}\left(\bar{D}_{h} \phi\right)\left(\exp \left(r \bar{D}_{h}\left(a_{h-1}\right)\right)\right) d r\right|\right) \\
& \leq n\|\mathcal{B}\|_{\infty} \sum_{l=2}^{m}\left|x_{l}^{\prime}-x_{l}\right|\left(\sum_{h=1}^{l-1}\left|\left(x_{h}^{\prime}-x_{h}\right)\left(D_{h}^{\phi} \phi\left(a_{h-1}\right)+o(1)\right)\right|\right) \\
& \leq n\|\mathcal{B}\|_{\infty} C\left|x^{\prime}-x\right|_{\mathbf{R}^{m-1}}^{2} .
\end{aligned}
$$

Then (79) follows with $C_{2}:=n\|\mathcal{B}\|_{\infty} C$ and (80) is true. Finally, putting together (75), (76) and (77), (74) holds and the proof is complete.

\section{A characterization of G-regular hypersurfaces}

In this section we will prove a new characterization of G-regular hypersurfaces (see Definition 2.2). Here we consider the non linear first order system

$$
\left(D_{2}^{\phi} \phi, \ldots, D_{m}^{\phi} \phi\right)=w
$$

where $w: \mathcal{O} \rightarrow \mathbf{R}^{m-1}$ is a given continuous function and not just measurable as opposed to the previous sections.

In [11], we give some equivalent conditions for G-regular hypersurface inside the Carnot groups of step 2 (see Theorem 5.7 in [11]). In this section we give another one. More precisely, we show that if a continuous map $\phi: \mathcal{O} \subset \mathbf{R}^{m+n-1} \rightarrow \mathbf{R}$ is locally little 1/2-Hölder continuous along the vertical components, then $\phi$ is a distributional solution in an open set $\mathcal{O}$ of the non linear first order system (81) if and only if $\hat{\phi}: \hat{\mathcal{O}} \rightarrow \mathbf{V}$ is uniformly intrinsic differentiable in $\hat{\mathcal{O}}$ and consequently its graph is a G-regular hypersurface. The main equivalence result is contained in Theorem 8.2.

Its proof is similar to the one of [6]: firstly we have to be precise about the meaning of being a solution of (81). To this aim we recall a notion of generalized solutions of systems of this kind. These generalized solutions, denoted broad* solutions were introduced and studied for the system (81) inside Heisenberg groups in [3, 6]. For a more complete bibliography we refer to the bibliography in [3]. Then our strategy will be to prove that each continuous distributional solution of the system (81) is a broad* solution and vice versa (see Proposition 8.1).

The regularity results contained in [6] yield Hölder regularity properties for continuous distributional solutions of (81) and for broad* solutions of (81) in the context of Heisenberg groups. Hence in Heisenberg groups we can omit the little Hölder continuity of $\phi$ because each assumption implies it. We use this hypothesis in the proof of Proposition 8.1, but it is not clear if we can omit it.

Definition 8.1. Let $\mathcal{O} \subset \mathbf{R}^{m+n-1}$ be open and $w:=\left(w_{2}, \ldots, w_{m}\right): \mathcal{O} \rightarrow \mathbf{R}^{m-1}$ a continuous function. With the notations of Definition 3.1 we say that $\phi \in \mathbf{C}(\mathcal{O}, \mathbf{R})$ 
is a broad* ${ }^{*}$ solution in $\mathcal{O}$ of the system

$$
\left(D_{2}^{\phi} \phi, \ldots, D_{m}^{\phi} \phi\right)=w
$$

if for every $a_{0} \in \mathcal{O}$ there are $0<\delta_{2}<\delta_{1}$ and $m-1$ maps $\exp _{a_{0}}\left(\cdot D_{j}^{\phi}\right)(\cdot)$

$$
\exp _{a_{0}}\left(\cdot D_{j}^{\phi}\right)(\cdot):\left[-\delta_{2}, \delta_{2}\right] \times \mathcal{I}\left(a_{0}, \delta_{2}\right) \rightarrow \mathcal{I}\left(a_{0}, \delta_{1}\right)(t, a) \mapsto \exp _{a_{0}}\left(t D_{j}^{\phi}\right)(a)
$$

for $j=2, \ldots, m$, where $\mathcal{I}\left(a_{0}, \delta\right):=\mathcal{U}\left(a_{0}, \delta\right) \cap \mathbf{W}$ and $\mathcal{I}\left(a_{0}, \delta_{1}\right) \subset \mathcal{O}$. Moreover, these maps, called exponential maps of the vector fields $D_{2}^{\phi}, \ldots, D_{m}^{\phi}$, are required to have the following properties

$$
t \mapsto \gamma_{a}^{j}(t):=\exp _{a_{0}}\left(t D_{j}^{\phi}\right)(a) \in \mathbf{C}^{1}\left(\left[-\delta_{2}, \delta_{2}\right], \mathbf{R}^{m+n-1}\right)
$$

for all $a \in \mathcal{I}\left(a_{0}, \delta_{2}\right)$ and

$$
\begin{gathered}
\left\{\begin{array}{l}
\dot{\gamma}_{a}^{j}=D_{j}^{\phi} \circ \gamma_{a}^{j}, \\
\gamma_{a}^{j}(0)=a,
\end{array}\right. \\
\phi\left(\gamma_{a}^{j}(t)\right)-\phi\left(\gamma_{a}^{j}(0)\right)=\int_{0}^{t} w_{j}\left(\gamma_{a}^{j}(r)\right) d r, \quad \text { for } t \in\left[-\delta_{2}, \delta_{2}\right]
\end{gathered}
$$

once more for all $a \in \mathcal{I}\left(a_{0}, \delta_{2}\right)$.

Proposition 8.1. Let $\mathbf{G}:=\left(\mathbf{R}^{m+n}, \cdot, \delta_{\lambda}\right)$ be a Carnot group of step 2 and $\mathbf{V}$, $\mathbf{W}$ the complementary subgroups defined in (13). Let $\hat{\phi}: \hat{\mathcal{O}} \rightarrow \mathbf{V}$ be a continuous map, where $\hat{\mathcal{O}}$ is an open subset of $\mathbf{W}$ and $\phi: \mathcal{O} \rightarrow \mathbf{R}$ is the map associated to $\hat{\phi}$ as in (14). Then the following conditions are equivalent:

(1) $\phi$ is locally little $1 / 2$-Hölder continuous along the vertical components, i.e. that is $\phi \in \mathbf{C}(\mathcal{O}, \mathbf{R})$ and for all $\mathcal{O}^{\prime} \Subset \mathcal{O}$

$$
\lim _{r \rightarrow 0^{+}} \sup \left\{\frac{\left|\phi\left(x, y^{\prime}\right)-\phi(x, y)\right|}{\left|y^{\prime}-y\right|_{\mathbf{R}^{n}}^{1 / 2}}:(x, y),\left(x, y^{\prime}\right) \in \mathcal{O}^{\prime}, 0<\left|y^{\prime}-y\right|_{\mathbf{R}^{n}}<r\right\}=0,
$$

and there exists $w \in \mathbf{C}\left(\mathcal{O}, \mathbf{R}^{m-1}\right)$ such that $\phi$ is a broad* solution of

$$
\left(D_{2}^{\phi} \phi, \ldots, D_{m}^{\phi} \phi\right)=w, \quad \text { in } \mathcal{O}
$$

(2) $\phi$ is locally little $1 / 2$-Hölder continuous along the vertical components and there exists $w \in \mathbf{C}\left(\mathcal{O}, \mathbf{R}^{m-1}\right)$ such that $\phi$ is distributional solution of

$$
\left(D_{2}^{\phi} \phi, \ldots, D_{m}^{\phi} \phi\right)=w, \quad \text { in } \mathcal{O} \text {. }
$$

Proof. $(\mathbf{1}) \Longrightarrow(\mathbf{2})$. By Theorem 5.7 in [11], we know that there is a family of functions $\phi_{\epsilon} \in \mathbf{C}^{1}(\mathcal{O}, \mathbf{R})$ such that for all $\mathcal{O}^{\prime} \Subset \mathcal{O}$,

$$
\phi_{\epsilon} \rightarrow \phi \quad \text { and } \quad D_{j}^{\phi_{\epsilon}} \phi_{\epsilon} \rightarrow D_{j}^{\phi} \phi
$$

for $j=2, \ldots, m$ uniformly on $\mathcal{O}^{\prime}$ as $\epsilon \rightarrow 0^{+}$. Then for each $j=2, \ldots, m, \epsilon>0$ and $\zeta \in \mathbf{C}_{c}^{1}(\mathcal{O}, \mathbf{R})$

$$
\int_{\mathcal{O}} \phi_{\epsilon}\left(X_{j} \zeta+\phi_{\epsilon} \sum_{s=1}^{n} b_{j 1}^{(s)} Y_{s} \zeta\right) d \mathcal{L}^{m+n-1}=-\int_{\mathcal{O}} D_{j}^{\phi_{\epsilon}} \phi_{\epsilon} \zeta d \mathcal{L}^{m+n-1}
$$


Using (82) and getting to the limit for $\epsilon \rightarrow 0^{+}$we have that

$$
\int_{\mathcal{O}} \phi\left(X_{j} \zeta+\phi \sum_{s=1}^{n} b_{j 1}^{(s)} Y_{s} \zeta\right) d \mathcal{L}^{m+n-1}=-\int_{\mathcal{O}} D_{j}^{\phi} \phi \zeta d \mathcal{L}^{m+n-1} .
$$

Hence $\phi$ is distributional solution of $\left(D_{2}^{\phi} \phi, \ldots, D_{m}^{\phi} \phi\right)=w$ in $\mathcal{O}$.

$(2) \Longrightarrow(1)$. Let $a_{0} \in \mathcal{O}, \delta_{1}>0$ and $\mathcal{I}:=\mathcal{U}\left(a_{0}, \delta_{1}\right) \cap \mathbf{W}$ and $\mathcal{I}\left(a_{0}, \delta_{1}\right) \subset \mathcal{O}$.

Denote

$$
K:=\sup _{(x, y) \in \mathcal{I}} \sum_{l=2}^{m}\left|x_{l}\right|, \quad M:=\|\phi\|_{\mathcal{L}^{\infty}(\mathcal{I}, \mathbf{R})}, \quad \delta_{2}<\frac{\delta_{1}}{2+\frac{1}{2} K\|\mathcal{B}\|_{\infty}+M\|\mathcal{B}\|_{\infty}},
$$

where $\|\mathcal{B}\|_{\infty}=\max \left\{b_{j l}^{(s)} \mid l, j=1, \ldots, m, s=1, \ldots, n\right\}$. Peano's Theorem yields that for all $a=\left(x_{j}, \hat{x}_{j}, y\right) \in \overline{\mathcal{I}\left(a_{0}, \delta_{2}\right)}$ there exists a $\mathbf{C}^{1}$ function $\left(\gamma_{j 1}, \ldots, \gamma_{j n}\right):\left[-\delta_{2}, \delta_{2}\right] \rightarrow$ $\mathbf{R}^{n}$ such that

$$
\gamma_{a}^{j}(t)=\left(x_{j}+t, \hat{x}_{j}, \gamma_{j 1}(t), \ldots, \gamma_{j n}(t)\right) \in \mathcal{I} \quad \text { for } t \in\left[-\delta_{2}, \delta_{2}\right],
$$

and $\gamma_{j s}(t)$ is a solution of the Cauchy problem

$$
\left\{\begin{array}{l}
\dot{\gamma}_{j s}(t)=\frac{1}{2} \sum_{\substack{l=2 \\
l \neq j}}^{m} x_{l} b_{j l}^{(s)}+b_{j 1}^{(s)} \phi\left(\gamma_{a}^{j}(t)\right), \text { for } t \in\left[-\delta_{2}, \delta_{2}\right] \\
\gamma_{j s}(0)=y_{s}
\end{array}\right.
$$

for $s=1, \ldots, n$. It is clear that if $b_{j 1}^{(s)}=0$, then $\gamma_{j s}(t)=y_{s}+\frac{1}{2} t \sum_{\substack{l=2 \\ l \neq j}}^{m} x_{l} b_{j l}^{(s)}$ for $t \in\left[-\delta_{2}, \delta_{2}\right]$. On the other hand, if $b_{j 1}^{(s)} \neq 0$, then the map $\phi\left(\gamma_{a}^{j}(\cdot)\right)$ satisfies the following ODE

$$
\frac{d}{d t}\left(b_{j 1}^{(s)} \phi\left(\gamma_{a}^{j}(t)\right)\right)=b_{j 1}^{(s)} w_{j}\left(\gamma_{a}^{j}(t)\right)
$$

with $t \in[-\delta, \delta]$ for some $\delta>0$. Indeed, we can repeat verbatim the proof of Lemma 7.3 and so we obtain (69) with $b_{j 1}^{(s)} \neq 0$. Moreover because $\phi$ is locally little $1 / 2$-Hölder continuous along the vertical components, upon dividing (69) by $\epsilon^{n}$ and getting to the limit to $\epsilon \rightarrow 0$, we have that

$$
\phi\left(\gamma_{a}^{j}\left(t_{2}\right)\right)-\phi\left(\gamma_{a}^{j}\left(t_{1}\right)\right)-\int_{t_{1}}^{t_{2}} w_{j}\left(\gamma_{a}^{j}(t)\right) d t=0
$$

for $t_{1}, t_{2} \in[-\delta, \delta]$ with $\delta>0$ and $t_{1}<t_{2}$.

In particular, $\phi\left(\gamma_{a}^{j}(\cdot)\right)$ and $\dot{\gamma}_{j s}(\cdot)$ are $\mathbf{C}^{1}\left(\left[-\delta_{3}, \delta_{3}\right], \mathbf{R}\right)$ for $s=1, \ldots, n$ where $\delta_{3}:=$ $\min \left\{\delta, \delta_{2}\right\}$. Therefore the curve $\gamma_{a}^{j}:\left[-\delta_{3}, \delta_{3}\right] \rightarrow \mathcal{I}$ satisfies the conditions of the Definition 8.1 for each $a \in \mathcal{I}\left(a_{0}, \delta_{3}\right):=\mathcal{U}\left(a_{0}, \delta_{3}\right) \cap \mathbf{W}$ and $\mathcal{I}\left(a_{0}, \delta_{3}\right) \subset \mathcal{O}$.

Then, for each $j=2, \ldots, m$, $\exp _{a_{0}}\left(\cdot D_{j}^{\phi}\right)(\cdot):\left[-\delta_{3}, \delta_{3}\right] \times \mathcal{I}\left(a_{0}, \delta_{3}\right) \rightarrow \mathcal{I}$ defined as $\exp _{a_{0}}\left(t D_{j}^{\phi}\right)(a):=\gamma_{a}^{j}(t)$ is a family of exponential maps at $a_{0}$ which we were looking for. This completes the proof of the implication $(2) \Longrightarrow(1)$.

Thanks to Proposition 8.1 and Theorem 5.7 in [11], we obtain an important result, i.e. Theorem 8.2, which is the counterpart of Theorem 1.2 and Theorem 1.3 in [3] in the context of Heisenberg groups.

Theorem 8.2. Let $\mathbf{G}:=\left(\mathbf{R}^{m+n}, \cdot, \delta_{\lambda}\right)$ be a Carnot group of step 2 and $\mathbf{V}, \mathbf{W}$ the complementary subgroups defined in (13). Let $\hat{\phi}: \hat{\mathcal{O}} \rightarrow \mathbf{V}$ be a continuous map, where $\hat{\mathcal{O}}$ is an open subset of $\mathbf{W}$ and $\phi: \mathcal{O} \rightarrow \mathbf{R}$ is the map associated to $\hat{\phi}$ as in (14). Then the following conditions are equivalent: 
(1) graph $(\hat{\phi})$ is a G-regular hypersurface and for all $a \in \operatorname{graph}(\hat{\phi})$ there is $r=$ $r(a)>0$ and $\left.f \in \mathbf{C}_{\mathbf{G}}^{1}(\mathcal{U}(a, r)), \mathbf{R}\right)$ with $X_{1} f>0$ such that

$$
\operatorname{graph}(\hat{\phi}) \cap \mathcal{U}(a, r)=\{p: f(p)=0\},
$$

(2) $\hat{\phi}$ is u.i.d. in $\hat{\mathcal{O}}$,

(3) $D_{j}^{\phi} \phi$ interpreted in distributional sense is a continuous function in $\mathcal{O}$ and for $0<\epsilon<1$ there is a family of functions $\phi_{\epsilon} \in \mathbf{C}^{1}(\mathcal{O}, \mathbf{R})$ such that for all $\mathcal{O}^{\prime} \Subset \mathcal{O}$,

$$
\phi_{\epsilon} \rightarrow \phi \quad \text { and } \quad D_{j}^{\phi_{\epsilon}} \phi_{\epsilon} \rightarrow D_{j}^{\phi} \phi
$$

for $j=2, \ldots, m$, uniformly on $\mathcal{O}^{\prime}$ as $\epsilon \rightarrow 0^{+}$,

(4) $\phi$ is locally little $1 / 2$-Hölder continuous along the vertical components and there exists $w \in \mathbf{C}\left(\mathcal{O}, \mathbf{R}^{m-1}\right)$ such that $\phi$ is a broad* solution of

$$
\left(D_{2}^{\phi} \phi, \ldots, D_{m}^{\phi} \phi\right)=w, \quad \text { in } \mathcal{O}
$$

(5) $\phi$ is locally little $1 / 2$-Hölder continuous along the vertical components and there exists $w \in \mathbf{C}\left(\mathcal{O}, \mathbf{R}^{m-1}\right)$ such that $\phi$ is distributional solution of

$$
\left(D_{2}^{\phi} \phi, \ldots, D_{m}^{\phi} \phi\right)=w, \quad \text { in } \mathcal{O} \text {. }
$$

Remark 8.3. We recall that in Theorem 5.7 in [11], we consider a locally little $1 / 2$-Hölder continuous map but in the proof we just use that $\phi$ is locally little $1 / 2$ Hölder continuous along the vertical components.

\section{References}

[1] Ambrosio, L.: Some fine properties of sets of finite perimeter in Ahlfors regular metric measure spaces. - Adv. Math. 159, 2001, 51-67.

[2] Ambrosio, L., and B. Kirchheim: Rectifiable sets in metric and Banach spaces. - Math. Ann. 318:3, 2000, 527-555.

[3] Ambrosio, L., F. Serra Cassano, and D. Vittone: Intrinsic regular hypersurfaces in Heisenberg groups. - J. Geom. Anal. 16, 2006, 187-232.

[4] Arena, G., and R. Serapioni: Intrinsic regular submanifolds in Heisenberg groups are differentiable graphs. - Calc. Var. Partial Differential Equations 35:4, 2009, 517-536.

[5] Bigolin, F., L. Caravenna, and F. Serra Cassano: Intrinsic Lipschitz graphs in Heisenberg groups and continuous solutions of a balance equation. - Ann. Inst. H. Poincaré Anal. Non Linéaire, 2014.

[6] Bigolin, F., and F. Serra Cassano: Intrinsic regular graphs in Heisenberg groups vs. weak solutions of non linear first-order PDEs. - Adv. Calc. Var. 3, 2010, 69-97.

[7] Bonfiglioli, A., E. Lanconelli, and F. Uguzzoni: Stratified Lie groups and potential theory for their sub-Laplacians. - Springer Monogr. Math., Springer-Verlag, Berlin, Heidelberg, New York, 2007.

[8] Capogna, L., D. Danielli, and N. Garofalo: The geometric Sobolev embedding for vector fields and the isoperimetric inequality. - Comm. Anal. Geom. 12, 1994, 203-215.

[9] Citti, G., M. Manfredini, A. Pinamonti, and F. Serra Cassano: Smooth approximation for the intrinsic Lipschitz functions in the Heisenberg group. - Calc. Var. Partial Differentail Equations 49, 2014, 1279-1308.

[10] Dafermos, C. M.: Continuous solutions for balance laws. - Ric. Mat. 55, 2006, 79-91.

[11] Di Donato, D.: Intrinsic differentiability and intrinsic regular surfaces in Carnot groups. Preprint, arXiv:1811.05457. 
[12] Di Donato, D.: Intrinsic differentiability and intrinsic regular surfaces in Carnot groups. PhD Thesis, University of Trento, 2017.

[13] Evans, L. C., and R. F. Gariepy: Measure theory and fine properties of functions. - CRC Press, Boca Raton, 1992.

[14] Federer, H.: Geometric measure theory. - Springer, 1969.

[15] Folland, G. B., and E. M. Stein: Hardy spaces on homogeneous groups. - Princeton Univ. Press, 1982.

[16] Franchi, B., M. Marchi, and R. Serapioni: Differentiability and approximate differentiability for intrinsic Lipschitz functions in Carnot groups and a Rademarcher theorem. - Anal. Geom. Metr. Spaces 2, 2014, 258-281.

[17] Franchi, B., and R. Serapioni: Intrinsic Lipschitz graphs within Carnot groups. - J. Geom. Anal. 26:3, 2016, 1946-1994.

[18] Franchi, B., R. Serapioni, and F. Serra Cassano: Rectifiability and perimeter in the Heisenberg group. - Math. Ann. 321, 2001, 479-531.

[19] Franchi, B., R. Serapioni, and F. Serra Cassano: Regular hypersurfaces, intrinsic perimeter and implicit function theorem in Carnot groups. - Comm. Anal. Geom. 11:5, 2003, 909-944.

[20] Franchi, B., R. Serapioni, and F. Serra Cassano: On the Structure of finite perimeter sets in step 2 Carnot groups. - J. Geom. Anal. 13:3, 2003, 421-466.

[21] Franchi, B., R. Serapioni, and F. Serra Cassano: Intrinsic Lipschitz graphs in Heisenberg groups. - J. Nonlinear Convex Anal. 7:3, 2006, 423-441.

[22] Franchi, B., R. Serapioni, and F. Serra Cassano: Regular submanifolds, graphs and area formula in Heisenberg groups. - Adv. Math. 211:1, 2007, 152-203.

[23] Franchi, B., R. Serapioni, and F. Serra Cassano: Area formula for centered Hausdorff measures in mketric spaces. - Nonlinear Anal., 2015, 218-233.

[24] Garofalo, N., and D. M. Nhieu: Isoperimetric and Sobolev inequalities for CarnotCarathéodory spaces and the existence of minimal surfaces. - Comm. Pure Appl. Math. 49, 1996, 1081-1144.

[25] Gromov, M.: Carnot-Carathéodory spaces seen from within. - In: Sub Riemannian Geometry, Progr. Math. 144, ed. by A. Bellaïche and J. Risler, Birkhauser Verlag, Basel, 1996.

[26] Kirchheim, B., and F. Serra Cassano: Rectifiability and parametrization of intrinsic regular surfaces in the Heisenberg group. - Ann. Scuola Norm. Sup. Pisa Cl. Sci. (5) III, 2004, 871-896.

[27] Korte, R., and P. LAHTi: Relative isoperimetric inequalities and sufficient conditions for finite perimeter on metric spaces. - Ann. Inst. H. Poincaré Anal. Non Linéaire 31:1, 2014, $129-154$.

[28] Kozhevnikov, A.: Propriétés métriques des ensembles de niveau des applications différentiables sur les groupes de Carnot. - PhD Thesis, Université Paris-Sud, 2015.

[29] Le Donne, E.: A primer on Carnot groups: homogenous groups, Carnot-Carathéodory spaces, and regularity of their isometries. - Anal. Geom. Metr. Spaces 5, 2017, 116-137.

[30] Magnani, V.: Towards differential calculus in stratified groups. - J. Aust. Math. Soc. 95:1, 2013, 76-128.

[31] Magnani, V.: A new differentiation, shape of the unit ball and perimeter measure. - Indiana Univ. Math. J. 66:1, 2017, 183-204.

[32] Mattila, P.: Geometry of sets and measures in Euclidean spaces. - Cambridge Univ. Press, Cambridge, 1995.

[33] Mitchell, J.: On Carnot-Carathéodory metrics. - J. Differential Geom. 21, 1985, 35-45. 
[34] Monti, R., and D. Vittone: Sets with finite H-perimeter and controlled normal. - Math. Z. 270:1-2, 2012, 351-367.

[35] Pansu, P.: Métriques de Carnot-Carathéodory et quasiisométries des espaces symétriques de rang un. - Ann. of Math. (2) 129, 1989, 1-60.

[36] Serapioni, R.: Intrinsic difference quotients. - In: Harmonic Analysis, Partial Differential Equations and Applications, Birkhäuser, 2017, 165-192.

[37] Serra Cassano, F.: Some topics of geometric measure theory in Carnot groups. - In: Geometry, Analysis and Dynamics on sub-Riemannian Manifolds, Vol. I, Eur. Math. Soc., 2016, $1-121$.

[38] Serra Cassano, F., and D. Vittone: Graphs of bounded variation, existence and local boundedness of non-parametric minimal surfaces in the Heisenberg group. - Adv. Calc. Var. 7:4, 2014, 409-492.

[39] Visintin, A.: Strong convergence results related to strict convexity. - Comm. Partial Differential Equations 9, 1984, 439-466.

[40] Vittone, D.: Submanifolds in Carnot groups. - PhD Thesis, Scuola Normale Superiore, Edizioni della Normale, Pisa, 2008.

Received 6 March 2019 • Revised received 16 November 2019 • Accepted 23 December 2019 